\title{
Metalogical Decorations of Logical Diagrams
}

\author{
Lorenz Demey and Hans Smessaert
}

\begin{abstract}
In recent years, a number of authors have started studying Aristotelian diagrams containing metalogical notions, such as tautology, contradiction, satisfiability, contingency, strong and weak interpretations of (sub)contrariety, etc. The present paper is a contribution to this line of research, and its main aims are both to extend and to deepen our understanding of metalogical diagrams. As for extensions, we not only study several metalogical decorations of larger and less widely known Aristotelian diagrams, but also consider metalogical decorations of another type of logical diagrams, viz. duality diagrams. At a more fundamental level, we present a unifying perspective which sheds new light on the connections between new and existing metalogical diagrams, as well as between object- and metalogical diagrams. Overall, the paper studies two types of logical diagrams (viz. Aristotelian and duality diagrams) and four kinds of metalogical decorations (viz. those based on the opposition, implication, Aristotelian and duality relations).
\end{abstract}

Mathematics Subject Classification (2010). Primary 03A10, 03B45; Secondary 03B65, 03B80.

Keywords. Aristotelian diagram, duality diagram, metalogic, opposition geometry, implication geometry, contrariety.

\section{Introduction}

The Aristotelian square of oppositions is a diagram that displays four statements or notions, and certain logical relations holding between them. It has a very rich tradition, going back - together with the discipline of logic itself- to the works of Aristotle. Over the centuries, authors such as Avicenna, William of Sherwood, John Buridan, Boole and Frege have studied the square and other, larger Aristotelian diagrams [15, 16, 40, 48, 63, 67]. Since the beginning of the 21st century, logicians have started studying Aristotelian diagrams

Parts of this paper were presented at CLMPS 15 (Helsinki). We would like to thank the audience of that talk, as well as Jean-Yves Béziau, Alessio Moretti, Frédéric Sart, Fabien Schang, Margaux Smets and three anonymous reviewers for their valuable feedback. The first author holds a postdoctoral scholarship of the Research Foundation - Flanders (FWO). 
in a more systematic and mathematically precise way, which has led to significant theoretical advances, e.g. concerning the classification of the various types of Aristotelian diagrams [1, 59, 79, 81].

The overwhelming majority of Aristotelian diagrams that have been studied so far have object-level decorations: they visualize the Aristotelian relations holding between formulas coming from (the object language of) some given logical system (for example, propositional logic [55], modal logic [35], temporal logic [68], epistemic logic [50], deontic logic [56] and dynamic logic [22]), or between the natural language expressions of some given lexical field (for example, color terms [44], singular expressions [75] and subjective quantification [80]). In the last two or three years, however, authors such as Béziau and Seuren have also started investigating Aristotelian diagrams that are decorated with meta-level notions such as tautology, contradiction, satisfiability, contingency, strong and weak interpretations of (sub)contrariety, etc. $[4,5,33,72]$. As will be explained later in this paper, this transition from object- to metalogical decorations of Aristotelian diagrams is quite important, since it sheds some interesting new light on the ways in which the Aristotelian relations are usually defined. It should also be emphasized that these metalogical diagrams are not only important from a strictly theoretical perspective, but are also relevant in more practically oriented contexts. For example, it has been argued that the metalogical square can be fruitfully used in teaching metalogic to certain groups of students [24].

In sum, then, metalogical diagrams are becoming an active topic of investigation in contemporary philosophical logic. The present paper is a contribution to this line of research, and its main aims are both to extend and to deepen our understanding of metalogical diagrams. We will now explain in some more detail how each of these aims will be achieved.

The paper offers extensions vis-à-vis previous work in at least three ways. First of all, we will study much larger metalogical diagrams: while previous work has focused on metalogical squares and hexagons, we will also study metalogical decorations for larger Aristotelian diagrams, such as octagons, dodecagons, and even three-dimensional polyhedra such as the rhombic dodecahedron. Secondly, we will also study metalogical decorations for less widely known families of Aristotelian diagrams. This applies not only to the larger diagrams mentioned in the first point (obviously, a rhombic dodecahedron is less familiar to most people than an ordinary square of oppositions), but also to the smaller diagrams, such as hexagons: previous work has focused exclusively on a single family of hexagons, viz. the Jacoby-SesmatBlanché hexagons, but in this paper we will also consider other, less widely known families of hexagons, such as the 'Sherwood-Czezowski' hexagons, the 'unconnected-4' hexagons and the 'unconnected-8' hexagons. Thirdly, we will show that metalogical decorations can be given not only for Aristotelian diagrams, but also for other types of logical diagrams, such as Hasse diagrams and, most importantly, duality diagrams. 
There are also various ways in which the paper will deepen our understanding of metalogical diagrams. First of all, we will offer a more precise account of the relationship between object- and metalogical decorations of Aristotelian diagrams. Secondly, we will discuss the connections between the new metalogical diagrams presented in this paper and those that were previously studied. For example, we will show that the metalogical squares and hexagons studied by Béziau [4, 5], Diaconescu [33] and Seuren [72] can be seen as subdiagrams or specific instances of the diagrams presented here. Thirdly, because of these connections, the paper also offers a unifying perspective on metalogical diagrams. After all, if two seemingly unrelated metalogical diagrams can both be seen as specific instances of a single diagram, then they turn out to be intimately related to each other.

The paper is organized as follows. Sections 2 and 3 mainly prepare the ground for the analysis that will be presented in the later sections. Section 2 provides a series of increasingly more abstract ways of defining the Aristotelian relations, and discusses which of these definitions are best able to accommodate meta- as well as object-logical decorations of Aristotelian diagrams. Next, Section 3 provides some background on other types of logical relations, viz. the opposition, implication and duality relations. Sections 4-8 contain the core results of this paper: we present and study various metalogical decorations of various kinds of logical diagrams. Section 4 studies several Aristotelian diagrams that are decorated with the opposition relations, and discusses their connections with earlier work on metalogical decorations of Aristotelian diagrams. Next, Section 5 studies Aristotelian diagrams for the implication relations, and explores their connections with Aristotelian diagrams for the opposition relations (which were studied in Section 4) and for abstract ordering relations. Section 6 concludes our study of metalogical decorations for Aristotelian diagrams, by exploring some Aristotelian diagrams for the Aristotelian relations themselves, and for the duality relations. After this, we turn from metalogical Aristotelian diagrams to metalogical duality diagrams. In Section 7, we first study duality diagrams for the opposition and implication relations. Section 8 then explores some duality diagrams for the Aristotelian relations, and for the duality relations themselves. Finally, Section 9 summarizes the results that have been obtained in this paper, and highlights some general themes that we have touched upon along the way.

\section{Defining the Aristotelian Relations}

Before we start constructing new metalogical diagrams, it is important to clearly understand why a single type of diagrams - viz. Aristotelian diagramscan be used for meta- as well as object-logical decorations. In other words, how is it possible for the Aristotelian relations to hold between metalogical statements as well as between object-logical formulas? The exact way in which the Aristotelian relations are defined turns out to be highly relevant in answering this question. In this section, we will introduce a series of 
increasingly more abstract ways of defining the Aristotelian relations, and discuss to what extent these definitions are able to accommodate metalogical statements as well as object-logical formulas.

The oldest definition dates back to Aristotle himself, and has been used throughout the history of philophical logic [62]. In contemporary work on Aristotelian diagrams, too, it is by far the most widely used definition [8, 9]. The formulation is entirely informal, and looks as follows:

Definition 2.1. Two statements $\varphi$ and $\psi$ are said to be

\begin{tabular}{|c|c|c|}
\hline contradictory & iff & $\begin{array}{l}\varphi \text { and } \psi \text { cannot be true together } \\
\varphi \text { and } \psi \text { cannot be false together, }\end{array}$ \\
\hline contrary & iff & $\begin{array}{l}\varphi \text { and } \psi \text { cannot be true together } \\
\varphi \text { and } \psi \text { can be false together, }\end{array}$ \\
\hline subcontrary & iff & $\begin{array}{l}\varphi \text { and } \psi \text { can be true together } \\
\varphi \text { and } \psi \text { cannot be false together, }\end{array}$ \\
\hline in subalternation & iff & $\begin{array}{l}\varphi \text { entails } \psi \\
\psi \text { does not entail } \varphi\end{array}$ \\
\hline
\end{tabular}

Because of its informal nature, this definition can apply both to objectand to metalogical statements. To illustrate this, consider the ambiguity of the word 'true' in the definition of contrariety: this word can stand for 'truth in a model' (in case two object-logical formulas are said to be contrary), or for 'absolute, informal truth' (in case two metalogical statements are said to be contrary). A problem with Definition 2.1 is that it makes the Aristotelian relations entirely insensitive to the 'background logic'. Which Aristotelian relation holds between two (object-logical) formulas partially depends on the logical system that is being assumed. The well-known issue of 'existential import' can be seen as an illustration of this problem: in classical syllogistics, there is a subalternation from $\forall x(S x \rightarrow P x)$ to $\exists x(S x \wedge P x)$, but in contemporary predicate logic, these formulas stand in no Aristotelian relation at all [30, Section 4]. In order to deal with this problem, a new and more precise way of defining the Aristotelian relations has been proposed [75, 79, 80]:

Definition 2.2. Let $S$ be a logical system, which is assumed to have Boolean operators and a model-theoretic semantics $\mid=$. Two formulas $\varphi, \psi \in \mathcal{L}_{\mathrm{S}}$ are said to be

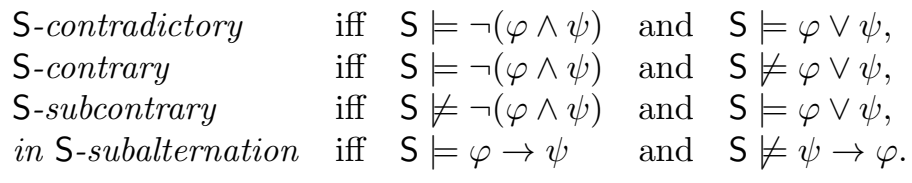

First of all, note that this definition stays very close to the original, more informal Definition 2.1. The condition that $\varphi$ and $\psi$ cannot be true together is formalized as $\mathrm{S} \models \neg(\varphi \wedge \psi)$, i.e. $\mathrm{S}$ has no models in which $\varphi$ and $\psi$ are simultaneously true; similarly, the condition that $\varphi$ and $\psi$ cannot be false together is formalized as $\mathrm{S} \models \varphi \vee \psi$, or equivalently, $\mathrm{S} \models \neg(\neg \varphi \wedge \neg \psi)$, i.e. $\mathrm{S}$ has no models in which $\varphi$ and $\psi$ are simultaneously false. Furthermore, note 
that unlike Definition 2.1, Definition 2.2 is capable of dealing with the logicsensitivity of the Aristotelian relations; for example, it is now possible to say that two formulas are $S_{1}$-contrary, but $S_{2}$-contradictory, for logical systems $S_{1}$ and $S_{2}$. The downside of explicitly specifying the logical system $S$, however, is that Definition 2.2 only applies to object-logical formulas: conditions such as $\mathrm{S} \models \neg(\varphi \wedge \psi)$ and $\mathrm{S} \models \varphi \rightarrow \psi$ are only meaningful for formulas $\varphi$ and $\psi$ from the object language $\mathcal{L}_{\mathrm{S}}$.

A key insight of Definition 2.2 is that the Aristotelian relations are fully determined by the Boolean structure of the logical system S. This suggests a third and final way of defining these relations, which abstracts away from the concrete details of $\mathbf{S}$, and only focuses on its Boolean structure:

Definition 2.3. Let $\mathbb{B}=\left\langle B, \wedge_{\mathbb{B}}, \vee_{\mathbb{B}}, \neg_{\mathbb{B}}, \top_{\mathbb{B}}, \perp_{\mathbb{B}}\right\rangle$ be a Boolean algebra. Two elements $x, y \in B$ are said to be

$\begin{array}{lllll}\mathbb{B} \text {-contradictory } & \text { iff } & x \wedge_{\mathbb{B}} y=\perp_{\mathbb{B}} & \text { and } & x \vee_{\mathbb{B}} y=\top_{\mathbb{B}}, \\ \mathbb{B} \text {-contrary } & \text { iff } & x \wedge_{\mathbb{B}} y=\perp_{\mathbb{B}} & \text { and } & x \vee_{\mathbb{B}} y \neq \top_{\mathbb{B}}, \\ \mathbb{B} \text {-subcontrary } & \text { iff } & x \wedge_{\mathbb{B}} y \neq \perp_{\mathbb{B}} & \text { and } & x \vee_{\mathbb{B}} y=\top_{\mathbb{B}}, \\ \text { in } \mathbb{B} \text {-subalternation } & \text { iff } & x \wedge_{\mathbb{B}} y=x & \text { and } & x \wedge_{\mathbb{B}} y \neq y .\end{array}$

Because of its level of abstraction, this definition is fully general. First, note that it subsumes Definition 2.2 as a special case: if $\mathrm{S}$ is a logical system as specified in Definition 2.2 (i.e. having Boolean connectives), then its Lindenbaum-Tarski algebra $\mathbb{B}(S):=\left\{[\varphi] \mid \varphi \in \mathcal{L}_{\mathrm{S}}\right\}$ is a Boolean algebra, and in the case of contrariety, for example, we have for all $\varphi, \psi \in \mathcal{L}_{\mathrm{S}}{ }^{1}$

$$
\begin{aligned}
\varphi \text { and } \psi \text { are S-contrary } & \text { iff } S \models \neg(\varphi \wedge \psi) \text { and } \mathrm{S} \not \nu \vee \psi \\
& \text { iff }[\varphi] \wedge[\psi]=\perp \text { and }[\varphi] \vee[\psi] \neq \top \\
& \text { iff }[\varphi] \text { and }[\psi] \text { are } \mathbb{B}(S) \text {-contrary. }
\end{aligned}
$$

Another set of special cases of Definition 2.3 arises if we take the Boolean algebra $\mathbb{B}$ to be the powerset $\wp(X)$ of some set $X$. In this case, two sets $X_{1}, X_{2} \subseteq X$ are $\wp(X)$-contrary iff $X_{1} \cap X_{2}=\emptyset$ and $X_{1} \cup X_{2} \neq X$-in other words, iff $X_{1}$ and $X_{2}$ are disjoint but not exhaustive. If we take $X$ to be the Lindenbaum-Tarski algebra $\mathbb{B}(\mathrm{S})$ of some (non-trivial) logical system $\mathrm{S}$, we see how Aristotelian relations can hold between metalogical statements. For example, if we consider the sets $X_{1}:=\{\top\}, X_{2}:=\{\perp\} \subseteq \mathbb{B}(\mathrm{S})$, we see that $X_{1}$ and $X_{2}$ are $\wp(\mathbb{B}(\mathrm{S}))$-contrary, which means exactly that the metalogical properties of being an S-tautology and being an S-contradiction - or equivalently, the metalogical statements " $\varphi$ is an S-tautology" and " $\varphi$ is an S-contradiction" - are contrary to each other. Finally, it will also be interesting to consider the case $X:=\mathbb{B}(\mathrm{S}) \times \mathbb{B}(\mathrm{S})$, i.e. $X$ is the set of all pairs of (equivalence classes of) formulas of the logical system $S$. Now we can define $X_{1}:=\{([\varphi],[\psi]) \mid \varphi$ and $\psi$ are S-contrary $\}$ and $X_{2}:=\{([\varphi],[\psi]) \mid$

\footnotetext{
${ }^{1}$ We make use here of the fact that the Aristotelian relations are only defined up to logical equivalence, i.e. if $\varphi \equiv_{\mathrm{S}} \varphi^{\prime}$ and $\psi \equiv_{\mathrm{S}} \psi^{\prime}$, then $R(\varphi, \psi)$ iff $R\left(\varphi^{\prime}, \psi^{\prime}\right)$, for all Aristotelian relations $R$. This can easily be derived from Definition 2.2 .
} 
$\varphi$ and $\psi$ are S-subcontrary $\}$, and see that $X_{1}$ and $X_{2}$ are $\wp(\mathbb{B}(\mathrm{S}) \times \mathbb{B}(\mathrm{S}))$ contrary, which means exactly that the metalogical relations of being $S$ contrary and being S-subcontrary - or equivalently, the metalogical statements " $\varphi$ and $\psi$ are S-contrary" and " $\varphi$ and $\psi$ are S-subcontrary" - are themselves contrary to each other.

Definition 2.3 thus explains why the Aristotelian relations can hold between meta- as well as object-logical statements: these relations are defined in a Boolean algebra, and this Boolean algebra can be situated at the objectlogical level - in the the case of $\mathbb{B}(\mathrm{S})$ - , or at the metalogical level - in the cases of $\wp(\mathbb{B}(S))$ and $\wp(\mathbb{B}(S) \times \mathbb{B}(S))$. In Sections 4,5 and 6 we will study metalogical decorations of Aristotelian diagrams that are based on both of the latter cases. We finish this section by summarizing the dialectical progression that has been developed:

- Definition 2.1 accommodates object- and metalogical statements, but fails to handle logic-sensitivity (at the object-level).

- Definition 2.2 is able to handle logic-sensitivity (at the object-level), but can only accommodate object-logical formulas.

- Definition 2.3 accommodates object- and metalogical statements, and is able to handle logic-sensitivity (at the object- and meta-level).

\section{Opposition, Implication and Duality}

In this section, we will introduce some other types of logical relations that are closely related to the Aristotelian relations, and that will play an important role later in the paper. Subsection 3.1 discusses the opposition and implication relations, which will be studied extensively in Sections 4,5 and 7 . Next, Subsection 3.2 deals with the duality relations, which will figure prominently in Sections 6 and 8.

\subsection{The Opposition and Implication Relations}

In [79] it is pointed out that the set of Aristotelian relations has a number of problematic, or at least peculiar, properties; for example, there is a clear conceptual asymmetry between the relations of contradiction, contrariety and subcontrariety on the one hand, and the relation of subalternation on the other. Furthermore, it is argued that these properties can best be explained by viewing the Aristotelian relations as being hybrid between two other types of relations, viz. the opposition and implication relations.

We will now define these two new types of relations. Recall that in Section 2, we showed that there are at least three distinct ways of defining the Aristotelian relations; in exactly the same way, there are also at least three ways of defining the opposition and implication relations. In this paper, however, we will only be dealing with these relations at the object-logical level (i.e. as holding between formulas of the language $\mathcal{L}_{\mathrm{S}}$ of some logical system S), and thus there is no need for the full generality that was provided by Definition 2.3. Hence, we can define the opposition and implication relations 
FiguRE 1. Code for visualizing (a) the opposition relations and (b) the implication relations

\begin{tabular}{|c|c|c|c|c|}
\hline contradiction & & & hi-imnlication & \\
\hline contrariety & $C$ & - & left-implication & $L I$ \\
\hline subcontrariety & $S C$ & 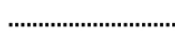 & right-implication & $R I$ \\
\hline non-contradiction & $N C D$ & 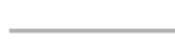 & non-implication & $N I$ \\
\hline
\end{tabular}

in a way that is analogous to Definition 2.2 (if desired, the analogues of Definitions 2.1 and 2.3 could easily be given too).

Definition 3.1. Let $\mathrm{S}$ be a logical system as in Definition 2.2. Two formulas $\varphi, \psi \in \mathcal{L}_{\mathrm{S}}$ are said to be

\begin{tabular}{|c|c|c|}
\hline contradictory & iff & $\mathrm{S} \models \neg(\varphi \wedge \psi)$ \\
\hline -contrary & iff & $\mathrm{S} \models \neg(\varphi \wedge \psi)$ \\
\hline subcontrary & & $\mathrm{S} \not \models \neg(\varphi \wedge \psi)$ \\
\hline -non-contradictory & iff & $\mathrm{S} \not \models \neg(\varphi \wedge \psi)$ \\
\hline
\end{tabular}

These relations are abbreviated as $C D_{\mathrm{S}}, C_{\mathrm{S}}, S C_{\mathrm{S}}$ and $N C D_{\mathrm{S}}$, respectively. The set $\mathcal{O} \mathcal{G}_{\mathrm{S}}$ consisting of these four relations is called the opposition geometry of S, i.e. $\mathcal{O G} \mathcal{G}_{\mathrm{S}}:=\left\{C D_{\mathrm{S}}, C_{\mathrm{S}}, S C_{\mathrm{S}}, N C D_{\mathrm{S}}\right\}$.

Definition 3.2. Let $S$ be a logical system as in Definition 2.2. Two formulas $\varphi, \psi \in \mathcal{L}_{\mathrm{S}}$ are said to be

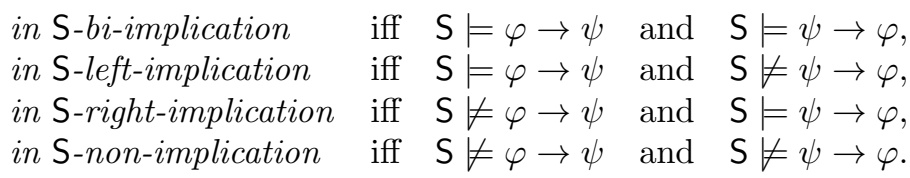

These relations are abbreviated as $B I_{\mathrm{S}}, L I_{\mathrm{S}}, R I_{\mathrm{S}}$ and $N I_{\mathrm{S}}$, respectively. The set $\mathcal{I} \mathcal{G}_{\mathrm{S}}$ consisting of these four relations is called the implication geometry of $\mathrm{S}$, i.e. $\mathcal{I G}_{\mathrm{S}}:=\left\{B I_{\mathrm{S}}, L I_{\mathrm{S}}, R I_{\mathrm{S}}, N I_{\mathrm{S}}\right\}$.

The opposition and implication relations will be visualized using the code shown in Figure 1. It should be noted that S-left-implication coincides with S-subalternation; consequently, the Aristotelian geometry $\mathcal{A G}_{\mathrm{S}}:=$ $\left\{C D_{\mathrm{S}}, C_{\mathrm{S}}, S C_{\mathrm{S}}, L I_{\mathrm{S}}\right\}$ is hybrid between the opposition and implication geometries, i.e. $\mathcal{A G}_{\mathrm{S}} \subseteq \mathcal{O G}_{\mathrm{S}} \cup \mathcal{I} \mathcal{G}_{\mathrm{S}}$. Furthermore, the Aristotelian geometry is hybrid in an informationally optimal fashion. To explain this claim, [79] introduces a well-motivated information measure $\mathfrak{m}$ that allows us to compare the information levels of any two opposition and/or implication relations. Next, note that any two formulas stand in exactly one opposition relation and in exactly one implication relation. Essentially, if the formulas happen to be S-contingent, it can be shown that one of these two relations is strictly 
Figure 2. The (a) opposition, (b) Aristotelian and (c) implication square for $\{\square p, \diamond p, \square \neg p, \diamond \neg p\}$
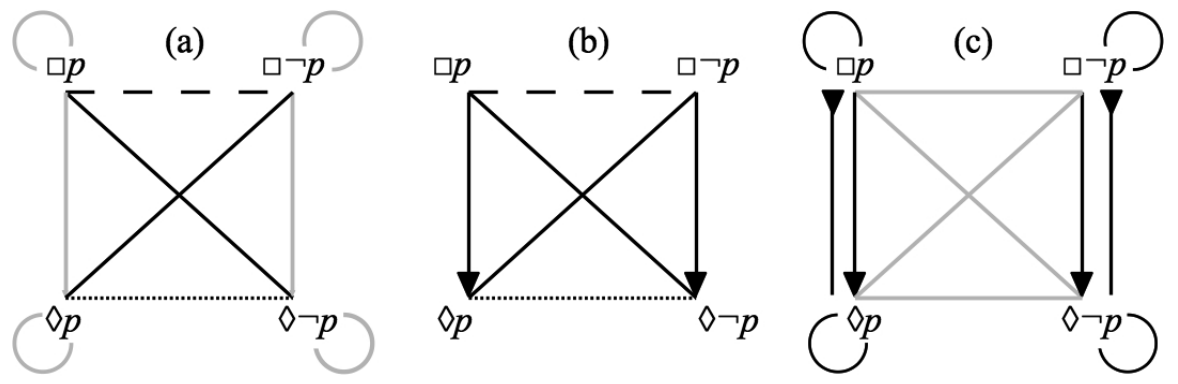

more informative than the other (according to $\mathfrak{m}$ ) iff it is Aristotelian [79, Section 5]..$^{2}$

To illustrate this, consider the modal logic S5 and the S5-contingent formulas $\square p, \diamond p, \square \neg p$ and $\diamond \neg p$. It is well-known that the Aristotelian relations holding between these formulas yield a classical Aristotelian square, which is shown in Figure 2(b). However, in exactly the same fashion, the opposition and implication relations holding between these formulas yield opposition and implication squares, which are shown in Figure 2(a) and (c), respectively. Now consider some pairs of formulas:

- $\square p$ and $\square \neg p$ : these stand in the opposition relation $C_{\mathrm{S} 5}$ and the implication relation $N I_{\mathrm{S} 5}$; according to $\mathfrak{m}, C_{\mathrm{S} 5}$ is strictly more informative than $N I_{\mathrm{S} 5}$, and $C_{\mathrm{S} 5}$ is an Aristotelian relation, while $N I_{\mathrm{S} 5}$ is not.

- $\diamond p$ and $\square \neg p$ : these stand in the opposition relation $C D_{\mathrm{S} 5}$ and the implication relation $N I_{\mathrm{S} 5}$; according to $\mathfrak{m}, C D_{\mathrm{S} 5}$ is strictly more informative than $N I_{\mathrm{S} 5}$, and $C D_{\mathrm{S} 5}$ is an Aristotelian relation, while $N I_{\mathrm{S} 5}$ is not.

- $\square p$ and $\diamond p$ : these stand in the opposition relation $N C D_{\mathrm{S} 5}$ and the implication relation $L I_{\mathrm{S} 5}$; according to $\mathfrak{m}, L I_{\mathrm{S} 5}$ is strictly more informative than $N C D_{\mathrm{S} 5}$, and $L I_{\mathrm{S} 5}$ is an Aristotelian relation, while $N C D_{\mathrm{S} 5}$ is not.

The Aristotelian square in Figure 2(b) is thus hybrid between the opposition and implication squares in Figure 2(a) and (c), respectively: every pair of formulas stands in an opposition relation and an implication relation, and the Aristotelian square visualizes exactly the more informative of these two relations.

The opposition and implication geometries were primarily introduced because of their relation to the Aristotelian geometry, but they also turn out to be very interesting in their own right. We now briefly mention some basic results from [79] that will be useful later in this paper.

Lemma 3.3. For all formulas $\varphi, \psi \in \mathcal{L}_{\mathrm{S}}$, the following hold:

\footnotetext{
${ }^{2}$ If neither of the two relations is strictly more informative than the other, then neither is Aristotelian, and thus the two formulas stand in no Aristotelian relation at all.
} 


$\begin{array}{lrlrlrll}\text { 1a) } & C D_{\mathrm{S}}(\varphi, \psi) & \text { iff } & C D_{\mathrm{S}}(\neg \varphi, \neg \psi), & 1 b) & B I_{\mathrm{S}}(\varphi, \psi) & \text { iff } & B I_{\mathrm{S}}(\neg \varphi, \neg \psi), \\ \text { 2a) } & C_{\mathrm{S}}(\varphi, \psi) & \text { iff } & S C_{\mathrm{S}}(\neg \varphi, \neg \psi), & 2 b) & L I_{\mathrm{S}}(\varphi, \psi) & \text { iff } & R I_{\mathrm{S}}(\neg \varphi, \neg \psi), \\ 3 a) & S C_{\mathrm{S}}(\varphi, \psi) & \text { iff } & C_{\mathrm{S}}(\neg \varphi, \neg \psi), & 3 b) & R I_{\mathrm{S}}(\varphi, \psi) & \text { iff } & L I_{\mathrm{S}}(\neg \varphi, \neg \psi), \\ \text { 4a) } & N C D_{\mathrm{S}}(\varphi, \psi) & \text { iff } & N C D_{\mathrm{S}}(\neg \varphi, \neg \psi), & 4 b) & N I_{\mathrm{S}}(\varphi, \psi) & \text { iff } & N I_{\mathrm{S}}(\neg \varphi, \neg \psi) .\end{array}$

Lemma 3.4. For all formulas $\varphi, \psi \in \mathcal{L}_{\mathrm{S}}$, the following hold:

$\begin{array}{lrlrlrlr}1 a) & C D_{\mathrm{S}}(\varphi, \psi) & \text { iff } & B I_{\mathrm{S}}(\neg \varphi, \psi), & 1 b) & C D_{\mathrm{S}}(\varphi, \psi) & \text { iff } & B I_{\mathrm{S}}(\varphi, \neg \psi), \\ 2 a) & C_{\mathrm{S}}(\varphi, \psi) & \text { iff } & R I_{\mathrm{S}}(\neg \varphi, \psi), & 2 b) & C_{\mathrm{S}}(\varphi, \psi) & \text { iff } & L I_{\mathrm{S}}(\varphi, \neg \psi), \\ 3 a) & S C_{\mathrm{S}}(\varphi, \psi) & \text { iff } & L I_{\mathrm{S}}(\neg \varphi, \psi), & 3 b) & S C_{\mathrm{S}}(\varphi, \psi) & \text { iff } & R I_{\mathrm{S}}(\varphi, \neg \psi), \\ 4 a) & N C D_{\mathrm{S}}(\varphi, \psi) & \text { iff } & N I_{\mathrm{S}}(\neg \varphi, \psi), & 4 b) & N C D_{\mathrm{S}}(\varphi, \psi) & \text { iff } & N I_{\mathrm{S}}(\varphi, \neg \psi), \\ 5 a) & B I_{\mathrm{S}}(\varphi, \psi) & \text { iff } & C D_{\mathrm{S}}(\neg \varphi, \psi), & 5 b) & B I_{\mathrm{S}}(\varphi, \psi) & \text { iff } & C D_{\mathrm{S}}(\varphi, \neg \psi), \\ 6 a) & L I_{\mathrm{S}}(\varphi, \psi) & \text { iff } & S C_{\mathrm{S}}(\neg \varphi, \psi), & 6 b) & L I_{\mathrm{S}}(\varphi, \psi) & \text { iff } & C_{\mathrm{S}}(\varphi, \neg \psi), \\ 7 a) & R I_{\mathrm{S}}(\varphi, \psi) & \text { iff } & C_{\mathrm{S}}(\neg \varphi, \psi), & 7 b) & R I_{\mathrm{S}}(\varphi, \psi) & \text { iff } & S C_{\mathrm{S}}(\varphi, \neg \psi), \\ 8 a) & N I_{\mathrm{S}}(\varphi, \psi) & \text { iff } & N C D_{\mathrm{S}}(\neg \varphi, \psi), & 8 b) & N I_{\mathrm{S}}(\varphi, \psi) & \text { iff } & N C D D_{\mathrm{S}}(\varphi, \neg \psi) .\end{array}$

Lemma 3.5. For all formulas $\varphi, \psi \in \mathcal{L}_{\mathrm{S}}$, the following hold:

1. there is exactly one $R \in \mathcal{O G}_{\mathrm{S}}$ such that $R(\varphi, \psi)$,

2. there is exactly one $S \in \mathcal{I G}_{\mathrm{S}}$ such that $S(\varphi, \psi)$.

Lemma 3.5 essentially states that $\mathcal{O} \mathcal{G}_{\mathrm{S}}$ and $\mathcal{I} \mathcal{G}_{\mathrm{S}}$ are partitions of $\mathbb{B}(\mathrm{S}) \times$ $\mathbb{B}(S)$ (recall that $\mathbb{B}(S)$ denotes the Lindenbaum-Tarski algebra of $S$ ). In the contemporary study of Aristotelian diagrams, partitions play an important role, because of their intimate connection to bitstring semantics [25, 30, 82]. In this paper, this connection will not be explored in any detail; we will suffice by briefly describing the analogy between the partition-based bitstring semantics for classical propositional logic ( $\mathrm{CPL}$ ) and the partition-based bitstring semantics for the opposition and implication relations.

In CPL, the formulas $p$ and $q$ induce the partition $\{p \wedge q, p \wedge \neg q, \neg p \wedge$ $q, \neg p \wedge \neg q$ \} of the class of all CPL-models (cf. the 4 rows in a truth table for a binary propositional connective); therefore, all Boolean combinations of formulas in this partition can be represented by bitstrings of length 4 . Entirely analogously, the sets $\{([\varphi],[\psi]) \mid \mathrm{S} \models \neg(\varphi \wedge \psi)\}$ and $\{([\varphi],[\psi]) \mid \mathrm{S} \models$ $\varphi \vee \psi\}$ induce the partition $\left\{C D_{\mathrm{S}}, C_{\mathrm{S}}, S C_{\mathrm{S}}, N C D_{\mathrm{S}}\right\}=\mathcal{O} \mathcal{G}_{\mathrm{S}}$ of $\mathbb{B}(\mathrm{S}) \times \mathbb{B}(\mathrm{S})$; therefore, in this partition as well, all Boolean combinations of relations can be represented by bitstrings of length 4 . Similar remarks apply to $\mathcal{I G}_{\mathrm{S}}$.

\subsection{The Duality Relations}

The final set of logical relations that we will study consists of the well-known duality relations. Like the Aristotelian relations (but unlike the opposition and implication relations), the duality relations will be used in this paper at both the object- and the metalogical level, i.e. as holding between formulas/operators of the language $\mathcal{L}_{\mathrm{S}}$ of some logical system $\mathrm{S}$ as well as between metalogical statements/operators. They will therefore be defined in a fully general way (analogous to Definition 2.3 of the Aristotelian relations).

Definition 3.6. Consider Boolean algebras $\mathbb{A}=\left\langle A, \wedge_{\mathbb{A}}, \vee_{\mathbb{A}}, \neg_{\mathbb{A}}, \top_{\mathbb{A}}, \perp_{\mathbb{A}}\right\rangle$ and $\mathbb{B}=\left\langle B, \wedge_{\mathbb{B}}, \vee_{\mathbb{B}}, \neg_{\mathbb{B}}, \top_{\mathbb{B}}, \perp_{\mathbb{B}}\right\rangle$. The duality relations between $n$-ary operators $O_{1}, O_{2}: \mathbb{A}^{n} \rightarrow \mathbb{B}$ are defined as follows: 
Figure 3. (a) Code for visualizing the duality relations; (b) duality square for $\{p \wedge q, \neg p \wedge \neg q, p \vee q, \neg p \vee \neg q\}$

(a)

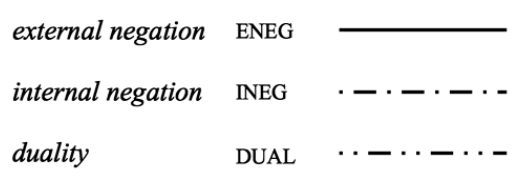

(b)

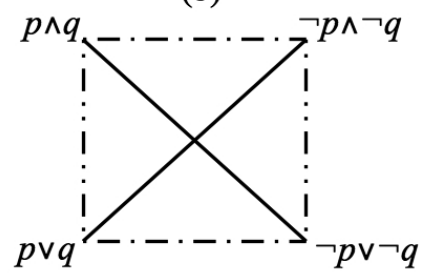

- $O_{1}$ and $O_{2}$ are identical iff $\forall a_{1}, \ldots, a_{n} \in A: O_{1}\left(a_{1}, \ldots, a_{n}\right)=O_{2}\left(a_{1}, \ldots, a_{n}\right)$,

- $O_{1}$ and $O_{2}$ are each other's external negation iff $\forall a_{1}, \ldots, a_{n} \in A: O_{1}\left(a_{1}, \ldots, a_{n}\right)=\neg_{\mathbb{B}} O_{2}\left(a_{1}, \ldots, a_{n}\right)$,

- $O_{1}$ and $O_{2}$ are each other's internal negation iff $\forall a_{1}, \ldots, a_{n} \in A: O_{1}\left(a_{1}, \ldots, a_{n}\right)=O_{2}\left(\neg_{\mathbb{A}} a_{1}, \ldots, \neg_{\mathbb{A}} a_{n}\right)$,

- $O_{1}$ and $O_{2}$ are each other's dual iff $\forall a_{1}, \ldots, a_{n} \in A: O_{1}\left(a_{1}, \ldots, a_{n}\right)=\neg_{\mathbb{B}} O_{2}\left(\neg_{\mathbb{A}} a_{1}, \ldots, \neg_{\mathbb{A}} a_{n}\right)$.

These relations are abbreviated as ID, ENEG, INEG and DUAL, respectively. The set $\mathcal{D G}$ consisting of these four relations is called the duality geometry , i.e. $\mathcal{D G}:=\{$ ID, ENEG, INEG, DUAL $\}$.

Although the duality relations are strictly speaking defined between operators $O_{1}, O_{2}: \mathbb{A}^{n} \rightarrow \mathbb{B}$, it will be convenient to also be able to apply them to the elements of $\mathbb{B}$ directly: we will say that $O_{1}\left(a_{1}, \ldots, a_{n}\right)$ and $O_{2}\left(a_{1}, \ldots, a_{n}\right)$ stand in some duality relation $R$ iff the operators $O_{1}$ and $\mathrm{O}_{2}$ stand in $R$. Consider, for example, conjunction and disjunction in classical propositional logic $(\mathrm{CPL})$ : these can be viewed as binary operators $\wedge, \vee: \mathbb{B}(\mathrm{CPL}) \times \mathbb{B}(\mathrm{CPL}) \rightarrow \mathbb{B}(\mathrm{CPL})$. It trivially holds for all $\varphi, \psi \in \mathcal{L}_{\mathrm{CPL}}$ that $\varphi \wedge \psi \equiv \mathrm{CPL} \neg(\neg \varphi \vee \neg \psi)$, so by Definition 3.6 we have $\operatorname{DUAL}(\wedge, \vee)$. Moving from operators to concrete formulas, we can also say that $\operatorname{DUAL}(p \wedge q, p \vee q)$ and $\operatorname{DUAL}(\neg p \wedge \neg q, \neg p \vee \neg q)$. In exactly the same way, it can be shown that $\operatorname{INEG}(p \wedge q, \neg p \wedge \neg q), \operatorname{INEG}(p \vee q, \neg p \vee \neg q), \operatorname{ENEG}(p \wedge q, \neg p \vee \neg q)$ and $\operatorname{ENEG}(p \vee q, \neg p \wedge \neg q)$. Using the visual code shown in Figure 3(a), we thus obtain the duality square shown in Figure 3(b).

When viewed as relations between elements of $\mathbb{B}(\mathrm{S})$, the duality relations ID and ENEG correspond exactly to the implication relation $B I_{\mathrm{S}}$ and the opposition relation $C D_{\mathrm{S}}$, respectively. Furthermore, looking at the duality square in Figure $3(\mathrm{~b})$, it looks like DUAL corresponds to $L I_{\mathrm{S}}$, while INEG corresponds to both $C_{\mathrm{S}}$ and $S C_{\mathrm{S}}$. Observations such as these might explain why some authors [20,58] have come close to straightforwardly identifying Aristotelian and duality squares, for example by using Aristotelian terminology to describe a duality square (or vice versa), or by viewing one as a generalization of the other. 
However, it has also been argued extensively that although $\mathcal{A G}$ and $\mathcal{D G}$ sometimes yield similar-looking diagrams, they are two conceptually independent sets of logical relations, and should thus not be confused with each other $[29,54,77,84]$. For example, it is easy to show that all duality relations are symmetric, i.e. for all $R \in \mathcal{D} \mathcal{G}$ and for all operators $O_{1}, O_{2}$, it holds that $R\left(O_{1}, O_{2}\right)$ iff $R\left(O_{2}, O_{1}\right)$; this clearly shows that DUAL cannot be identified with $L I_{\mathrm{S}}$, since the latter is asymmetric. Another important difference is that unlike the Aristotelian relations, the duality relations are functional, i.e. for all $R \in \mathcal{D G}$ and for all operators $O_{1}$, there exists a unique operator $O_{2}$ such that $R\left(O_{1}, O_{2}\right) .{ }^{3}$ The duality relation $R$ can thus be seen as a function, and we will often write $O_{2}=R\left(O_{1}\right)$. For example, since $\operatorname{DUAL}(\wedge, \vee)$, we can also say that $\operatorname{DUAL}(\wedge)=\vee$. Since the duality relations are symmetric, the corresponding functions are idempotent, i.e. $R\left(R\left(O_{1}\right)\right)=O_{1}$ for all $R \in \mathcal{D G}$ and operators $O_{1} ;{ }^{4}$ in other words: $R \circ R=$ ID. More generally, it is well-known that when its elements are viewed as functions, $\mathcal{D G}$ forms a Klein four group $[36,53,83,65,66]$, which has the following Cayley table:

\begin{tabular}{c|cccc}
$\circ$ & ID & ENEG & INEG & DUAL \\
\hline ID & ID & ENEG & INEG & DUAL \\
ENEG & ENEG & ID & DUAL & INEG \\
INEG & INEG & DUAL & ID & ENEG \\
DUAL & DUAL & INEG & ENEG & ID
\end{tabular}

This Cayley table and the duality square in Figure 4(a) might suggest that $\operatorname{ID}(O), \operatorname{ENEG}(O)$, INEG $(O)$ and $\operatorname{DUAL}(O)$ are pairwise distinct for all operators $O$. However, there are also exist operators $O$ that are their own dual, i.e. $\operatorname{DuAL}(O)=O=\operatorname{ID}(O)$; it then follows that also $\operatorname{EnEG}(O)=\operatorname{InEG}(O)$, and thus the duality square in Figure 4(a) degenerates into the horizontal duality diagram in Figure 4(b). Viewing the elements of $\mathcal{D} \mathcal{G}$ as relations, we thus find that ID $\cap$ DUAL $\neq \emptyset \neq$ ENEG $\cap$ INEG. A typical example of an operator that is its own dual is negation $\neg: \mathbb{B}(\mathrm{S}) \rightarrow \mathbb{B}(\mathrm{S})$, since $\neg \varphi \equiv \mathrm{S} \neg \neg \neg \varphi$ for all $\varphi \in \mathcal{L}_{S}$. Completely analogously, there also exist operators $O$ that are their own internal negation, i.e. $\operatorname{INEG}(O)=O=\operatorname{ID}(O)$; it then follows that also $\operatorname{EnEG}(O)=\operatorname{DUAL}(O)$, and thus the duality square in Figure 4(a) degenerates into the vertical duality diagram in Figure 4(c). Again viewing the elements of $\mathcal{D G}$ as relations, we find that ID $\cap$ INEG $\neq \emptyset \neq$ ENEG $\cap$ DUAL. A typical example of an operator that is its own internal negation is the biconditional $\leftrightarrow: \mathbb{B}(\mathrm{CPL}) \times \mathbb{B}(\mathrm{CPL}) \rightarrow \mathbb{B}(\mathrm{CPL})$, since $\varphi \leftrightarrow \psi \equiv \mathrm{CPL} \neg \varphi \leftrightarrow \neg \psi$ for all $\varphi, \psi \in \mathcal{L}_{\mathrm{CPL}} .^{5}$

\footnotetext{
${ }^{3}$ For example, the formula $p \wedge q$ is contrary to many formulas (e.g. to $\neg p$, to $\neg q$, to $\neg p \wedge \neg q$, etc.), but it has only one internal negation (viz. $\neg p \wedge \neg q$ ).

${ }^{4}$ Proof: $R\left(O_{1}\right)=O_{2} \Leftrightarrow R\left(O_{1}, O_{2}\right) \Leftrightarrow R\left(O_{2}, O_{1}\right) \Leftrightarrow R\left(O_{2}\right)=O_{1}$, and thus $R\left(R\left(O_{1}\right)\right)=$ $R\left(O_{2}\right)=O_{1}$.

${ }^{5}$ After having dealt with operators that coincide with their own dual or their own internal negation, one might wonder whether there are also operators that coincide with their own external negation. However, it is easy to show that if an operator $O: \mathbb{A}^{n} \rightarrow \mathbb{B}$ satisfies $\operatorname{ENEG}(O)=O$, then $\mathbb{B}$ has to be the trivial Boolean algebra in which $\perp_{\mathbb{B}}=\mathrm{\top}_{\mathbb{B}}$.
} 
Figure 4. (a) Non-degenerated duality square, (b) degenerated duality diagram for operators that that are their own dual, (c) degenerated duality diagram for operators that are their own internal negation

(a)

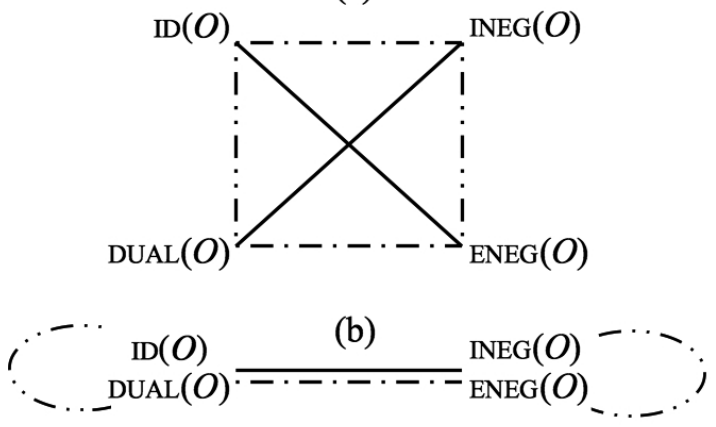

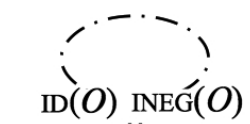

(c)

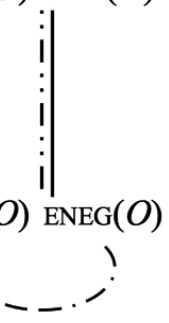

The basic perspective on duality that has been described so far can be extended and generalized in various ways. For example, duality patterns for composed operators are studied in [21]. Another generalization, which will turn out to be very useful in Section 8, is the so-called generalized Post duality $[41,51]$. Recall that according to Definition 3.6, the INEG-relation involves negating all the operator's arguments. Although the most canonical examples of duality indeed obey this requirement - e.g. the internal negation of $p \wedge q$ is $\neg p \wedge \neg q$; see Figure $3(\mathrm{~b})$ - , there are also important examples in which internal negation is applied to only one of the operator's arguments, such as generalized quantifiers (on the relational perspective), subject negation, and the public announcement operator [22, 29, 37, 45, 47]. Generalized Post duality accommodates these examples, by 'splitting' the INEG-relation into $n$ independent relations $\mathrm{INEG}_{1}, \ldots, \mathrm{INEG}_{n}$; as a consequence, the DUAL-relation is also split into $n$ independent relations $\mathrm{DUAL}_{1}, \ldots, \mathrm{DUAL}_{n}$.

Definition 3.7. Consider Boolean algebras $\mathbb{A}=\left\langle A, \wedge_{\mathbb{A}}, \vee_{\mathbb{A}}, \neg_{\mathbb{A}}, \top_{\mathbb{A}}, \perp_{\mathbb{A}}\right\rangle$ and $\mathbb{B}=\left\langle B, \wedge_{\mathbb{B}}, \vee_{\mathbb{B}}, \neg_{\mathbb{B}}, \top_{\mathbb{B}}, \perp_{\mathbb{B}}\right\rangle$. The generalized Post duality relations between $n$-ary operators $O_{1}, O_{2}: \mathbb{A}^{n} \rightarrow \mathbb{B}$ are defined as follows:

- ID and ENEG are defined as before (Definition 3.6),

- for $1 \leq i \leq n$, we define $\operatorname{INEG}_{i}\left(O_{1}, O_{2}\right): \Leftrightarrow$ $\forall a_{1}, \ldots, a_{n} \in A: O_{1}\left(a_{1}, \ldots, a_{n}\right)=O_{2}\left(a_{1}, \ldots, a_{i-1}, \neg_{\mathbb{A}} a_{i}, a_{i+1}, \ldots, a_{n}\right)$,

- for $1 \leq i \leq n$, we define $\operatorname{DUAL}_{i}\left(O_{1}, O_{2}\right): \Leftrightarrow$ $\forall a_{1}, \ldots, a_{n} \in A: O_{1}\left(a_{1}, \ldots, a_{n}\right)=\neg_{\mathbb{B}} O_{2}\left(a_{1}, \ldots, a_{i-1}, \neg_{\mathbb{A}} a_{i}, a_{i+1}, \ldots, a_{n}\right)$.

From the perspective of generalized Post duality, an $n$-ary operator $O$ thus possesses $n+1$ independent negation positions, viz. 1 external negation and $n$ internal negations. Consequently, if $n>1$, the generalized Post duality behavior of such an $n$-ary operator cannot be visualized by means of a simple 
Figure 5. (a) Generalized Post duality cube, and (b-c) two generalized Post duality squares

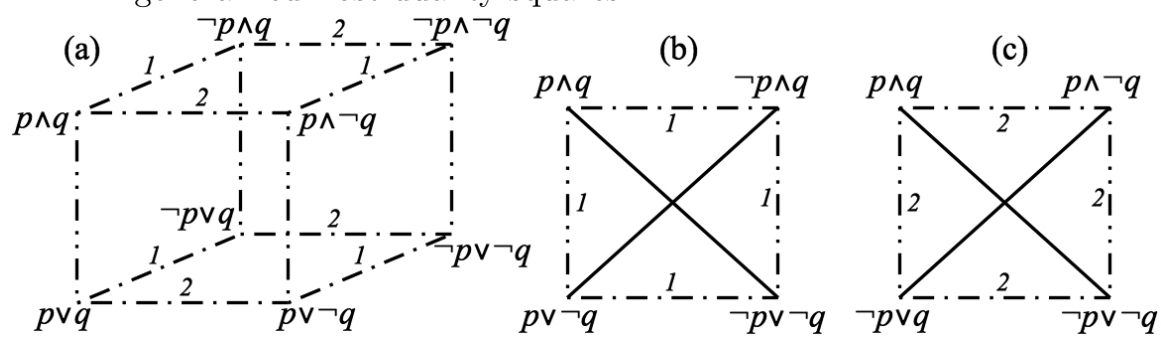

square diagram, but rather requires an $(n+1)$-dimensional hypercube. For the binary operator of conjunction, for instance, we have $\operatorname{INEG}_{1}(p \wedge q, \neg p \wedge q)$, $\mathrm{DUAL}_{1}(p \wedge q, p \vee \neg q), \operatorname{INEG}_{2}(p \wedge q, p \wedge \neg q), \operatorname{DUAL}_{2}(p \wedge q, \neg p \vee q)$, etc.; all these facts can be visualized by means of a three-dimensional generalized Post du-

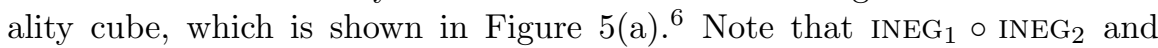
ENEG $\circ \mathrm{INEG}_{1} \circ \mathrm{INEG}_{2}$ are the classical INEG- and DUAL-relations, respectively. Furthermore, it can be shown group-theoretically that the generalized Post duality cube in Figure 5(a) contains exactly 14 duality squares as subdiagrams [21], two of which are shown in Figure 5(b-c).

Finally, it should be noted that there are binary operators $O$ such that $\operatorname{INEG}_{1}(O)=\operatorname{INEG}_{2}(O)$ (in this case, it also holds that $O$ is its own 'classical' internal negation, since $\operatorname{INEG}(O)=\left(\mathrm{INEG}_{1} \circ \mathrm{INEG}_{2}\right)(O)=\left(\mathrm{INEG}_{1} \circ\right.$ $\left.\left.\mathrm{INEG}_{1}\right)(O)=\mathrm{ID}(O)=O\right)$. For example, for the operator $O: \mathbb{B}(\mathrm{S} 5) \times \mathbb{B}(\mathrm{S} 5) \rightarrow$ $\mathbb{B}(\mathrm{S} 5):(\varphi, \psi) \mapsto \square(\varphi \leftrightarrow \psi)$ it holds that $\operatorname{INEG}_{1}(O)=\operatorname{INEG}_{2}(O)$, since $\square(\neg \varphi \leftrightarrow \psi) \equiv_{\mathrm{S} 5} \square(\varphi \leftrightarrow \neg \psi) .{ }^{7}$ In such cases, the generalized Post duality cube degenerates into a generalized Post duality square, which is shown in Figure 6(a). If we ignore the classical INEG- and DUAL-relations, this square can be decomposed into a square for $\mathrm{INEG}_{1} / \mathrm{DUAL}_{1}$ and one for $\mathrm{INEG}_{2} / \mathrm{DUAL}_{2}$, which are shown in Figure 6(b) and (c), respectively.

\footnotetext{
${ }^{6}$ Note that in case of generalized duality diagrams, we attach subscripts to the diagram's INEG- and DUAL-edges to indicate which $\mathrm{INEG}_{i}$ or $\mathrm{DUAL}_{i}$-relation they represent. If an INEGor DUAL-edge does not have any subscript, it still represents the classical INEG- or DUALrelation, respectively. Finally, note that in the cube in Figure 5(a) only INEG 1 , INEG2, and DUAL have been visualized, for reasons of visual simplicity (ENEG corresponds to the long diagonals of the cube, INEG corresponds to the diagonals of the top and bottom faces of the cube, DUAL 1 corresponds to the diagonals of the front and back faces of the cube, and DUAL $_{2}$ corresponds to the diagonals of the left and right faces of the cube).

${ }^{7}$ One might wonder why we do not stick to the simpler example of the biconditional $(\leftrightarrow)$, since it also holds that $\mathrm{INEG}_{1}(\leftrightarrow)=\mathrm{INEG}_{2}(\leftrightarrow)$. However, for the biconditonal we even have $\operatorname{INEG}_{1}(\leftrightarrow)=\operatorname{INEG}_{2}(\leftrightarrow)=\operatorname{ENEG}(\leftrightarrow)$, and thus its generalized Post duality cube does not simply degenerate into a square, but even further, into a binary duality diagram resembling the one shown in Figure 4(c).
} 
Figure 6. (a) Degenerated generalized Post duality cube for binary operators whose INEG $_{1}$ and $\mathrm{INEG}_{2}$ coincide, and its decomposition into two generalized Post duality squares for (b) $\mathrm{INEG}_{1} / \mathrm{DUAL}_{1}$ and (c) $\mathrm{INEG}_{2} / \mathrm{DUAL}_{2}$

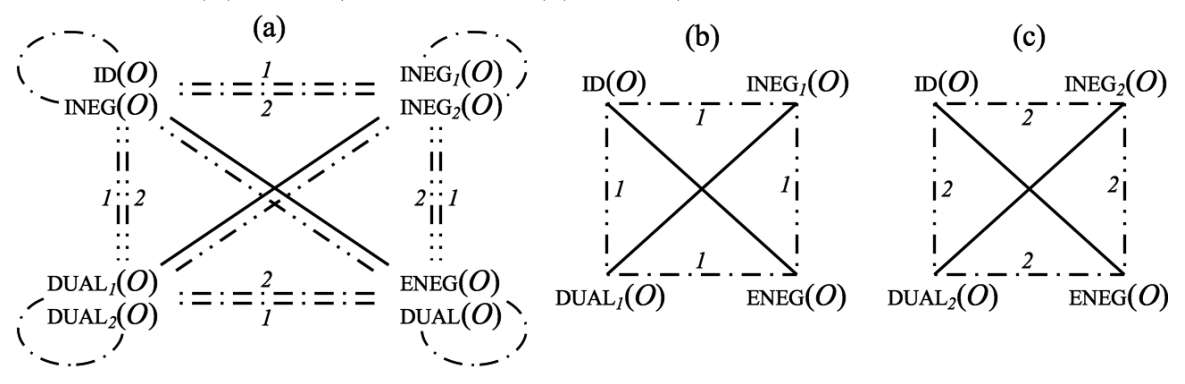

\section{Aristotelian Diagrams for the Opposition Relations}

We have now arrived at the core sections of the paper, which will be devoted to constructing and studying various metalogical decorations of various kinds of logical diagrams. In this section, we will study Aristotelian diagrams for the opposition geometry $(\mathcal{O G}) .{ }^{8}$ Subsection 4.1 introduces an Aristotelian rhombic dodecahedron for $\mathcal{O G}$. Subsections 4.2-4.5 study a number of interesting subdiagrams of this rhombic dodecahedron, and discuss their connections with earlier work on metalogical decorations of Aristotelian diagrams.

\subsection{An Aristotelian Rhombic Dodecahedron for the Opposition Relations}

It is well-known that if we take the Boolean closure of the CPL-fragment $\{p \wedge q, p \wedge \neg q, \neg p \wedge q, \neg p \wedge \neg q\}$, we get a Boolean algebra of 16 formulas, viz. the binary connectives applied to the propositional atoms $p$ and $q$. This Boolean algebra can be visualized by means of an ordinary (two-dimensional) Hasse diagram. It was shown in [85] that this Hasse diagram can also be visualized as a three-dimensional polyhedron, viz. a rhombic dodecahedron $(\mathrm{RDH})$. In [55, 78], however, it is shown that (a variant of) the RDH can also be used to visualize the Aristotelian relations holding in this Boolean algebra. Furthermore, the Hasse RDH and the Aristotelian RDH turn out to be intimately related to each other (this was already suggested in [74]; the mathematical details are worked out in [28]).

Given the close analogy between the CPL-fragment $\{p \wedge q, p \wedge \neg q, \neg p \wedge$ $q, \neg p \wedge \neg q\}$ and the opposition geometry $\mathcal{O G}=\{C D, C, S C, N C D\}$ that was described at the end of Subsection 3.1 (in particular, see Lemma 3.5), it should not be surprising that very similar results can be obtained for $\mathcal{O G}$. We begin by considering the Boolean closure of $\mathcal{O G}$, i.e. $\wp^{\cup}(\mathcal{O G}):=\{\bigcup \mathcal{X} \mid \mathcal{X} \subseteq$ $\mathcal{O G}$. This is a Boolean algebra with 16 elements; its bottom element is the empty relation over $\mathbb{B}(\mathrm{S})$, and its top element is $C D \cup C \cup S C \cup N C D=\mathbb{B}(\mathrm{S}) \times$

\footnotetext{
${ }^{8}$ Throughout this section (and the next ones), we will usually omit reference to the logical system $\mathrm{S}$, and thus simply write $\mathcal{O G}$ instead of $\mathcal{O} \mathcal{G}_{\mathrm{S}}, C D$ instead of $C D_{\mathrm{S}}$, etc.
} 
Figure 7. Two-dimensional Hasse diagram for $\wp^{\cup}(\mathcal{O G})$

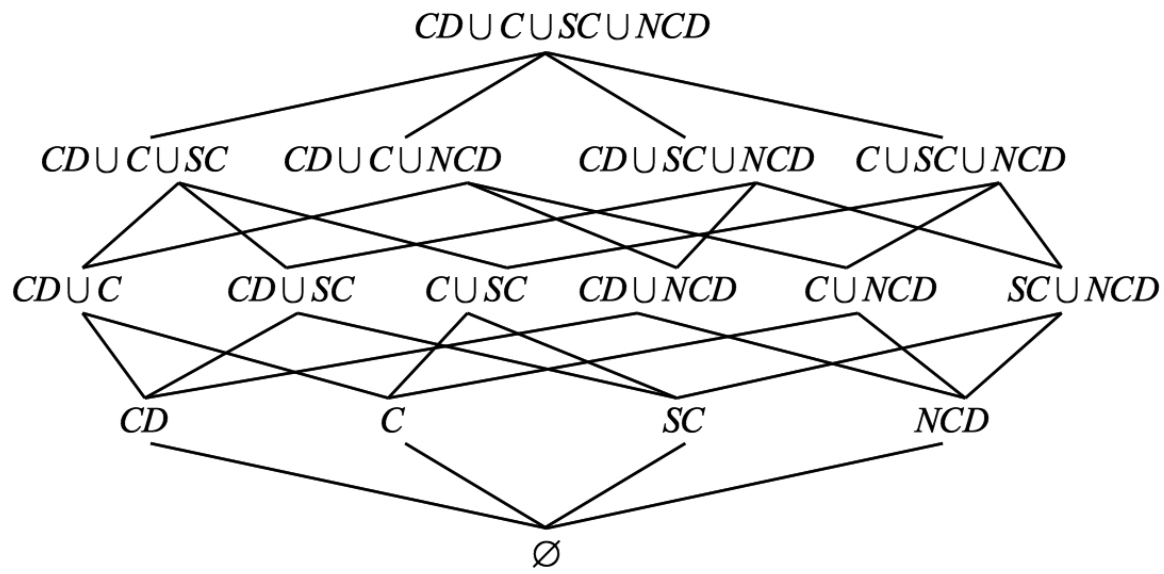

Figure 8. (a) Hasse and (b) Aristotelian rhombic dodecahedron for $\wp^{\cup}(\mathcal{O G})$

(a)

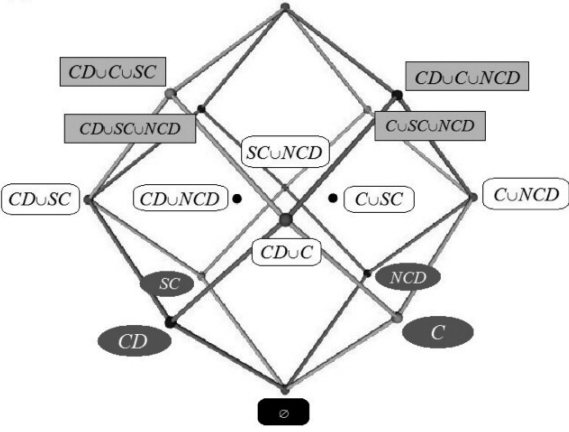

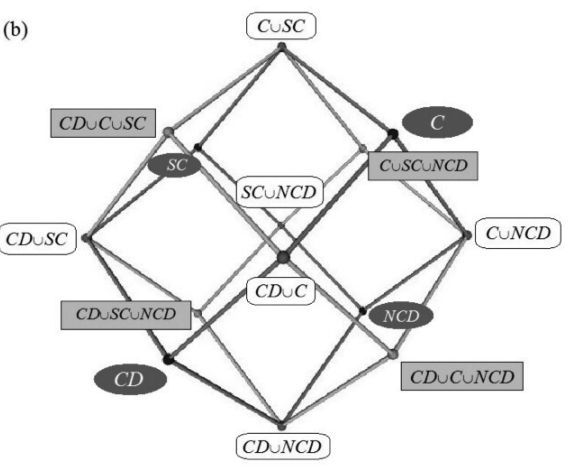

$\mathbb{B}(\mathrm{S})$, i.e. the universal relation over $\mathbb{B}(\mathrm{S})$. Figure 7 shows a (two-dimensional) Hasse diagram for $\wp(\mathcal{O G})$. Following Zellweger's [85] suggestion, this Hasse diagram can also be visualized as a rhombic dodecahedron; this Hasse RDH for $\wp^{\cup}(\mathcal{O G})$ is shown in Figure 8(a). The corresponding Aristotelian RDH is shown in Figure $8(\mathrm{~b}) .^{9}$

The elements in the Hasse RDH and the Aristotelian RDH are not formulas of some logical system $S$ - i.e. elements of $\mathbb{B}(S)$ - , but rather binary

\footnotetext{
${ }^{9}$ Note that the Aristotelian RDH in Figure 8(b) contains only 14 relations: like almost all Aristotelian diagrams in the literature, it does not contain $\wp^{\cup}(\mathcal{O G})$ 's bottom element $\emptyset$ and top element $\mathbb{B}(\mathrm{S}) \times \mathbb{B}(\mathrm{S})$. (It has been suggested that these elements are actually not absent from Aristotelian diagrams, but should rather be thought of as coinciding in the diagrams' centers of symmetry [69, 74]; recently, it has been shown that this suggestion is essentially correct, and can be derived from a general mathematical account of the relationship between Hasse diagrams and Aristotelian diagrams [28].)
} 
relations defined over that system-i.e. elements of $\wp(\mathbb{B}(S) \times \mathbb{B}(S))$ - ; both of these RDHs are thus metalogical diagrams. In particular, the Aristotelian $\mathrm{RDH}$ for $\mathcal{O} \mathcal{G}$ in Figure 8(b) is by far the largest and most complex Aristotelian diagram with a metalogical decoration that has been studied thus far.

The internal structure of the Aristotelian $\mathrm{RDH}$ for $\mathcal{O} \mathcal{G}$ can best be described in terms of its subdiagrams. For example, it is well-known that every Aristotelian RDH-regardless of its decoration-contains exactly six strong Jacoby-Sesmat-Blanché hexagons [55, 69, 74, 80]. ${ }^{10}$ Figure 9 shows what these hexagons look like in the concrete case of the Aristotelian RDH for $\mathcal{O G}$. In ongoing research $[32,81]$, we are working on an exhaustive typology of all subdiagrams that can be found inside an Aristotelian RDH (again, regardless of its decoration). All these results can then straightforwardly be applied to the specific case of the Aristotelian $\mathrm{RDH}$ for $\mathcal{O} \mathcal{G}$. In the next few subsections, however, we will not strive for such exhaustiveness, but rather focus on some specific subdiagrams of this Aristotelian RDH that turn out to be particularly interesting.

\subsection{Béziau's Hexagon for Tautology and Related Metalogical Notions}

One of the subdiagrams of the Aristotelian $\mathrm{RDH}$ for $\mathcal{O} \mathcal{G}$ that turns out to be particularly interesting is the JSB hexagon shown in Figure 10(a). This diagram does not occur in the list of six given in Figure 9, because it is a weak JSB hexagon; after all, the join of its contrary elements is $C \cup S C \cup N C D$, which is not the top element of $\wp^{\cup}(\mathcal{O G})$. Furthermore, this hexagon can also be reformulated using metalogical statements about formulas $\varphi, \psi \in \mathcal{L}_{\mathrm{S}}$, as shown in Figure 10(b).

Let's now see what happens if we fill in the same formula $\varphi$ twice in these metalogical statements, as is shown in Figure 11(a). In terms of relations, this means that we are intersecting each relation $R \in \wp^{\cup}(\mathcal{O G})$ with the identity relation $\Delta:=\left\{([\varphi],[\varphi]) \mid \varphi \in \mathcal{L}_{\mathrm{S}}\right\}$ on $\mathbb{B}(\mathrm{S})$, and are thus no longer working in the Boolean algebra $\wp^{\cup}(\mathcal{O G})$, but rather in the Boolean algebra $\wp^{\cup}(\{R \cap \Delta \mid R \in \mathcal{O G}\})=\{(\bigcup \mathcal{X}) \cap \Delta \mid \mathcal{X} \subseteq \mathcal{O G}\}$. Assuming that the underlying logical system $\mathrm{S}$ is consistent, ${ }^{11}$ we get:

$$
\begin{array}{rllll}
C D(\varphi, \varphi) & \text { iff } S \models \neg(\varphi \wedge \varphi) \text { and } S \models \varphi \vee \varphi & \text { iff } & \text { impossible, } \\
C(\varphi, \varphi) & \text { iff } S \models \neg(\varphi \wedge \varphi) \text { and } S \not \models \vee \varphi & \text { iff } & \varphi \text { is a contradiction, } \\
S C(\varphi, \varphi) & \text { iff } S \not \models \neg(\varphi \wedge \varphi) \text { and } S \models \varphi \vee \varphi & \text { iff } & \varphi \text { is a tautology, } \\
N C D(\varphi, \varphi) & \text { iff } S \not \models \neg(\varphi \wedge \varphi) \text { and } S \not \models \varphi \vee \varphi & \text { iff } & \varphi \text { is a contingency. }
\end{array}
$$

\footnotetext{
${ }^{10}$ The Jacoby-Sesmat-Blanché (JSB) hexagons are so-called because they were first studied in the 1950s by Jacoby [43], Sesmat [70] and Blanché [10, 11, 12, 13] (back then not with metalogical decorations, of course). The distinction between strong and weak JSB hexagons was first introduced by Pellissier [64]. Using the terminology of Definition 2.3, a JSB hexagon is said to be strong iff the join of its three contrary elements is the top element of the Boolean algebra in which it is defined; it is said to be weak otherwise. For example, the JSB hexagon in Figure 9 (a) is strong, because its contrary elements are $C D$, $C$ and $S C \cup N C D$, and their join $C D \cup C \cup(S C \cup N C D)$ is indeed the top element of $\wp \cup(\mathcal{O G})$.

${ }^{11}$ We will return to this assumption at the end of this subsection.
} 
Figure 9. The six strong Jacoby-Sesmat-Blanché hexagons that are subdiagrams of the Aristotelian $\mathrm{RDH}$ for $\mathcal{O G}$
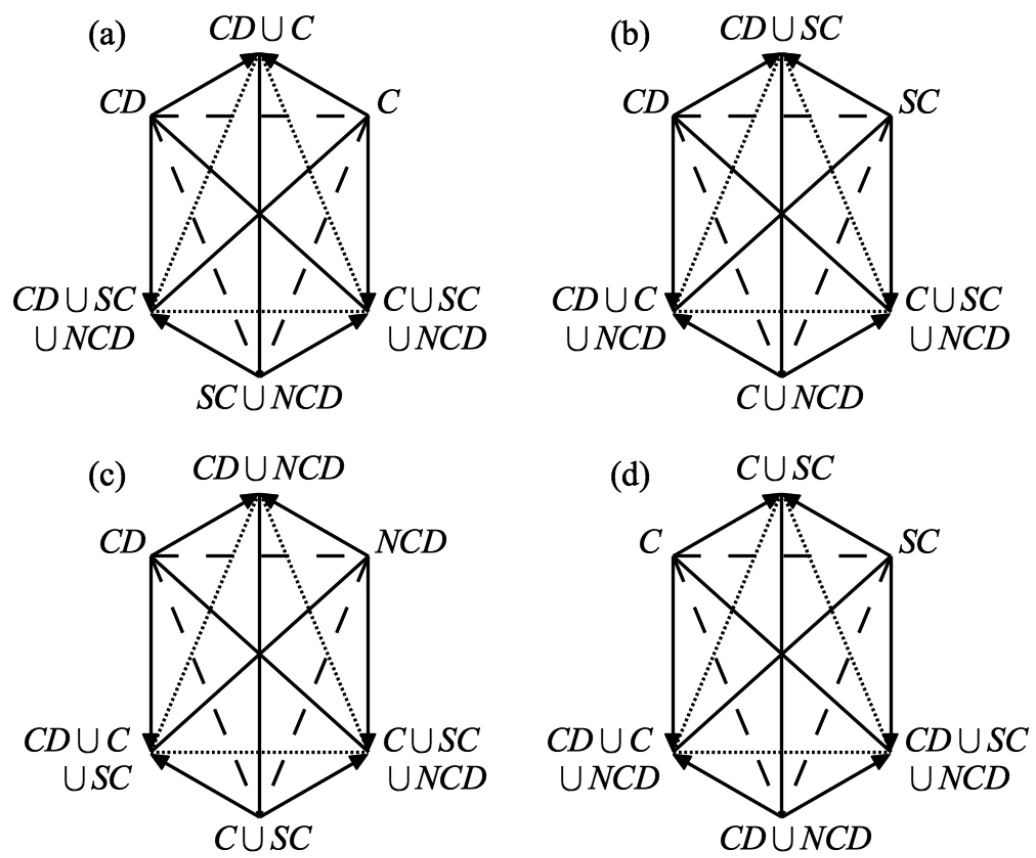

(e) $\quad C \cup N C D$
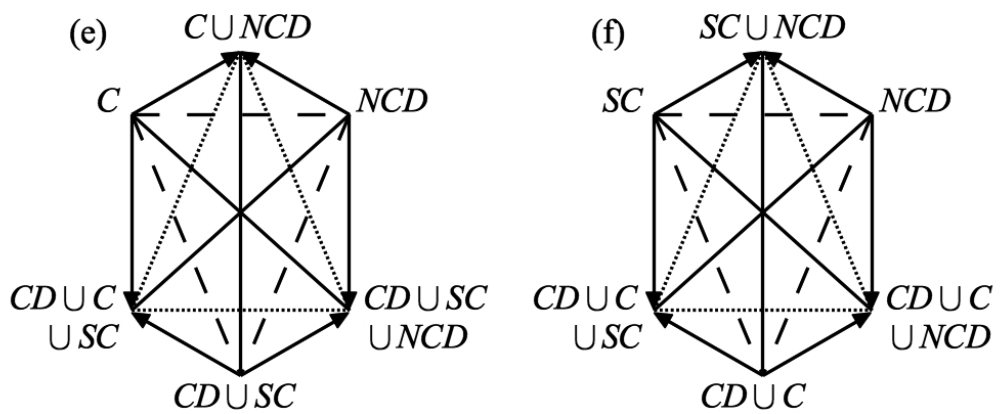

The impossibility of $C D(\varphi, \varphi)$-i.e. the fact that $C D \cap \Delta=\emptyset$ - has an important consequence for the the type of Aristotelian diagram that we are dealing with: even though the original JSB hexagon in Figure 10 is a weak one, the new JSB hexagon in Figure 11 is strong. After all, although $C \cup S C \cup N C D$ is not the top element of $\wp^{\cup}(\mathcal{O G})$, the fact that $C D \cap \Delta=\emptyset$ entails that $(C \cap \Delta) \cup(S C \cap \Delta) \cup(N C D \cap \Delta)=(C \cup S C \cup N C D) \cap \Delta$ is indeed the top element of $\wp^{\cup}(\{R \cap \Delta \mid R \in \mathcal{O G}\})$. Furthermore, we also find that 
Figure 10. A weak Jacoby-Sesmat-Blanché hexagon inside the Aristotelian $\mathrm{RDH}$ for $\wp(\mathcal{O G})$, shown in terms of (a) relations and (b) statements

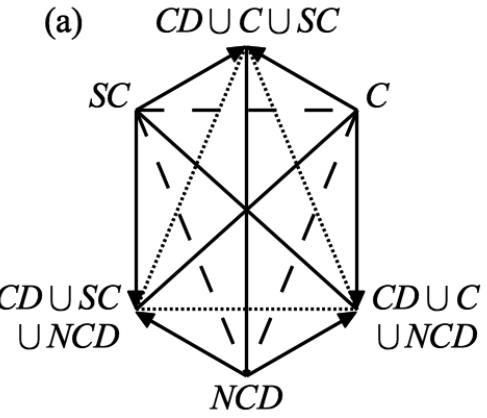

$$
\begin{array}{ll}
(\varphi, \varphi) \in C D \cup S C \cup N C D & \text { iff } S C(\varphi, \varphi) \text { or } N C D(\varphi, \varphi) \\
& \text { iff } \varphi \text { is a tautology or } \varphi \text { is a contingency } \\
& \text { iff } \varphi \text { is satisfiable, } \\
(\varphi, \varphi) \in C D \cup C \cup N C D \quad & \text { iff } C(\varphi, \varphi) \text { or } N C D(\varphi, \varphi) \\
& \text { iff } \varphi \text { is a contradiction or } \varphi \text { is a contingency } \\
& \text { iff } \varphi \text { is a not a tautology, } \\
(\varphi, \varphi) \in C D \cup C \cup S C & \text { iff } C(\varphi, \varphi) \text { or } S C(\varphi, \varphi) \\
& \text { iff } \varphi \text { is a contradiction or } \varphi \text { is a tautology } \\
& \text { iff } \varphi \text { is a not a contingency. }
\end{array}
$$

By filling in the same formula twice, the six relations in the original JSB hexagon in Figure 10 thus turn out to correspond to some well-known metalogical notions, such as being a tautology, being satisfiable, being a contradiction and being contingent. At this point, it might be objected that two of the six relations do not correspond to a 'primitive' metalogical notion: $(C D \cup C \cup N C D) \cap \Delta$ and $(C D \cup C \cup S C) \cap \Delta$ can only be 'negatively described', as not being a tautology and not being a contingency, respectively. However, this discrepancy in lexicalization is entirely to be expected, since it is perfectly in line with previous, empirical work on natural language decorations of the classical square and strong JSB hexagon. Linguists have found exactly the same discrepancy in various closed lexical fields, such as the quantifiers and the temporal adverbs. ${ }^{12}$ The correspondence between $\wp^{\cup}(\{R \cap \Delta \mid R \in \mathcal{O G}\})$ and the lexical field of tautology and related metalogical notions shows that this partial lexicalization pattern arises not only in natural languages, but even in metalogical jargon.

\footnotetext{
${ }^{12}$ Using the familiar $\mathrm{A} / \mathrm{I} / \mathrm{E} / \mathrm{O}$ abbreviations for the elements of the square, and writing $\mathrm{U}$ and $\mathrm{Y}$ for the hexagon's uppermost and lowermost elements, the non-lexicalized elements are exactly $\mathrm{O}$ and $\mathrm{U}$. A pragmatic (neo-Gricean) explanation for the non-lexicalization of the O-element in the square has been developed in [38, 39], and later extended to also account for the non-lexicalization of the U-element in the JSB hexagon [46, 73].
} 
Figure 11. (a) A strong Jacoby-Sesmat-Blanché hexagon for statements of the form $R(\varphi, \varphi)$ (with $R \in \wp^{\cup}(\mathcal{O G})$ ), and

(b) its reformulation using more familiar terminology

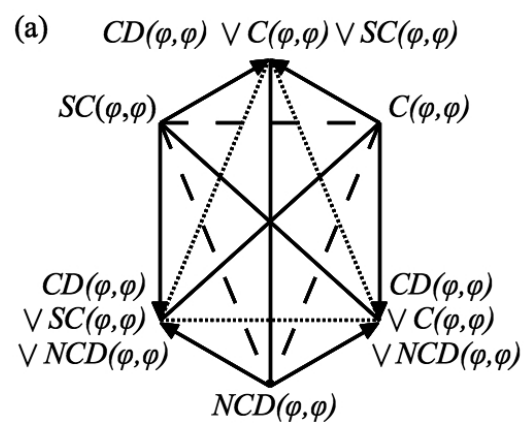

(b)

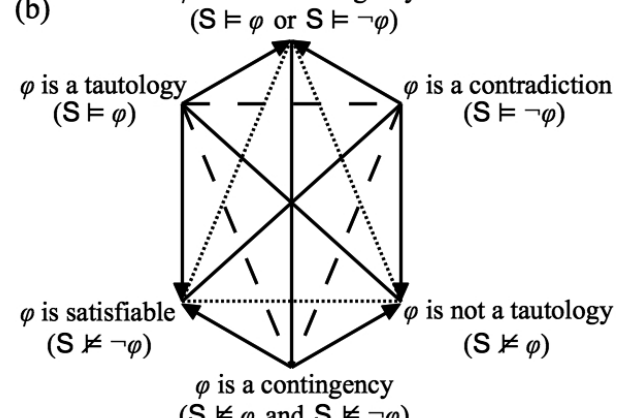

$(S \not \models \varphi$ and $S \not \nvdash \neg \varphi)$

More importantly, however, because of this correspondence, the JSB hexagon in Figure 11(a) can be reformulated as the more familiar JSB hexagon in Figure 11(b). This metalogical hexagon was first studied by Béziau in [4, Paragraph 3.2.4] and [5], and later also by Diaconescu [33]. What we have shown here is that this hexagon can be seen as (a special instance of) a subdiagram of the Aristotelian $\mathrm{RDH}$ for $\mathcal{O G}$ that was introduced in the previous subsection.

Finally, it should be emphasized that the construction of Aristotelian diagrams for metalogical notions such as tautology and satisfiability crucially depends on the assumption that the underlying logical system $\mathrm{S}$ be consistent. To illustrate this, we will ignore (non-)contingency, and thus focus on the Aristotelian square in Figure 12(a) (which can be seen as a subdiagram of the JSB hexagon in Figure 11). The contrariety, the subcontrariety and the two subalternations in this square only hold if $S$ is assumed to be consistent. For example, if $S$ is not consistent, i.e. if there are no S-models, then we simultaneously have $S \models \varphi$ and $S \models \neg \varphi$, and thus lose the contrariety between $\varphi$ being a tautology and $\varphi$ being a contradiction. Similar remarks apply to the other Aristotelian relations in the square (except for the two contradictions). In sum, then, without the assumption that $\mathrm{S}$ be consistent, the metalogical square in Figure 12(a) 'degenerates' into the Aristotelian 'cross' in Figure 12(b).

\subsection{Aristotelian Hexagons for Strong and Weak (Sub)contrariety}

Throughout the history of philosophical logic, the relations of contrariety and subcontrariety have been defined in two related, but subtly different ways. The resulting notions can be called strong and weak (sub)contrariety. Working in a logical system $\mathrm{S},{ }^{13}$ the definitions look as follows:

13 Recall that in Section 2, we distinguished at least three ways of defining the Aristotelian relations, viz. Definitions 2.1, 2.2 and 2.3. The distinction between strong and weak 
Figure 12. (a) A classical Aristotelian square for metalogical notions, on the assumption that $\mathrm{S}$ be consistent, (b) a degenerated Aristotelian 'cross' for the same metalogical notions, without the assumption that $\mathrm{S}$ be consistent

(a)

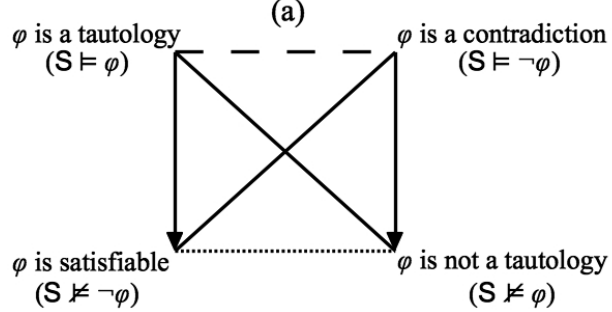

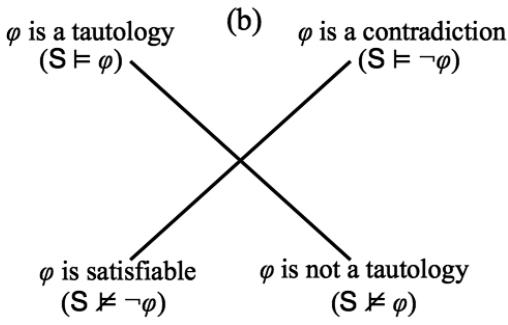

Definition 4.1. Two formulas $\varphi, \psi \in \mathcal{L}_{\mathrm{S}}$ are said to be

$\begin{array}{llll}\text { strongly S-contrary } & \text { iff } \mathrm{S} \models \neg(\varphi \wedge \psi) & \text { and } & \mathrm{S} \not \varphi \vee \psi, \\ \text { weakly S-contrary } & \text { iff } \mathrm{S} \models \neg(\varphi \wedge \psi), & \\ \text { strongly S-subcontrary } & \text { iff } \mathrm{S} \not \models \neg(\varphi \wedge \psi) & \text { and } & \mathrm{S} \models \varphi \vee \psi, \\ \text { weakly S-subcontrary } & \text { iff } & & \mathrm{S} \models \varphi \vee \psi .\end{array}$

The strong notions of (sub)contrariety are defined in terms of a $\models$ condition and $a \mid \neq$-condition; the corresponding weak notions keep the former, but discard the latter. Note that the notions of (sub)contrariety defined in Sections 2 and 3 are the strong ones (also see Footnote 13). Other uses of the strong notions can be found in $[62,74,79]$. In contrast, the weak notions of (sub)contrariety are used in $[14,57,71]$. In recent years, the distinction itself has become the topic of some discussion. For example, Humberstone [42] links the strong and weak notions of (sub)contrariety to "traditionalist" and "modernist" approaches to logic, while Demey and Smessaert [27] show that the relation between the two notions can be understood in terms of Gricean pragmatics. Finally, and most relevant for our current purposes, Béziau [4, Paragraph 4.1.2] has used the strong and weak notions of (sub)contrariety to define a metalogical decoration for an Aristotelian hexagon.

Because of their definition, the strong notions of contrariety and subcontrariety trivially belong to $\mathcal{O G}$ (recall Definition 3.1). However, the corresponding weak notions can also be expressed in terms of opposition relations:

$$
\begin{array}{rll}
(\varphi, \psi) \in C D \cup C \text { iff } & C D(\varphi, \psi) \text { or } C(\varphi, \psi) \\
& \text { iff } \quad(S \models \neg(\varphi \wedge \psi) \text { and } S \models \varphi \vee \psi) \text { or } \\
& (S \models \neg(\varphi \wedge \psi) \text { and } S \mid \models \varphi \vee \psi) \\
\text { iff } S \models \neg(\varphi \wedge \psi) & \\
\text { iff } \quad \varphi \text { and } \psi \text { are weakly S-contrary, }
\end{array}
$$

(sub)contrariety can be made in each of these approaches. We choose to work in the line of Definition 2.2 here, because the distinction between strong and weak (sub)contrariety only plays a role at the object-logical level. (At the metalogical level, we exclusively work with the strong notions of (sub)contrariety throughout this paper.) 
and analogously, $(\varphi, \psi) \in C D \cup S C$ iff $\varphi$ and $\psi$ are weakly S-subcontrary. Using ' $s$ '- and ' $w$ '-subscripts for 'strong' and 'weak', respectively, we thus have:

$$
\begin{array}{rrrrr}
C_{s} & = & C, & S C_{s}= & S C, \\
C_{w}= & C D \cup C, & S C_{w}= & C D \cup S C .
\end{array}
$$

The strong and weak notions of (sub)contrariety thus all belong to $\wp^{\cup}(\mathcal{O G})$, and hence occur in (subdiagrams of) the Aristotelian RDH that was constructed in Subsection 4.1. Consider, for example, the strong JSB hexagon that was already shown in Figure 9(a), but is repeated here (modulo some rotations and reflections) as Figure 13(a), and reformulated using the strong/weak terminology as Figure 13(b). This hexagon visualizes the Aristotelian relations holding between the notions of contradiction, strong contrariety and weak contrariety (and their negations). ${ }^{14}$ It can also be used to shed some new light on the interesting question why an otherwise wellregulated piece of scientific jargon such as the term contrary can come to be ambiguous.

The point is that the term contrary is ambiguous in a highly systematic fashion: it has a weak interpretation (which is compatible with contradiction) and a strong interpretation (which is incompatible with contradiction). A strong JSB hexagon such as the one shown in Figure 13(c) has been used in $[73$, p. 624] to explain why the natural language expression some is ambiguous between a unilateral interpretation some $_{1}$ (which is compatible with all, i.e. at least one) and a bilateral interpretation some s $_{2}$ (which is incompatible with all, i.e. some but not all). ${ }^{15}$ The precise linguistic-cognitive details of this explanation need not concern us here, but given the striking analogy between all/some $1 /$ some $_{2}$ and $C D / C_{w} / C_{s}$, it should not be surprising if a broadly similar account also applies to the latter.

\subsection{An Aristotelian Octagon for Strong and Weak (Sub)contrariety}

In the previous subsection, we constructed a strong JSB hexagon for strong and weak contrariety (see Figure 9(a) and Figure 13), and another strong JSB hexagon for strong and weak subcontrariety (see Figure 9(b) and Footnote 14). So far, however, we have not yet considered the interaction between these two (pairs of) notions. The key insight in studying this interaction is that strong contrariety and strong subcontrariety are themselves contrary

14 Completely analogously, one can of course also construct a strong JSB hexagon for strong/weak subcontrariety instead of strong/weak contrariety; see Figure 9(b).

${ }^{15}$ The distinction between unilateral and bilateral interpretations can also be made for other quantifiers, such as many and few [80, p. 484ff.], and even for richer, non-closed lexical fields [73, p. 640ff.]. For example, by replacing all and some by resp. human and animal in Figure 13(c), one can explain the ambiguity of the word animal, which has a biological interpretation (which is compatible with human) as well as a more 'everyday life' interpretation (which is incompatible with human). Extensive linguistic research has shown that systematic ambiguities such as these show up across a wide range of natural languages. 
Figure 13. (a) A strong JSB hexagon with elements of $\wp^{\cup}(\mathcal{O G})$, (b) its reformulation in terms of weak and strong contrariety, and (c) an analogous strong JSB hexagon for the unilateral and bilateral interpretations of the natural language quantifier some
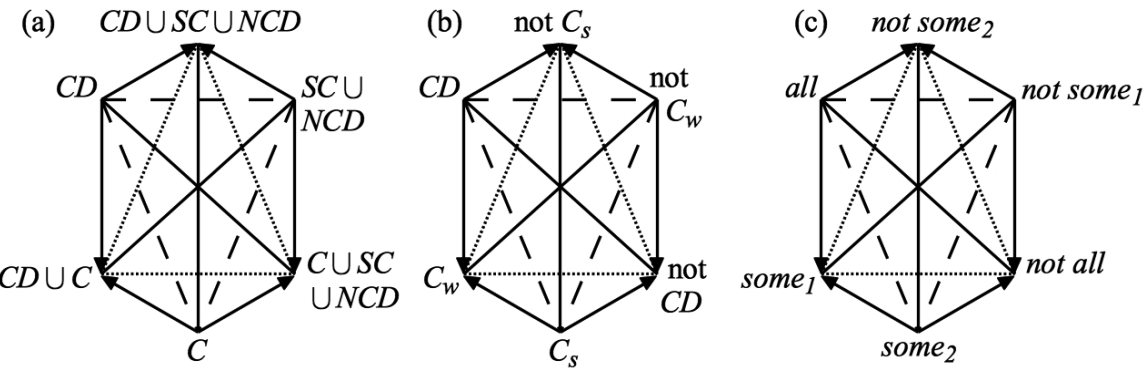

FiguRE 14. (a) Aristotelian square with elements of $\wp^{\cup}(\mathcal{O G})$, (b) its reformulation in terms of strong (sub)contrariety

(a)

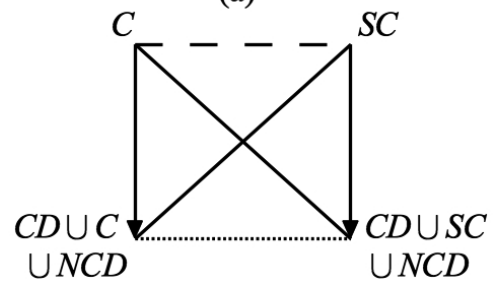

(b)

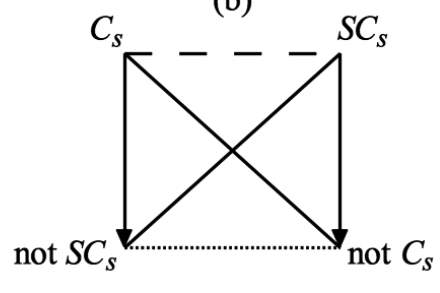

to each other. ${ }^{16}$ After all, as was already said in Section 2, it holds that $C \cap S C=\emptyset$ and $C \cup S C \neq \mathbb{B}(\mathrm{S}) \times \mathbb{B}(\mathrm{S})$, i.e. $C \cap S C$ is the bottom element, but $C \cup S C$ is not the top element of the Boolean algebra $\wp^{\cup}(\mathcal{O G})$. Consequently, strong contrariety and strong subcontrariety can be used to construct a classical Aristotelian square, which is shown in Figure 14.

In order to integrate the weak notions of (sub)contrariety into this square, we need to 'decompose' its subalternations. First of all, recalling that weak contrariety is $C_{w}=C D \cup C$, we can add $C_{w}$ to the square by decomposing its left subalternation into $C \rightarrow C D \cup C$ and $C D \cup C \rightarrow C D \cup C \cup N C D$. In order to keep the resulting diagram closed under contradiction, we also add the $\wp^{\cup}(\mathcal{O G})$-complement of $C D \cup C$, viz. $S C \cup N C D$, by decomposing the square's right subalternation into $S C \rightarrow S C \cup N C D$ and $S C \cup N C D \rightarrow$

\footnotetext{
${ }^{16}$ To re-emphasize a point that was already made earlier (see Footnote 13): note that in saying that strong contrariety and strong subcontrariety are themselves contrary, we are using the notion of contrariety (in italics) at the metalogical level, and are thus making use of the strong notion of contrariety. To put it more explicitly: strong contrariety and strong subcontrariety are themselves strongly contrary.
} 
Figure 15. Two Sherwood-Czezowski hexagons that result from adding (a) weak contrariety $(C D \cup C)$ and (b) weak subcontrariety $(C D \cup S C)$ to the square in Figure 14

(a)

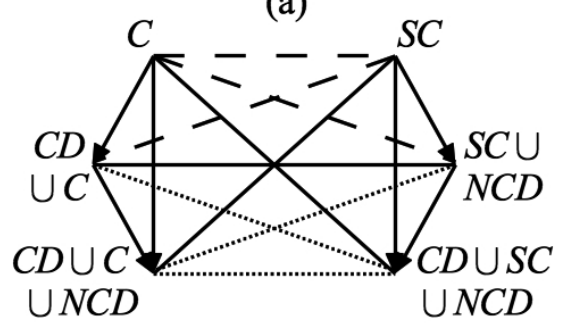

(b)

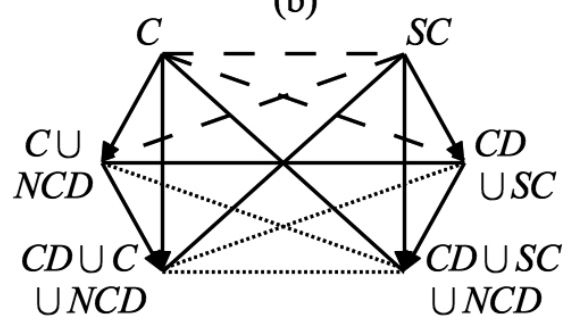

$C D \cup S C \cup N C D$. The resulting Aristotelian diagram is a Sherwood-Czeżowski hexagon, which is shown in Figure 15(a). ${ }^{17}$ Secondly, recalling that weak subcontrariety is $S C_{w}=C D \cup S C$, we can add weak subcontrariety in an entirely analogous fashion, and will thereby obtain a second Sherwood-Czeżowski hexagon, which is shown in Figure 15(b).

These two Sherwood-Czeżowski hexagons can be combined into a single Aristotelian diagram, viz. a Buridan octagon, which is shown in Figure $16(\mathrm{a}) .{ }^{18}$ Note that the four relations in the 'middle' of this octagon (i.e. those that do not occur in the square in Figure 14) do not stand in any Aristotelian relation at all (except for the obvious contradictions between $C D \cup C$ and $S C \cup N C D$, and between $C \cup N C D$ and $C D \cup S C$ ). Consider, for example, the relations $C D \cup C$ and $C \cup N C D$ :

- since $(C D \cup C) \cap(C \cup N C D)=C \neq \emptyset$, it follows that $C D \cup C$ and $C \cup N C D$ are neither contradictory nor contrary,

- since $(C D \cup C) \cup(C \cup N C D)=C D \cup C \cup N C D \neq \mathbb{B}(\mathrm{S}) \times \mathbb{B}(\mathrm{S})$, it follows that $C D \cup C$ and $C \cup N C D$ are not subcontrary,

- since $(C D \cup C) \cap(C \cup N C D)=C \neq C D \cup C$, it follows that there is no subalternation from $C D \cup C$ to $C \cup N C D$,

- since $(C \cup N C D) \cap(C D \cup C)=C \neq C \cup N C D$, it follows that there is no subalternation from $C \cup N C D$ to $C D \cup C$.

Recalling that the Buridan octagon in Figure 16(a) is a subdiagram of the Aristotelian $\mathrm{RDH}$ for $\wp^{\cup}(\mathcal{O G})$ that was described in Subsection 4.1, it should be noted that this Buridan octagon consists of exactly those relations

\footnotetext{
${ }^{17}$ The Sherwood-Czeżowski hexagons are so-called because they were long thought to have first been studied in the 1950s by Czeżowski [19], but it has recently been argued that they were already used by the 13th-century logician William of Sherwood [48, 49].

${ }^{18}$ The Buridan octagons are so-called because they were first studied by the 14th-century logician John Buridan [40,67]. Buridan octagons that can be embedded as Aristotelian subdiagrams inside an Aristotelian RDH, have recently also been called 'rhombicubes', based on the cube-like shape with two rhombic faces of this embedding [30, 32, 78, 80, 81]. The term 'rhombicube' was introduced recently, and derives from the fact that if we consider a Buridan octagon as an Aristotelian subdiagram embedded inside the Aristotelian $\mathrm{RDH}$, it has a cube-like shape with two rhombic faces.
} 
Figure 16. (a) Buridan octagon with elements of $\wp^{\cup}(\mathcal{O G})$, (b) reformulation in terms of strong/weak (sub)contrariety

(a)

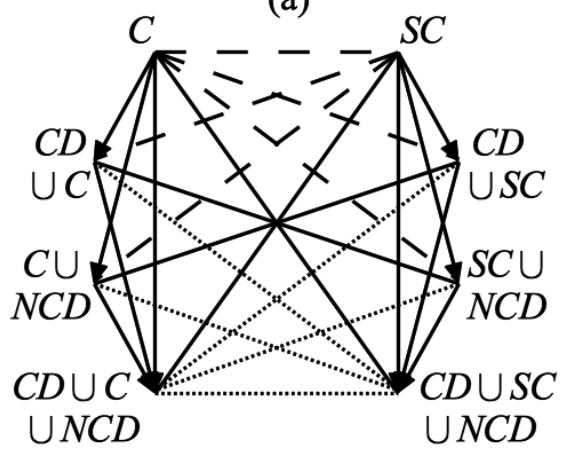

(b)

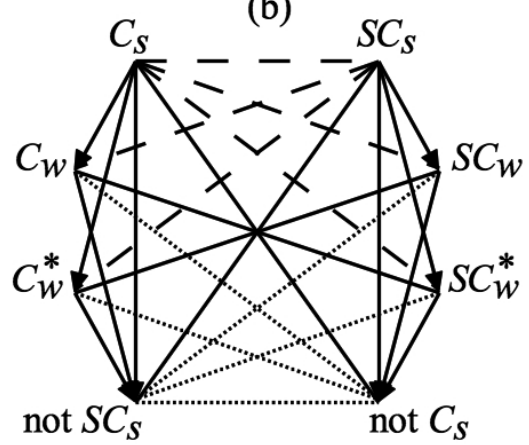

$R \in \wp^{\cup}(\mathcal{O G})$ that contain either $C$ or $S C$ but not both, i.e. such that $C \subseteq R$ iff $S C \nsubseteq R$. We can also construct the 'complement' of this Buridan octagon, which contains exactly those relations $R \in \wp^{\cup}(\mathcal{O G})$ such that $C \subseteq R$ iff $S C \subseteq R$. It is well-known that the complement of a Buridan octagon is itself a strong JSB hexagon $[78,81],{ }^{19}$ and hence, this complement is a strong JSB hexagon inside the Aristotelian RDH for $\wp^{\cup}(\mathcal{O G})$, viz. the one shown in Figure 9(c).

Finally, and perhaps more importantly for our current purposes, the Buridan octagon in Figure 16(a) also suggests that there is not just one, but actually two ways in which the strong notion of (sub)contrariety can be weakened. Recall that the strong notion of contrariety $\left(C_{s}=C\right)$ is defined in terms of a $\models$-condition and a $\not \models$-condition. As was discussed in the previous subsection, several authors have proposed a weaker version of contrariety by dropping the $\not \forall$-condition, thereby obtaining $C_{w}=C D \cup C$. The Buridan octagon suggests, however, that there is also an alternative notion of weak contrariety, viz. $C_{w}^{*}=C \cup N C D$. It is easy to see that this alternative notion of weak contrariety is 'dual' to the original one, in the sense that it can be obtained from strong contrariety by dropping the $=$-condition instead of the $\forall$-condition. Looking again at Figure 16(a), we cannot distinguish between the two weak notions of contrariety, since $C D \cup C$ and $C \cup N C D$ stand in exactly the same Aristotelian relations to the relations of the original square (i.e. $C, S C, C D \cup C \cup N C D$ and $C D \cup S C \cup N C D$ ):

- both are entailed by strong contrariety (i.e. $C$ ),

- both entail the absence of strong subcontrariety (i.e. $C D \cup C \cup N C D$ ),

- both are contrary to strong subcontrariety (i.e. $S C$ ),

${ }^{19}$ There are 8 relations $R \in \wp^{\cup}(\mathcal{O G})$ such that $C \subseteq R$ iff $S C \subseteq R$, but two of them are $\wp^{\cup}(\mathcal{O G})$ 's bottom element $\emptyset$ (for which it holds that $C \nsubseteq \emptyset$ and $S C \nsubseteq \emptyset$ ) and its top element $\mathbb{B}(\mathrm{S}) \times \mathbb{B}(\mathrm{S})$ (for which it holds that $C \subseteq \mathbb{B}(\mathrm{S}) \times \mathbb{B}(\mathrm{S})$ and $S C \subseteq \mathbb{B}(\mathrm{S}) \times \mathbb{B}(\mathrm{S})$ ), and these do not occur inside any Aristotelian diagram (recall Footnote 9). 
FiguRE 17. (a) Béziau's partially correct hexagon, (b) a plausible reformulation in terms of elements of $\wp^{\cup}(\mathcal{O G})$, and

(c) the corrected version
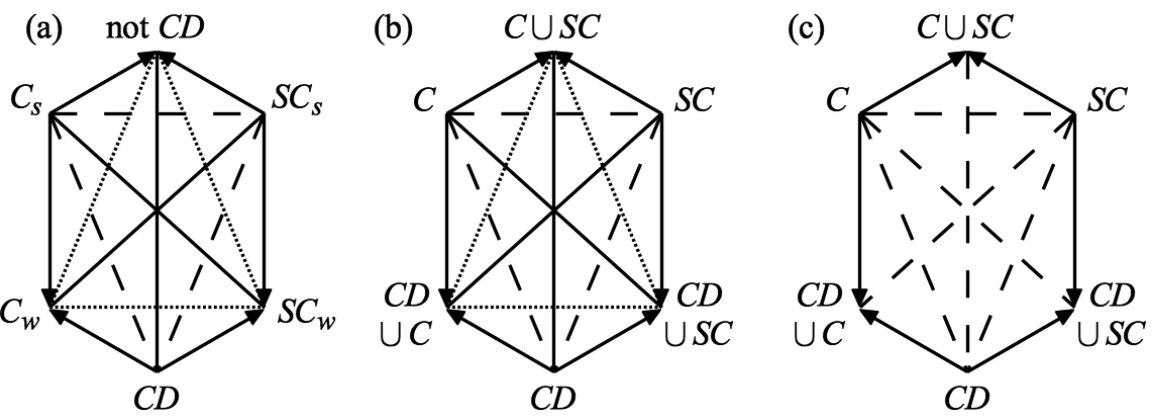

- and finally, both are subcontrary to the absence of strong contrariety (i.e. $C D \cup S C \cup N C D$ ).

Similar remarks can be made, of course, for subcontrariety: in addition to strong subcontrariety $\left(S C_{s}=S C\right)$ and the original notion of weak subcontrariety $\left(S C_{w}=C D \cup S C\right)$, we also get a new notion of weak subcontrariety $\left(S C_{w}^{*}=S C \cup N C D\right)$ that is 'dual' to the original one. Using this new terminology, the relations in the Buridan octagon in Figure 16(a) can be reformulated more evocatively as in Figure 16(b).

\subsection{Correcting Béziau's Hexagon for Strong and Weak (Sub)contrariety}

In the previous two subsections, we have constructed Aristotelian diagrams for the strong and weak notions of contrariety, and their interaction. In each of these diagrams, the relation of contradiction played an important role, in the sense that $C D$ is a subset of exactly half of the relations at its vertices (for example, in Buridan octagon in Figure 16, it holds that $C D \subseteq C_{w}, S C_{w}$, not $C_{s}$, not $\left.C_{s}\right)$. However, none of these diagrams contains $C D$ 'by itself'. Béziau [4, Paragraph 4.1.2] has studied the interaction between $C D$ and strong/weak (subcontrariety), and claims that this interaction can be visualized by means of a JSB hexagon [4, Figure 40], which is shown here as Figure 17(a). Note that Béziau [4, p. 30] explicitly defines the notions of strong (sub)contrariety and weak (sub)contrariety, and his definition corresponds exactly to our Definition 4.1. However, Béziau does not state explicitly how he understands the relation labeled as 'not $C D$ ' in his hexagon; we will return to this missing definition very soon (see Footnote 21).

Upon closer examination, Béziau's hexagon turns out to be only partially correct; more specifically, we find:

- the three contrarieties and the six subalternations are correct,

- the three contradictions are incorrect,

for example, $C_{s}$ and $S C_{w}$ are said to be contradictory, but since $C_{s} \cap$ $S C_{w}=C \cap(C D \cup S C)=\emptyset$ and $C_{s} \cup S C_{w}=C \cup(C D \cup S C)=$ 
$C D \cup C \cup S C \neq \mathbb{B}(\mathrm{S}) \times \mathbb{B}(\mathrm{S})$, these relations are contrary, rather than contradictory,

- the three subcontrarieties are incorrect,

for example, $C_{w}$ and $S C_{w}$ are said to be subcontrary, but since $C_{w} \cup$ $S C_{w}=(C D \cup C) \cup(C D \cup S C)=C D \cup C \cup S C \neq \mathbb{B}(\mathrm{S}) \times \mathbb{B}(\mathrm{S})$, these relations are not subcontrary; in fact, it can be shown that they do not stand in any Aristotelian relation at all.

The contradictions in Béziau's hexagon in Figure 17(a-b) should thus be replaced with contrarieties, and similarly, the subcontrarieties should be deleted (and not replaced with any Aristotelian relation at all). Figure 17(c) shows the Aristotelian diagram that is the result of making these corrections to Béziau's original hexagon. It should be emphasized that this corrected hexagon is highly exotic, since it does not obey the nearly universally accepted principle that Aristotelian diagrams should consist of pairs of contradictory formulas. ${ }^{20}$

Based on the observations made above, a uniform explanation can be given for the errors in the hexagon in Figure 17(a-b): Béziau seems to have ignored the relation $N C D$. After all, the incorrect contradictions and subcontrarieties are based on the assumption that the universal relation over $\mathbb{B}(\mathrm{S})$ is $C D \cup C \cup S C$, whereas actually it is $C D \cup C \cup S C \cup N C D .^{21}$ Putting it in less abstract terms, Béziau seems to have ignored the fact that there exist pairs of formulas - e.g. the pairs $(p, q)$ and $(p, p)$ in CPL - that are not contradictory, not contrary, and not subcontrary to each other (all we can say is that $N C D_{\mathrm{CPL}}(p, q)$ and $\left.N C D_{\mathrm{CPL}}(p, p)\right) .^{22}$

A correct Aristotelian diagram for the interaction between $C D$ and strong/weak subcontrariety can be obtained by considering the complementclosure of Béziau's hexagon, i.e. the Aristotelian diagram that is obtained by adding the complements of all relations that occur in the original diagram. ${ }^{23}$ The resulting diagram is a dodecagon, which is shown in Figure 18.

\footnotetext{
${ }^{20}$ The systematic study of such non-standard Aristotelian diagrams is still in its infancy; some preliminary results can be found in [60,61].

21 Whether this explanation also applies to the contradiction between $C D$ and 'not $C D$ ', depends on how the latter relation is interpreted (recall that Béziau does not explicitly say what he means by 'not $C D$ '). If 'not $C D$ ' is interpreted as $C \cup S C \cup N C D$, then $C D$ and 'not $C D$ ' are indeed contradictory to each other, and this relation in Béziau's hexagon in Figure 17(a) is correct after all. However, given the uniform explanation for the two other contradictions and the three subcontrarieties, it seems far more likely that Béziau means 'not $C D$ ' to be interpreted as $C \cup S C$ - this interpretation is also assumed in Figure 17(b) , and in that case, it is incorrect to say that $C D$ and 'not $C D$ ' are contradictory to each other (since $C D \cup(C \cup S C) \neq \mathbb{B}(\mathrm{S}) \times \mathbb{B}(\mathrm{S})$ ).

${ }^{22}$ Using Pellissier's [64] terminology, yet another reformulation might look as follows: Béziau takes the hexagon in Figure 17 to be a strong JSB hexagon, but actually it is a weak JSB hexagon. However, this reformulation has to be taken with a grain of salt: Béziau's hexagon is incorrect in its contradictions and subcontrarieties, so it is not a proper JSB hexagon to begin with, and thus the question whether it is a strong or a weak one does not even arise, strictly speaking.

${ }^{23}$ For most Aristotelian diagrams, this operation does not make much sense, since they are already closed under negation, and are thus their own complement-closure.
} 
Figure 18. (a) The complement-closure of Béziau's hexagon, (b) its reformulation in terms of strong/weak/weak* (sub)contrariety
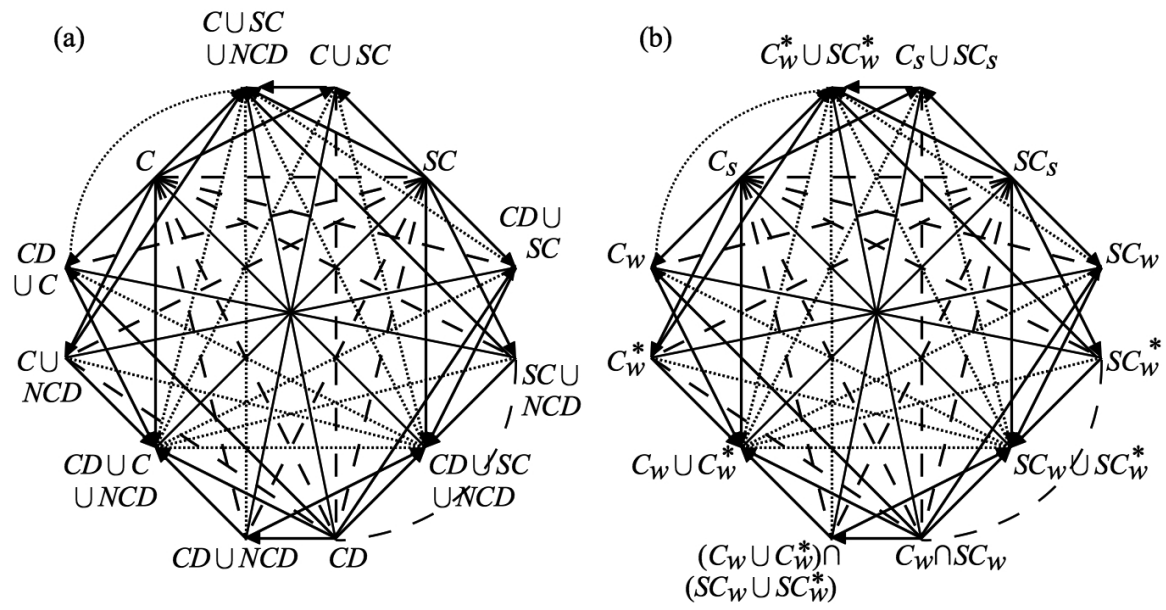

This dodecagon contains all the other Aristotelian diagrams shown in Subsections 4.3-4.5 as subdiagrams. Comparing this dodecagon to the Aristotelian $\mathrm{RDH}$ that was described in Subsection 4.1, we see that it lacks only two relations, namely precisely those that were originally forgotten by Béziau: $N C D$ and its contradictory, $C D \cup C \cup S C$.

One can also adopt an alternative perspective on the relationship between Béziau's hexagon in Figure 17(a) and the Aristotelian RDH for $\mathcal{O G}$ in Figure 8(b). Recall that $\wp^{\cup}(\mathcal{O G})$ consists of 16 relations, which are of the form $\bigcup \mathcal{X}$, for $\mathcal{X} \subseteq \mathcal{O G}$. Now, if $N C D=\emptyset$, then these 16 relations collapse pairwise into 8 relations; for example, $C D \cup N C D=C D \cup \emptyset=C D$. The table below describes all these collapses:

\begin{tabular}{|c|c|c|c|c|c|}
\hline $\mathrm{RDH}$ & & collapse & collapse & & $\mathrm{RDH}$ \\
\hline$C D$ & $\rightarrow$ & $C D$ & $C \cup S C$ & $\leftarrow$ & $C \cup S C \cup N C D$ \\
\hline$C D \cup N C D$ & $\lambda$ & & & к & $C \cup S C$ \\
\hline$C$ & $\rightarrow$ & $C$ & $C D \cup S C$ & $\leftarrow$ & $C D \cup S C \cup N C D$ \\
\hline$C \cup N C D$ & $\lambda$ & & & $\kappa$ & $C D \cup S C$ \\
\hline$S C$ & $\rightarrow$ & $S C$ & $C D \cup C$ & & $C D \cup C \cup N C D$ \\
\hline$S C \cup N C D$ & $>$ & & & $\kappa$ & $C D \cup C$ \\
\hline$N C D$ & $\rightarrow$ & {$[\emptyset]$} & {$[C D \cup C \cup S C]$} & & $C D \cup C \cup S C$ \\
\hline$[\emptyset]$ & $\lambda$ & & & K & {$[C D \cup C \cup S C \cup N C D]$} \\
\hline
\end{tabular}

In this table, square brackets indicate that a relation is the top- or bottom element in its respective Boolean algebra, and is thus not included in Aristotelian diagrams (recall Footnote 9). We thus see that the $16-2=14$ relations of the Aristotelian $\mathrm{RDH}$ for $\mathcal{O G}$ pairwise collapse into the $\frac{16}{2}-2=6$ 
relations of Béziau's hexagon. ${ }^{24}$ In other words, Béziau's hexagon is correct after all (and plays a role analogous to our $\mathrm{RDH}$ ), if only we are prepared to make the assumption that $N C D=\emptyset$. This leads to a more charitable interpretation of Béziau's hexagon, since the only substantial criticism that can now be made is that he should have stated more explicitly that he was working under the assumption that $N C D=\emptyset$. The problem with this interpretation, however, is that the assumption that $N C D=\emptyset$ is itself extremely unrealistic. For example, in any consistent logical system $\mathrm{S}$, if $\varphi$ is S-contingent, it holds that $N C D_{\mathrm{S}}(\varphi, \varphi)$, and thus definitely $N C D_{\mathrm{S}} \neq \emptyset$. In all reasonable logical systems, it will thus be the case that $N C D \neq \emptyset$, which brings us back to the original conclusion that Béziau's hexagon in Figure 17 is partially incorrect, and should be replaced by the dodecagon in Figure 18 or even the full RDH in Figure 8(b).

We will finish this section with a more general remark. In recent years, Béziau has studied at least two metalogical decorations of Aristotelian diagrams, viz. a JSB hexagon for tautology, satisfiability and related notionssee Figure 11(b) - and a JSB hexagon for the interaction between contradiction and strong and weak notions of (sub)contrariety-see Figure 17(a). Until now, these metalogical hexagons appeared to be two 'independent' Aristotelian diagrams. In this section, however, we have shown that these hexagons are intimately related to each other, since both of them can be seen as (special instances of) subdiagrams of one and the same metalogical diagram, viz. the Aristotelian $\mathrm{RDH}$ for $\mathcal{O G}$ that was described in Subsection 4.1.

\section{Aristotelian Diagrams for the Implication Relations}

In this section we continue our study of metalogical decorations for Aristotelian diagrams, by considering various Aristotelian diagrams for the implication geometry $(\mathcal{I} \mathcal{G})$. Subsection 5.1 shows how all the diagrams for $\mathcal{O G}$ that were studied in Section 4 can systematically be turned into diagrams for $\mathcal{I} \mathcal{G}$. Next, Subsection 5.2 explores the connection between Aristotelian diagrams for $\mathcal{I} \mathcal{G}$ and Aristotelian diagrams for abstract ordering relations.

\subsection{From Opposition to Implication Decorations of Aristotelian Diagrams}

We have argued above that since $\mathcal{O G}$ is a partition of $\mathbb{B}(\mathrm{S}) \times \mathbb{B}(\mathrm{S})$-i.e. every pair of formulas stands in exactly one opposition relation-, the elements of $\wp^{\cup}(\mathcal{O G})$ can be used to decorate an Aristotelian RDH. Furthermore, all Aristotelian diagrams that were discussed in Section 4 can be seen as subdiagrams of this Aristotelian RDH. Now, one the one hand, $\mathcal{O G}$ and $\mathcal{I} \mathcal{G}$ are closely related to each other (recall Lemma 3.4), and on the other hand $\mathcal{I} \mathcal{G}$ is also a partition of $\mathbb{B}(S) \times \mathbb{B}(S)$ - i.e. every pair of formulas stands in exactly one implication relation (recall Lemma 3.5). Consequently, it should come

\footnotetext{
${ }^{24}$ Furthermore, note that on the assumption that $N C D=\emptyset$, Béziau's hexagon is a strong JSB hexagon. It is still conceptually very different, however, from the six strong JSB hexagons inside the Aristotelian RDH for $\mathcal{O G}$ that were listed in Figure 9, since the latter six do not depend on the assumption that $N C D=\emptyset$.
} 
as no surprise that the elements of $\wp^{\cup}(\mathcal{I} \mathcal{G})=\{\bigcup \mathcal{X} \mid \mathcal{X} \subseteq \mathcal{I} \mathcal{G}\}$ can also be used to decorate an Aristotelian $\mathrm{RDH}$, and furthermore, all the insights and Aristotelian diagrams for $\wp^{\cup}(\mathcal{O G})$ that were discussed in Section 4 can straightforwardly be transposed to $\wp^{\cup}(\mathcal{I} \mathcal{G})$.

We will now briefly describe some of these Aristotelian diagrams for $\mathcal{I G}$. First of all, in analogy to the weak JSB hexagon of opposition relations that was shown in Figure 10(a), we will consider the weak JSB hexagon of implication relations that is shown in Figure 19(a). In the light of Lemma 3.4, however, we will not fill in $(\varphi, \varphi)$ in these relations, but rather $(\varphi, \neg \varphi)$; the resulting metalogical JSB hexagon is shown in Figure 19(b). ${ }^{25}$ In terms of relations, this means that we are intersecting each relation $R \in \wp^{\cup}(\mathcal{I} \mathcal{G})$ with the relation $\nabla:=\left\{([\varphi],[\neg \varphi]) \mid \varphi \in \mathcal{L}_{\mathrm{S}}\right\}$ on $\mathbb{B}(\mathrm{S})$, and are thus no longer working in the Boolean algebra $\wp^{\cup}(\mathcal{I} \mathcal{G})$, but rather in the Boolean algebra $\wp^{\cup}(\{R \cap \nabla \mid R \in \mathcal{I} \mathcal{G}\})=\{(\bigcup \mathcal{X}) \cap \nabla \mid \mathcal{X} \subseteq \mathcal{I} \mathcal{G}\} .{ }^{26}$ Assuming that the underlying logical system $S$ is consistent, we find that

$\begin{array}{llll}(\varphi, \neg \varphi) & \in B I & \text { iff impossible, } \\ (\varphi, \neg \varphi) & \in L I & \text { iff } \varphi \text { is a contradiction, } \\ (\varphi, \neg \varphi) & \in R I & \text { iff } \varphi \text { is a tautology, } \\ (\varphi, \neg \varphi) & \in N I & \text { iff } \varphi \text { is a contingency, } \\ (\varphi, \neg \varphi) & \in B I \cup R I \cup N I & \text { iff } \varphi \text { is satisfiable, } \\ (\varphi, \neg \varphi) & \in B I \cup L I \cup N I & \text { iff } \varphi \text { is not a tautology, } \\ (\varphi, \neg \varphi) & \in B I \cup L I \cup R I & \text { iff } \varphi \text { is not a contingency. }\end{array}$

The metalogical hexagon in Figure 19(b) can thus be reformulated as the hexagon for tautology and related metalogical notions that was already shown in Figure 11(b). This well-known hexagon [4, 5, 33] can thus not only be seen as (a special instance) of a subdiagram of the Aristotelian $\mathrm{RDH}$ for $\mathcal{O G}$ (as was shown in Subsection 4.2), but also as (a special instance) of a subdiagram of the Aristotelian $\mathrm{RDH}$ for $\mathcal{I} \mathcal{G}$.

Another insight that can fruitfully be transposed from $\mathcal{O G}$ to $\mathcal{I G}$ is the distinction between strong and weak opposition relations. The notion of left-implication $(L I)$ that was defined in Definition 3.2 can be called strong (notation: $L I_{s}$ ), since it is defined in terms of a $\models$ - and a $\not \models$-condition. This definition can be weakened in two ways that are 'dual' to each other: $L I_{w}$ drops the $\not \models$-condition, while $L I_{w}^{*}$ drops the $=$-condition. ${ }^{27}$ Obviously, an

\footnotetext{
${ }^{25}$ It should be clear that we could also choose to systematically fill in $(\neg \varphi, \varphi)$ instead of $(\varphi, \neg \varphi)$ : by Lemma 3.4 the resulting JSB hexagon would be identical to the one shown in Figure 19(b), modulo symmetry over the hexagon's vertical axis - for example, $R I(\neg \varphi, \varphi)$ iff $\operatorname{LI}(\varphi, \neg \varphi)$.

${ }^{26}$ Recall that in Subsection 4.2, every relation $R \in \wp^{\cup}(\mathcal{O G})$ was intersected with the identity relation $\Delta=\left\{([\varphi],[\varphi]) \mid \varphi \in \mathcal{L}_{\mathcal{S}}\right\}$. It is easy to see that $\nabla=C D \in \mathcal{O G}$ and $\Delta=B I \in \mathcal{I G}$. Furthermore, in [79] it is shown that $C D$ and $B I$ are the most informative relations of $\mathcal{O G}$ and $\mathcal{I} \mathcal{G}$, respectively. The operations described here and in Subsection 4.2 are thus entirely analogous, since both of them involve intersecting the relations of one geometry with the most informative relation of the other geometry.

${ }^{27}$ The notion of entailment in contemporary logic is typically assumed to be reflexive $(\varphi \models \varphi)$, and thus does not correspond to $L I_{s}$, but rather to $L I_{w}$.
} 
Figure 19. (a) A weak Jacoby-Sesmat-Blanché hexagon decorated with elements of $\wp^{\cup}(\mathcal{I} \mathcal{G})$, (b) the result of filling in $(\varphi, \neg \varphi)$

(a) $\quad B I \cup L I \cup R I$

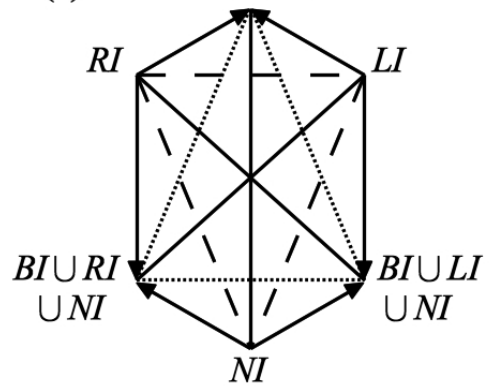

(b)

$$
B I(\varphi, \neg \varphi) \vee L I(\varphi, \neg \varphi)
$$

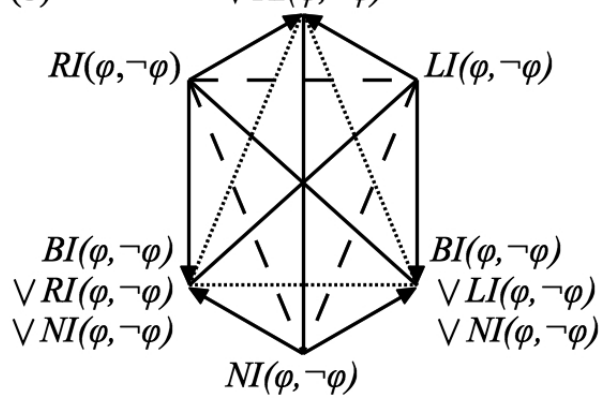

Figure 20. (a) Buridan octagon with elements of $\wp^{\cup}(\mathcal{I} \mathcal{G})$,

(b) reformulation in terms of strong/weak left- and rightimplication
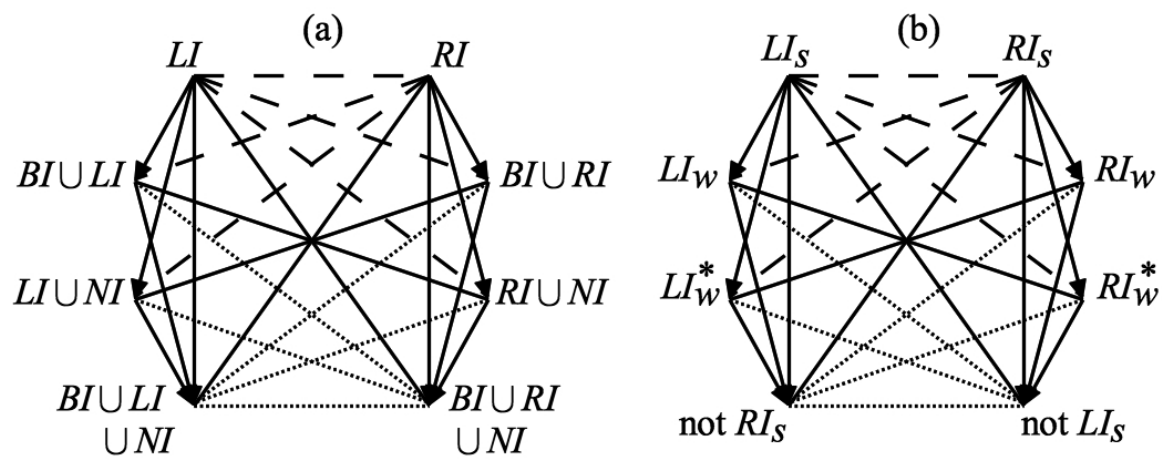

analogous distinction can be made for right-implication. All these relations are elements of $\wp^{\cup}(\mathcal{I} \mathcal{G})$ :
$L I_{s}=L I$,
$R I_{s}=R I$,
$L I_{w}=B I \cup L I$,
$R I_{w}=B I \cup R I$,
$L I_{w}^{*}=$
$L I \cup N I$,
$R I_{w}^{*}=$
$R I \cup N I$.

Together, these strong and weak notions of left- and right-implication can be used to decorate a Buridan octagon, which is shown in Figure 20.

\subsection{Aristotelian Diagrams for Ordering Relations}

In this subsection we will explore the relationship between the Aristotelian RDH for $\wp^{\cup}(I G)$ and Aristotelian diagrams for abstract ordering relations. The latter were among those that have sparked a renewed interested in Aristotelian diagrams since the middle of the 20th century [13], and have recently 
also been studied by Béziau [6]. Let's start by briefly recalling the definitions of partial and total ordering relations:

Definition 5.1. A binary relation $\leq$ on a set $S$ is said to be a partial order iff it satisfies the following conditions:

- for all $x \in S: x \leq x$,

- for all $x, y, z \in S$ : if $x \leq y$ and $y \leq z$, then $x \leq z$,

- for all $x, y \in S$ : if $x \leq y$ and $y \leq x$, then $x=y$.

(reflexivity)

(transitivity)

(antisymmetry)

Furthermore, $\leq$ is said to be a total order iff it satisfies all the conditions mentioned above, and additionally also the following one: ${ }^{28}$

- for all $x, y \in S: x \leq y$ or $y \leq x$.

(totality)

Finally, if $\leq$ is a partial or a total order, then the corresponding strict relation $<$ is defined as follows: $x<y: \Leftrightarrow(x \leq y$ and $x \neq y) .{ }^{29}$

Consider a total order $\leq$ on a set $S$. It follows straightforwardly from Definition 5.1 that the relations $\leq, \geq,<,>,=, \neq \in \wp(S \times S)$ can be used to decorate a JSB hexagon, which is shown in Figure 21(a). For example, $<$ and $>$ are contrary to each other, since there are no $x, y \in S$ such that simultaneously $x<y$ and $x>y$, but there are $x, y \in S$ such that neither $x<$ $y$ nor $x>y$ (for example, take $(x, y):=(x, x)$, for any $x \in S)$. Furthermore, the JSB hexagon for total orders is strong, since the union of the contrary relations $<,>$ and $=$ is the universal relation $S \times S$ (i.e. the top element of the Boolean algebra $\wp(S \times S))$ : it follows straightforwardly from the totality condition that for all $x, y \in S: x<y$ or $x>y$ or $x=y$. The fact that a total order can be used to decorate a strong JSB hexagon has long been known - in fact, it was already known in the 1950s by Sesmat [70] and Blanché $[11,12,13]$, two of the authors after which the JSB hexagon was originally named (recall Footnote 10).

If the relation $\leq$ is not assumed to be a total order, but merely a partial order, the Aristotelian hexagon in Figure 21(a) undergoes some changes:

- two of the three contradictions change into contrarieties, for example, $<$ and $\geq$ are contraries, since $<\cap \geq=\emptyset$ (i.e. there are no $x, y \in S$ such that simultaneously $x<y$ and $x \geq y$ ), and $<\cup \geq \neq S \times S$ (i.e. there are $x, y \in S$ such that neither $x<y$ nor $x \geq y$ )

- one of the three subcontrarieties is lost (and not replaced with any Aristotelian relation at all), viz. $\leq$ and $\geq$ are no longer subcontrary to each other, since $\leq \cup \geq$ $\neq S \times S$ (i.e. there are $x, y \in S$ such that neither $x \leq y$ nor $x \geq y$ ),

- the three contrarieties and the six subalternations remain unchanged.

\footnotetext{
${ }^{28}$ Note that if the totality axiom is added, the reflexivity axiom becomes redundant, since every relation that satisfies totality can easily be shown to also satisfy reflexivity.

${ }^{29}$ In case $\leq$ is a total order, the corresponding strict relation can also be defined as follows: $x<y: \Leftrightarrow \operatorname{not}(x \geq y)$. However, this alternative definition cannot be used if $\leq$ is only a partial order: if $\leq$ is a total order, then $\operatorname{not}(x \geq y)$ is equivalent to $(x \leq y$ and $x \neq y)$, but if $\leq$ is only a partial order, then these two statements are not equivalent to each other.
} 
FiguRE 21. Aristotelian hexagon for (a) a total order, and (b) a partial order

(a)

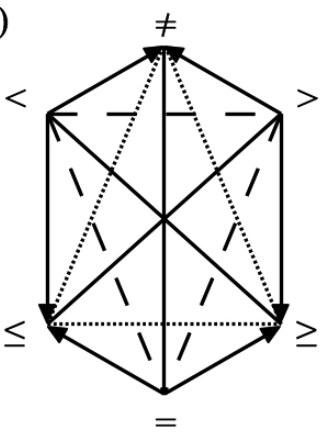

(b)

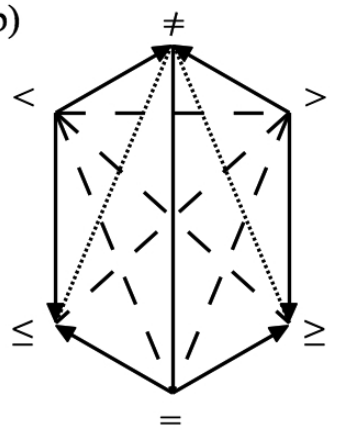

The resulting Aristotelian hexagon is shown in Figure 21(b), and was first studied in [60, Figure 59]. We would now like to point out a striking analogy between two conceptual processes that have been described in this section and the previous one:

- in Subsection 4.5: correcting Béziau's hexagon, i.e. from Figure $17(\mathrm{a}-\mathrm{b})$ to $17(\mathrm{c})$.

- just now: moving from a total order to a partial order, i.e. from Figure 21(a) to 21(b),

These two cases arise because of essentially one and the same reason. As was argued in Subsection 4.5, the first case shows that one cannot ignore the opposition relation of non-contradiction, i.e. one cannot assume (like Béziau seemed to do) that $N C D=\emptyset$. Completely analogously, the second case shows that in going from complete to partial orders (and thus dropping the totality axiom), one cannot ignore the relation of incomparability $\#:=\{(x, y) \in S \times S \mid \operatorname{not}(x \leq y)$ and $\operatorname{not}(x \geq y)\}=\{(x, y) \in$ $S \times S \mid \operatorname{not}(x=y)$ and $\operatorname{not}(x<y)$ and $\operatorname{not}(x>y)\}$, i.e. one cannot assume that $\#=\emptyset$. From this perspective, it is particularly ironic that Béziau explicitly states that his hexagon for contradiction and strong and weak (sub)contrariety in Figure 17(a) is "very similar to the ones presented by Sesmat and Blanché relating $<,>,=, \leq, \geq, \neq$ " $[4$, p. 30], and thus compares it to the hexagon for total orders in Figure 21(a), although it is actually much more similar to the hexagon for partial orders in Figure 21(b).

The Boolean closure of the hexagon for partial orders in Figure 21(b) is a Boolean algebra consisting of 16 elements, which - after leaving out the empty and universal relations (recall Footnote 9) - can be used to decorate an Aristotelian RDH. (A geometrical variant of this RDH can already be found in [60, Figure 59].) The atoms of this Boolean algebra are the relations $=,<,>$ and \#, and thus the Boolean algebra itself can be described as 
FIGURE 22. Aristotelian rhombic dodecahedron for partial orderings

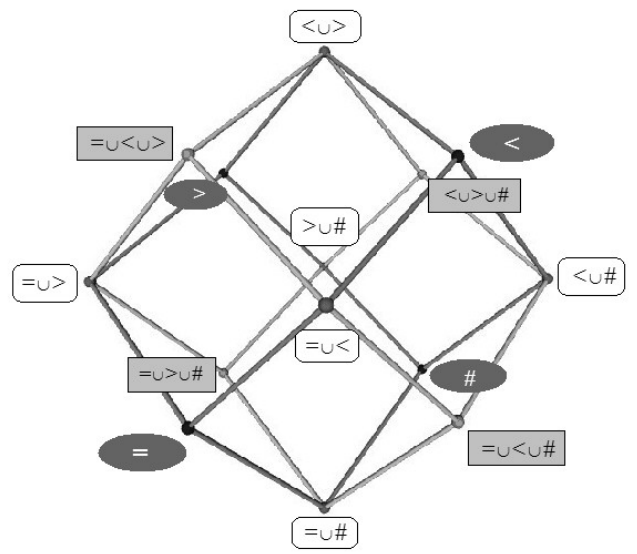

$\wp^{\cup}(\{=,<,>, \#\})=\{\bigcup \mathcal{X} \mid \mathcal{X} \subseteq\{=,<,>, \#\}\}$; see Figure 22. ${ }^{30}$ All relations in the hexagon for partial orders in Figure 21(b) (and its Boolean closure) can thus be written as $\bigcup \mathcal{X}$ for some $\mathcal{X} \subseteq\{=,<,>, \#\}$; for example, the relations $\leq, \geq$ and $\neq$ correspond to $=\cup<,=\cup>$ and $\langle\cup\rangle \cup \#$, respectively. As a consequence, the hexagon for partial orders in Figure 21(b) can be reformulated as in Figure 23(a). ${ }^{31}$

The Aristotelian hexagon in Figure 23(a) can be seen as a subdiagram of the Aristotelian RDH for partial orders that was shown in Figure 22. Needless to say, the latter also has many other subdiagrams, which can be exhaustively studied. For now, we will mention only two such subdiagrams: the strong JSB hexagon in Figure 23(b) and the 'contrariety square' in Figure 23(c), which are reformulations in terms of $\wp^{\cup}(\{=,<,>, \#\})$ of Aristotelian diagrams for partial orders that were originally studied by Béziau [6, Figures 3 and 9].

\footnotetext{
${ }^{30}$ Note that we can move back from partial to total orderings by imposing the totality axiom, which corresponds to assuming that $\#=\emptyset$. This assumption entails that the 16 relations of $\wp^{\cup}(\{=,<,>, \#\})$ collapse pairwise into 8 relations (this is analogous to the pairwise collapse of $\wp^{\cup}(\mathcal{O G})$ that is described toward the end of Subsection 4.5, where it is assumed that $N C D=\emptyset$ ). Ignoring the empty and universal relation, we thus find that the $16-2=14$ relations of the RDH for partial orders in Figure 22 pairwise collapse into the $\frac{16}{2}-2=6$ relations of the strong JSB hexagon for linear orders in Figure 21(a).

${ }^{31}$ We are now also in a position to explain why the hexagons in Figure 17(c) and 21(b) do not entirely consist of the same Aristotelian relations, despite the fundamental underlying analogy that was described earlier (ignoring $N C D$ vs. ignoring \#). The differences between both diagrams arise from the fact that the hexagon's top element in Figure 17(c) is $C \cup S C$, whereas in Figure 21(b) it is $\langle\cup\rangle \cup \#$. To obtain a fully perfect analogy, we would need to put $C \cup S C \cup N C D$ at the top of the hexagon in Figure 17(c), or, alternatively, $\langle\cup\rangle$ at the top of the hexagon in Figure 21(b). However, the first option is not the most plausible interpretation of Béziau's use of 'not $C D$ ' (recall Footnote 21), while the second one does not match our intuitive understanding of $\neq$ (take, for example, the partial order of set inclusion, and consider the sets $A=\{1,2\}$ and $B=\{2,3\}$ : even though neither $A \subset B$ nor $A \supset B$, we still want to be able to say that $A \neq B$ ).
} 
Figure 23. Three Aristotelian subdiagrams of the Aristotelian RDH for partial orders shown in Figure 22

(a)

$<\cup>U \#$

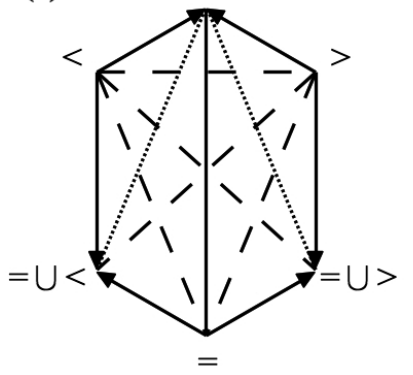

(b)

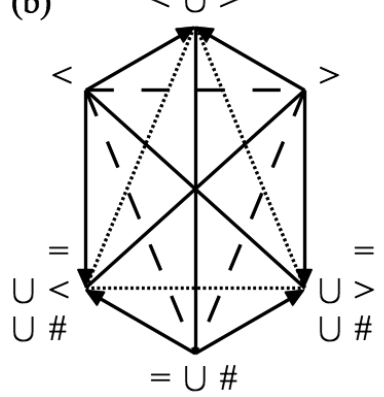

(c)

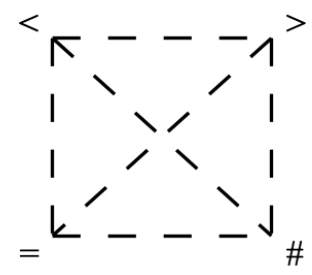

Concrete instances of the Aristotelian RDH for partial orders in Figure 22 (or any of its subdiagrams) can be obtained by 'filling in' a concrete partial order. A well-known example of a partial ordering relation is set inclusion, and thus we immediately obtain an Aristotelian RDH for set inclusion [76]. Another example, which is more relevant for our current purposes, is based on the fact that S-entailment is a partial order over the LindenbaumTarski algebra $\mathbb{B}(\mathrm{S})$ of a given logical system $\mathrm{S}$, and hence, we immediately obtain an Aristotelian RDH for entailment. This diagram is exactly the RDH for the implication geometry $\mathcal{I} \mathcal{G}$ that was described in Subsection 5.1; in particular, the abstract relations $=,<,>$ and \# are instantiated exactly as the implication relations $B I, L I, R I$ and $N I$, respectively, and hence $\wp^{\cup}(\{=,<,>, \#\})=\wp^{\cup}(\{B I, L I, R I, N I\})=\wp^{\cup}(\mathcal{I} \mathcal{G})$. This shows that the Aristotelian $\mathrm{RDH}$ for $\mathcal{I} \mathcal{G}$ is not only related to the $\mathrm{RDH}$ for $\mathcal{O G}$, as was shown in Subsection 5.1, but is also a specific instance of the RDH for partial orders.

\section{Aristotelian Diagrams for Aristotelian and Duality Relations}

In this section we conclude our study of metalogical decorations for Aristotelian diagrams. Subsection 6.1 studies some Aristotelian diagrams that are decorated with the Aristotelian relations themselves, while Subsection 6.2 studies some Aristotelian diagrams for the duality relations.

\subsection{Aristotelian Diagrams for the Aristotelian Relations}

Since the Aristotelian geometry is hybrid between the opposition and implication geometries $(\mathcal{A G} \subseteq \mathcal{O G} \cup \mathcal{I} \mathcal{G})$, many of the diagrams that were studied in Sections 4 and 5 can be viewed not only as Aristotelian diagrams for $\mathcal{O G}$ or $\mathcal{I G}$, but also as Aristotelian diagrams for $\mathcal{A G}$ itself. For example, since contrariety and subcontrariety are not only opposition relations, but also Aristotelian relations, the decoration of the Buridan octagon for strong and weak (sub)contrariety in Figure 16 can be seen as consisting of opposition relations, 
but equally well as consisting of Aristotelian relations. Still, the Aristotelian diagrams in Sections 4 and 5 do not fully do justice to the hybrid nature of $\mathcal{A G}$, since none of them has a decoration that simultaneously contains opposition and implication relations. In this subsection, we will therefore focus exactly on Aristotelian diagrams with decorations containing both opposition and implication relations.

The Aristotelian diagrams that were constructed in Sections 4 and 5 are all based on the fact that $\mathcal{O G}$ and $\mathcal{I} \mathcal{G}$ are partitions of $\mathbb{B}(\mathrm{S}) \times \mathbb{B}(\mathrm{S})$ (recall Lemma 3.5). It is well-known, however, that the Aristotelian geometry is not a partition-indeed, this was one of the main motivations for introducing $\mathcal{O G}$ and $\mathcal{I} \mathcal{G}$ in [79]-, and thus we cannot straightforwardly apply the same technique as before. In particular, it does not make sense to introduce $\wp^{\cup}(\mathcal{A G}):=\{\bigcup \mathcal{X} \mid \mathcal{X} \subseteq \mathcal{A} \mathcal{G}\}$, since the latter is not a Boolean algebra whose atoms are the Aristotelian relations.

Since the Aristotelian geometry is hybrid between the opposition and implication geometries $(\mathcal{A G} \subseteq \mathcal{O G} \cup \mathcal{I} \mathcal{G})$, it makes sense to look at the meet (i.e. the coarsest common refinement) of the partitions $\mathcal{O G}$ and $\mathcal{I} \mathcal{G}$, which is defined as follows:

$$
\mathcal{O G} \wedge \mathcal{I G}:=\{R \cap S \mid R \in \mathcal{O G}, S \in \mathcal{I} \mathcal{G}, R \cap S \neq \emptyset\} .
$$

Since the partition $\mathcal{O G} \wedge \mathcal{I} \mathcal{G}$ is a refinement of $\mathcal{O G}$ as well as $\mathcal{I} \mathcal{G}$, it follows that every Aristotelian relation can be seen as an element of the Boolean closure $\wp^{\cup}(\mathcal{O G} \wedge \mathcal{I} \mathcal{G})[31]$. Note, however, that there are $|\mathcal{O G}| \times|\mathcal{I} \mathcal{G}|=4 \times 4=16$ relations of the form $R \cap S$ for $R \in \mathcal{O G}$ and $S \in \mathcal{I} \mathcal{G}$, and only one of them is irrelevant (it can easily be shown that $C D \cap B I=\emptyset$ if the underlying logical system $S$ is consistent). In other words, we have $|\mathcal{O G} \wedge \mathcal{I} \mathcal{G}|=15$, and thus $\wp^{\cup}(\mathcal{O G} \wedge \mathcal{I} \mathcal{G})$ contains $2^{15}=32768$ relations in total, which renders it practically infeasible to systematically study Aristotelian diagrams for $\mathcal{A G}$ by viewing them as subdiagrams of some larger, Boolean closed Aristotelian diagram (in contrast to Sections 4 and 5, where all Aristotelian diagrams for $\mathcal{O G}$ and $\mathcal{I} \mathcal{G}$ were seen as subdiagrams of the Aristotelian RDH for $\mathcal{O G}$ and the Aristotelian $\mathrm{RDH}$ for $\mathcal{I} \mathcal{G}$, respectively).

We will therefore proceed in a more local fashion, and study some interesting Aristotelian diagrams for $\mathcal{A G}$ without viewing them as subdiagrams of some larger, Boolean closed Aristotelian diagram. Furthermore, many of the Aristotelian diagrams that will be studied in this subsection do not hold in full generality, i.e. for all pairs of formulas $(\varphi, \psi)$, but only if we impose certain additional conditions on the formulas, such as satisfiability or contingency. ${ }^{32}$ For each diagram that is studied in this subsection, we will therefore explicitly state the additional conditions which it depends on.

The first Aristotelian diagram that we will study contains both weak opposition and weak implication relations. Löbner $[52$, p. 55] defines the following four relations:

\footnotetext{
${ }^{32}$ Formally, this means that we intersect every relation in $\wp^{\cup}(\mathcal{O G} \wedge \mathcal{I G})$ with $\Delta_{A, B}:=$ $\{(\varphi, \psi) \mid \varphi$ satifies condition $A, \psi$ satisfies condition $B\}$, and do not work with a partition of $\mathbb{B}(\mathrm{S}) \times \mathbb{B}(\mathrm{S})$, but rather of $(\mathbb{B}(\mathrm{S}) \times \mathbb{B}(\mathrm{S})) \cap \Delta_{A, B}=\Delta_{A, B}$.
} 
FiguRE 24. Löbner's relations as decorations of (a) an Aristotelian cross (no constraints) and (b) a classical Aristotelian square (constraint: satisfiability of the first argument)

(a)

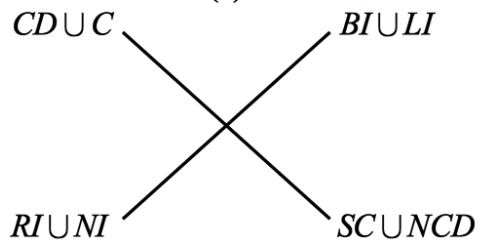

(b)

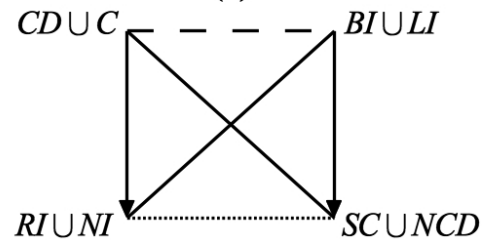

- $\varphi$ and $\psi$ are compatible iff $\mathbf{S} \not \models \neg(\varphi \wedge \psi)$,

- $\varphi$ implies $\psi$ iff $\mathrm{S}=\varphi \rightarrow \psi$,

- $\varphi$ is contrary to $\psi$ iff $S=\neg(\varphi \wedge \psi)$,

- $\varphi$ does not imply $\psi$ iff $S \not \models \varphi \rightarrow \psi$.

These four relations correspond exactly to $S C \cup N C D, B I \cup L I, C D \cup C$ and $R I \cup N I$, respectively. Löbner thus exclusively works with weak opposition and implication relations: using notation from Sections 4 and 5, his relations are $S C_{w}^{*}, L I_{w}, C_{w}$ and $R I_{w}^{*}$, respectively.

Since $\mathcal{O G}$ and $\mathcal{I} \mathcal{G}$ are partitions of $\mathbb{B}(\mathrm{S}) \times \mathbb{B}(\mathrm{S})$ (recall Lemma 3.5), it is trivial to check that $C D \cup C$ and $S C \cup N C D$ are contradictory, and also that $B I \cup L I$ and $R I \cup N I$ are contradictory. If we do not impose any additional constraints, then these two contradictions are the only Aristotelian relations that obtain between Löbner's four relations. For example, $C D \cup C$ and $B I \cup L I$ do not stand in any Aristotelian relation at all:

- $(p \wedge \neg p, p) \in C D \cup C$ and $(p \wedge \neg p, p) \in B I \cup L I$, so $(C D \cup C) \cap(B I \cup L I) \neq \emptyset$,

so $C D \cup C$ and $B I \cup L I$ are neither contradictory nor contrary,

- $(p, q) \notin C D \cup C$ and $(p, q) \notin B I \cup L I$, so $(C D \cup C) \cup(B I \cup L I) \neq \mathbb{B}(\mathrm{CPL}) \times \mathbb{B}(\mathrm{CPL})$, so $C D \cup C$ and $B I \cup L I$ are not subcontrary,

- $(p, \neg p) \notin(C D \cup C) \cap(B I \cup L I)$ and $(p, \neg p) \in C D \cup C$, so $(C D \cup C) \cap(B I \cup L I) \neq C D \cup C$, so there is no subalternation from $C D \cup C$ to $B I \cup L I$,

- $(p, p) \notin(C D \cup C) \cap(B I \cup L I)$ and $(p, p) \in B I \cup L I$, so $(C D \cup C) \cap(B I \cup L I) \neq B I \cup L I$, so there is no subalternation from $B I \cup L I$ to $C D \cup C$.

Without imposing any additional constraints, Löbner's relations thus constitute a 'degenerated' Aristotelian square (or 'cross'), which is shown in Figure 24(a).

If we make the additional assumption that the relations' first argument is satisfiable, however, the situation changes quite drastically. For example, based on this assumption, it can be shown that $C D \cup C$ and $B I \cup L I$ are contrary to each other: it is still trivially the case that $(C D \cup C) \cup(B I \cup L I)$ 
is not the universal relation over $\mathbb{B}(\mathrm{S})$, and to see that $(C D \cup C) \cap(B I \cup L I)$ is empty, note that $(\varphi, \psi) \in(C D \cup C) \cap(B I \cup L I)$ entails that $\mathrm{S} \models \neg(\varphi \wedge \psi)$ and $\mathrm{S} \models \varphi \rightarrow \psi$, and thus $\mathrm{S} \models \neg \varphi$, which contradicts our assumption that $\varphi$ is satisfiable. In exactly the same way, it can be shown that $S C \cup N C D$ and $R I \cup$ $N I$ are subcontrary to each other, and that there are subalternations from $B I \cup L I$ to $S C \cup N C D$ and from $C D \cup C$ to $R I \cup N I$. On the assumption that their first argument is satisfiable, Löbner's relations thus end up constituting a classical Aristotelian square, which is shown in Figure 24(b). Although Löbner himself did not explicitly draw this metalogical square, he recognized that his four relations "constitute another Aristotelian square too, implication $[B I \cup L I]$ implying compatibility $[S C \cup N C D]$ and so on" [52, p. 55].

In Seuren [72, p. 11] we find another Aristotelian diagram that contains both opposition and implication relations. Seuren considers the same four relations as Löbner (although he calls them differently), and adds two more, viz. 'strict compatibility' and its complement. As indicated by Seuren himself [72, p. 3], the relation of strict compatibility is better known as logical independence or unconnectedness [79, Footnote 43], ${ }^{33}$ and is defined as follows:

$\varphi$ and $\psi$ are unconnected iff $\mathrm{S} \not \models \neg(\varphi \wedge \psi)$ and $\mathrm{S} \not \models \varphi \vee \psi$ and $\mathrm{S} \not \forall \varphi \rightarrow \psi$ and $\mathrm{S} \not \models \psi \rightarrow \varphi$.

It should be clear that Seuren's notion of strict compatibility can be represented as $N C D \cap N I$. Making use of Lemma 3.5, the complement of strict compatibility can be represented as follows:

$\operatorname{not}(N C D \cap N I)=\operatorname{not}(N C D) \cup \operatorname{not}(N I)=(C D \cup C \cup S C) \cup(B I \cup L I \cup R I)$.

Strict compatibility and its complement are (by definition) contradictory to each other. Furthermore, even without making any additional assumptions about the relations' arguments, it can easily be shown that these two new relations enter into Aristotelian relations with Löbner's original four; for example, $N C D \cap N I$ is contrary to $C D \cup C$ and to $B I \cup L I$, and stands in subalternation to $S C \cup N C D$ and to $R I \cup N I$. In total, Seuren's relations thus constitute the Aristotelian hexagon shown in Figure 25(a); this type of hexagon is relatively unknown, but has recently been studied and called an 'unconnected-4' hexagon, since it contains exactly four pairs of vertices that do not stand in any Aristotelian relation at all [32, 78, 81].

If we do make the additional assumption that the relations' first argument is satisfiable, then the cross constituted by Löbner's four relations again turns into a classical Aristotelian square (recall Figure 24), and the hexagon as a whole turns into a more familiar JSB hexagon, as shown in Figure 25(b), and originally in [72, Figure 4]. It should be noted that this is a weak JSB hexagon, since $(C D \cup C) \cup(B I \cup L I) \cup(N C D \cap N I) \neq \mathbb{B}(\mathrm{S}) \times \mathbb{B}(\mathrm{S}) .{ }^{34}$

\footnotetext{
${ }^{33}$ See Footnote 2 of this paper for the relation between unconnectedness and the informativity account of the Aristotelian relations that is presented in [79].

${ }^{34}$ For example, $(p \vee \neg p, p) \in S C \cap R I$, and hence $(p \vee \neg p, p) \notin C D \cup C$ and $(p \vee \neg p, p) \notin B I \cup L I$ and $(p \vee \neg p, p) \notin N C D \cap N I$.
} 
FiguRE 25. Seuren's relations as decorations of (a) an 'unconnected-4' hexagon (no constraints) and (b) a weak JSB hexagon (constraint: satisfiability of the first argument)
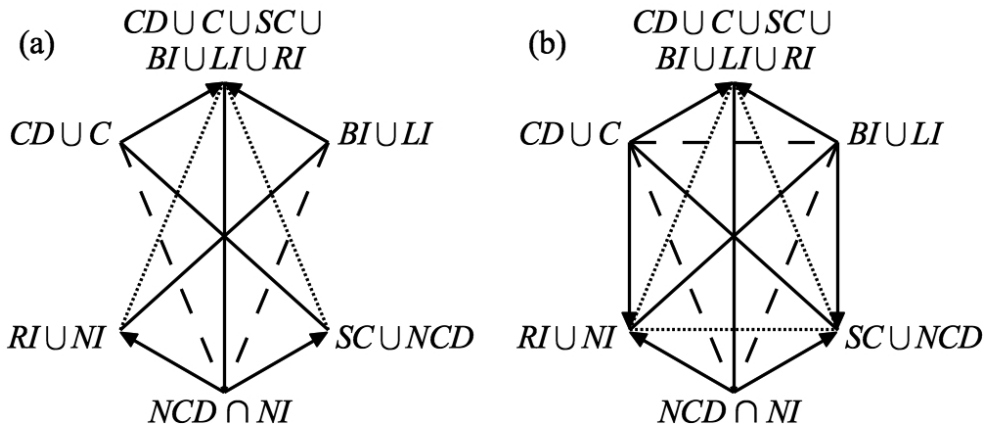

FiguRE 26. (a) Variant of Löbner's square (constraint: the first argument is not a tautology), (b) Aristotelian octagon (constraint: contingency of both arguments)

(a)

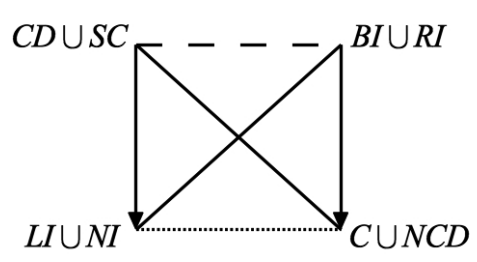

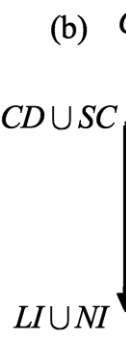

Löbner's squares in Figure 24 are decorated with weak relations $C_{w}$ and $L I_{w}$ and the 'dually' weak relations $S C_{w}^{*}$ and $R I_{w}^{*}$. A variant of Löbner's square can be obtained by changing which relations are 'ordinarily weak' and which ones are 'dually weak', i.e. by considering the relations $C_{w}^{*}=C \cup N C D$, $L I_{w}^{*}=L I \cup N I, S C_{w}=C D \cup S C$ and $R I_{w}=B I \cup R I$. If we do not make any additional assumptions, these four new relations again constitute a degenerated Aristotelian square, just like Löbner's four original ones (see Figure 24(a)). However, if we make the additional assumption that the relations' first argument is not a tautology, ${ }^{35}$ then they turn out to constitute a classical Aristotelian square, which is shown in Figure 26(a).

\footnotetext{
${ }^{35}$ Note the close connection between the conditions that are needed to turn the degenerated square into a classical square: for Löbner's original four relations, the condition is that the first argument is satisfiable (i.e. $\mathbf{S} \not \models \neg \varphi$ ), for the four new relations, the condition is that the first argument is not a tautology (i.e. $\mathrm{S} \not \models \varphi$ ).
} 
Löbner's original square in Figure 24(b) and its variant in Figure 26(a) can be combined with each other, thereby yielding an Aristotelian octagon. To ensure that both squares are classical (i.e. not degenerated), we have to assume that the relations' first argument is satisfiable and not a tautology, i.e. that it is contingent. If we additionally assume that the relations' second argument is also contingent, then the relations of the two squares enter into further Aristotelian relations with each other; for example, it can be shown that $C D \cup C$ (from Löbner's original square) and $B I \cup R I$ (from the variant to Löbner's square) are contrary to each other, ${ }^{36}$ and also that $B I \cup L I$ (from Löbner's original square) and $C D \cup S C$ (from the variant) are contrary to each other. $^{37}$ The resulting metalogical octagon is shown in Figure $26(\mathrm{~b}){ }^{38}$ This type of Aristotelian octagon has hitherto not been studied in any systematic way, but it can be found in $[23,37,45,47]$, where it receives an object-logical decoration consisting of categorical statements from syllogistics. In [31] is is shown that the octagon's object-logical (syllogistic) decoration is intimately related to the metalogical decoration that has been discussed here.

To finish this subsection, we will study two different ways to turn the weak JSB hexagon in Figure 25(b) into a strong JSB hexagon. Note that the reason for the JSB hexagon in Figure 25(b) being weak is that its contrariety triangle consists of $C D \cup C, B I \cup L I$ and $N C D \cap N I$, and thus lacks $S C$ and $R I$ (see Footnote 34 ); in order to obtain a strong JSB hexagon, we thus need to add these two relations to the hexagon's contrariety triangle. One possibility is to combine both relations into $S C \cup R I$, and add this to $N C D \cap N I$, which results in the Aristotelian hexagon in Figure 27(a). If we make the assumption that the relations' first argument is satisfiable and that their second argument is contingent, then this hexagon can be shown to be a strong JSB hexagon. An alternative possibility is to add $S C$ to $C D \cup C$, and $R I$ to $B I \cup L I$, which results in the Aristotelian hexagon in Figure 27(b). This second possibility is conceptually more elegant than the first one, since it better respects the distinction between the opposition and implication geometries. ${ }^{39}$ Furthermore, if we make the assumption that both of the relations' arguments are contingent, then the diagram in Figure 27(b) can be shown to be a strong JSB hexagon as well.

\footnotetext{
${ }^{36}$ Proof: it is trivial that $(C D \cup C) \cup(B I \cup R I)$ is not the universal relation over $\mathbb{B}(S)$; to see that $(C D \cup C) \cap(B I \cup R I)=\emptyset$, note that $(\varphi, \psi) \in(C D \cup C) \cap(B I \cup R I)$ entails that $\mathrm{S} \models \neg(\varphi \wedge \psi)$ and $\mathrm{S} \models \psi \rightarrow \varphi$, and thus $\mathrm{S} \models \neg \psi$, which contradicts our assumption that $\psi$ is (contingent and thus) satisfiable.

${ }^{37}$ Proof: it is trivial that $(B I \cup L I) \cup(C D \cup S C)$ is not the universal relation over $\mathbb{B}(S)$; to see that $(B I \cup L I) \cap(C D \cup S C)=\emptyset$, note that $(\varphi, \psi) \in(B I \cup L I) \cap(C D \cup S C)$ entails that $\mathrm{S} \models \varphi \rightarrow \psi$ and $\mathrm{S} \models \varphi \vee \psi$, and thus $\mathrm{S} \models \psi$, which contradicts our assumption that $\psi$ is (contingent and thus) not a tautology.

${ }^{38}$ Although the Aristotelian octagon in Figure 26(b) was obtained by combining the two classical Aristotelian squares in Figure 24(b) and 26(a), it can also be seen as the result of combining the degenerated squares in the centers of the two Buridan octagons in Figures 16 and 20 .

${ }^{39}$ The hexagon in Figure 27(b) is also intimately related to the information perspective on $\mathcal{O G}$ and $\mathcal{I G}$ developed in [79].
} 
FiguRE 27. (a) strong JSB hexagon (constraint: satisfiability of the first argument and contingency of the second argument), (b) another strong JSB hexagon (constraint: contingency of both arguments)
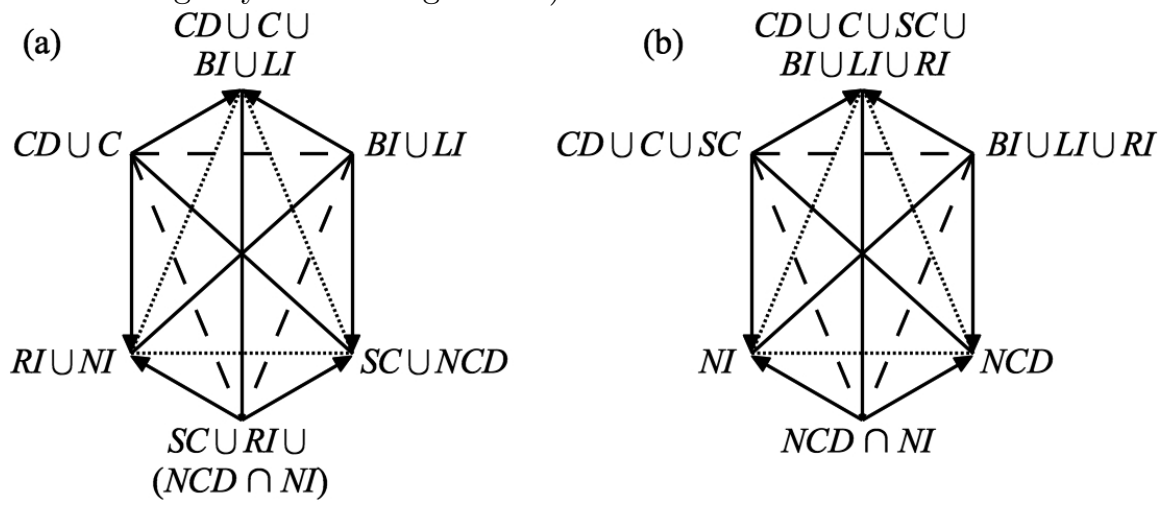

\subsection{Aristotelian Diagrams for the Duality Relations}

We now turn to Aristotelian diagrams for the duality relations ID, ENEG, INEG and DUAL, which were introduced in Subsection 3.2. Just like the Aristotelian relations (but unlike the opposition and implication relations), the duality relations do not constitute a partition of $\mathbb{B}(S) \times \mathbb{B}(S)$ : they are neither jointly exhaustive (for example, $p$ and $q$ do not stand in any duality relation at all), nor mutually exclusive (as was explained in Subsection 3.2, the existence of operators that are their own duals or internal negations entails that ID $\cap$

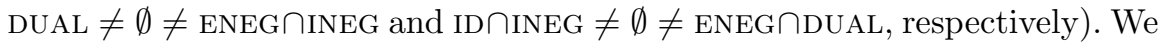
thus find ourselves in a situation that is highly similar to the one described at the beginning of the previous subsection, and we will therefore use the same strategy for dealing with it: we will proceed in a local fashion, and construct various interesting Aristotelian diagrams for the duality geometry $\mathcal{D G}$, without viewing them as subdiagrams of some larger, Boolean closed Aristotelian diagram. Furthermore, just as in the previous subsection, many of the Aristotelian diagrams that will be studied here do not hold in full generality, i.e. for all pairs of operators $\left(O_{1}, O_{2}\right)$, but only if we impose certain additional conditions on the operators, such as not being their own dual or internal negation. ${ }^{40}$ For each diagram that is studied in this subsection, we will therefore again explicitly state the additional conditions it depends on.

\footnotetext{
${ }^{40}$ Recall that in Subsection 6.1, we imposed additional conditions on the Aristotelian relations' two arguments independently — for example, it made sense to require the first formula to be satisfiable, and the second formula to be contingent; see Figure 27(a). Since the duality relations are functional, however, it can easily be shown that a duality relation's first argument satisfies a certain condition iff its second argument satisfies that same condition; for example, if two operators $O_{1}$ and $O_{2}$ stand in some duality relation $R \in \mathcal{D G}$, then we have the following chain of equivalences: $O_{2}$ is self-dual iff DUAL $\left(O_{2}\right)=O_{2}$ iff $R\left(\operatorname{Dual}\left(O_{2}\right)\right)=R\left(O_{2}\right)$ iff $\operatorname{dual}\left(R\left(O_{2}\right)\right)=R\left(O_{2}\right)$ iff $\operatorname{DuAL}\left(O_{1}\right)=O_{1}$ iff $O_{1}$ is self-dual.
} 
FiguRE 28. (a) degenerated Aristotelian square for duality relations (constraints: none), (b) classical Aristotelian square for the same duality relations (constraint: no operators that are their own dual), (c) 'unconnected-8' hexagon for the duality relations (constraints: none)

(a)

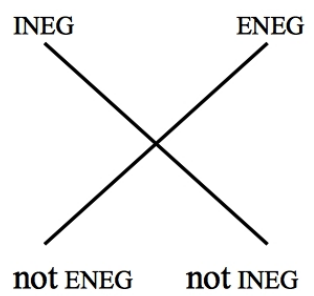

(b)

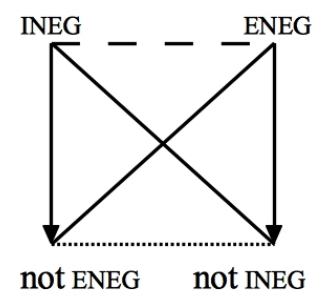

(c)

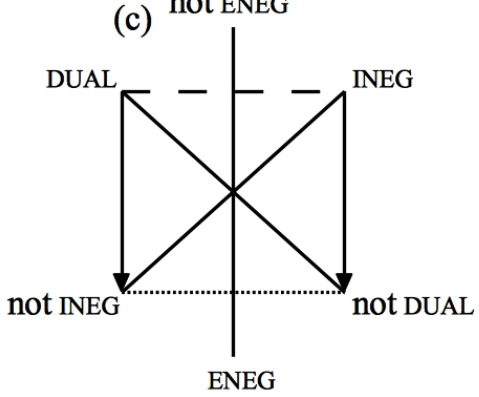

We begin by defining an Aristotelian square for the duality relations INEG and ENEG, and their complements, which will be denoted as 'not INEG' and 'not ENEG'. ${ }^{41}$ By definition, INEG and 'not INEG' are contradictory to each other, and similarly for ENEG and 'not ENEG'. Whether there are any other Aristotelian relations holding between these four relations, however, depends on the additional conditions we are willing to impose on the relations' arguments. It can be shown that without additional conditions, there are no Aristotelian relations besides the two aforementioned contradictions, and we thus obtain the 'degenerated' square shown in Figure 28(a). In particular, note that INEG and ENEG are not contrary to each other, since if the operator $O$ is self-dual, then $\operatorname{INEG}(O)=\operatorname{EnEG}(O)$, and thus INEGกENEG $\neq \emptyset$. However, if we impose the additional condition that the relations' arguments should be operators that are not self-dual, then it does hold that INEG $\cap$ ENEG $=\emptyset$, which (together with the trivial fact that INEG $\cup$ ENEG is not the universal relation over $\mathbb{B}^{\mathbb{A}^{n}}:=\left\{O \mid O: \mathbb{A}^{n} \rightarrow \mathbb{B}\right\}$ ) implies that INEG and ENEG are contrary to each other. Similarly, if we exclude self-dual operators, we also find two subalternations and a subcontrariety, and thus obtain the classical Aristotelian square shown in Figure 28(b).

A more general picture can be obtained by considering Aristotelian diagrams that simultaneously contain the non-trivial (i.e. non-ID) duality relations ENEG, INEG and DUAL. By definition, these three relations are contradictory to their complements, i.e. 'not ENEG', 'not INEG' and 'not DUAL', respectively. Furthermore, it can be shown that DUAL and INEG are contrary

Therefore, in this subsection we will always impose the same condition(s) on both of the duality relations' arguments.

${ }^{41}$ Note that because of our 'local' approach, we cannot write INEG $=\bigcup \mathcal{X}$ and 'not INEG' $=\bigcup \mathcal{Y}$ for some sets of relations $\mathcal{X}, \mathcal{Y}$. Of course, if we were working with a partition $\mathcal{P}$ such that $\mathcal{D G} \subseteq \wp^{\cup}(\mathcal{P})$, then we would have INEG $=\bigcup \mathcal{X}$ for some $\mathcal{X} \subseteq \mathcal{P}$, and hence also 'not INEG' $=\bigcup(\mathcal{P}-\mathcal{X})$. Similar remarks apply to ENEG. 
to each other, since DUAL $\cap$ INEG $=\emptyset$ : if there were operators $O_{1}, O_{2}: \mathbb{A}^{n} \rightarrow \mathbb{B}$ such that $\left(O_{1}, O_{2}\right) \in$ DUAL $\cap$ INEG, then $\operatorname{DUAL}\left(O_{1}\right)=O_{2}=\operatorname{INEG}\left(O_{1}\right)$, so $\operatorname{ENEG}\left(O_{2}\right)=\operatorname{EnEG}\left(\operatorname{InEG}\left(O_{1}\right)\right)=\operatorname{DuAL}\left(O_{1}\right)=O_{2}$, and thus $\mathbb{B}$ would be the trivial Boolean algebra in which $\perp_{\mathbb{B}}=\top_{\mathbb{B}}$ (recall Footnote 5$)$. For essentially the same reason, it also holds that 'not INEG' and 'not DUAL' are subcontrary to each other, and also that there are subalternations from DUAL to 'not INEG' and from INEG to 'not DUAL'. However, if we do not make any additional assumptions about the operators, then there are no other Aristotelian relations among DUAL, INEG, ENEG and their complements besides the ones that have just been mentioned, and we thus end up with the Aristotelian hexagon shown in Figure 28(c). This type of hexagon has recently been called an 'unconnected-8' hexagon, since it contains exactly 8 pairs of vertices that do not stand in any Aristotelian relation at all [32]. ${ }^{42}$ Such 'unconnected-8' hexagons have not been studied extensively so far, but it is known that representing them with bitstrings requires bitstring of length at least 5 (unlike all other types of Aristotelian hexagons, which can be represented with bitstrings of length 3 or 4 ).

If we now make the additional assumption that the duality relations' arguments should be operators that are not their own duals, it holds that $\mathrm{INEG} \cap \mathrm{ENEG}=\emptyset$, and we thus find a contrariety between INEG and ENEG, and also the corresponding subcontrariety and two subalternations (recall the transition from the degenerated square in Figure 28(a) to the classical square in Figure 28(b)); the hexagon as a whole thus turns into an 'unconnected-4' hexagon, which is shown in Figure 29(a). Completely analogously, if we instead make the additional assumption that the duality relations' arguments should be operators that are not their own internal negations, it holds that $\mathrm{DUAL} \cap \mathrm{ENEG}=\emptyset$, and we thus find a contrariety between DUAL and ENEG, and also the corresponding subcontrariety and two subalternations; the hexagon as a whole again turns into an 'unconnected-4' hexagon, which is shown in Figure 29(b). Note that although the Aristotelian hexagons in Figure 29(a) and (b) both belong to the 'unconnected-4' family, there is still a crucial difference between them, which has to do with the 'distribution' of unconnectedness across the hexagon (i.e. which duality relations stand/do not stand in some Aristotelian relation to each other).

Finally, if we simultaneously impose the two additional assumptions that the duality relations' arguments should be operators that are neither their own duals nor their own internal negations, then the resulting hexagon turns out to be a JSB hexagon, which is shown in Figure 29(c). It should be noted that this is a weak JSB hexagon, since DUAL $\cup$ INEG $\cup$ ENEG is not the universal relation over the class $\mathbb{B}^{\mathbb{A}^{n}}$ of all operators $O: \mathbb{A}^{n} \rightarrow \mathbb{B}$.

${ }^{42}$ Note the terminological analogy with the 'unconnected-4' hexagon. 
FiguRE 29. (a) 'unconnected-4' hexagon for the duality relations (constraint: no operators that are their own dual), (b) 'unconnected-4' hexagon for the duality relations (constraint: no operators that are their own internal negation), (c) weak JSB hexagon for the duality relations (constraint: no operators that are their own dual or internal negation)

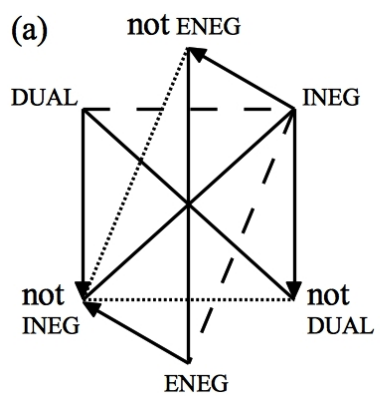

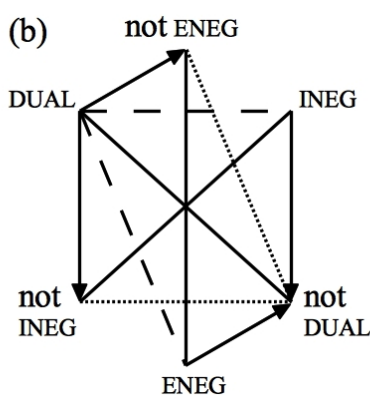

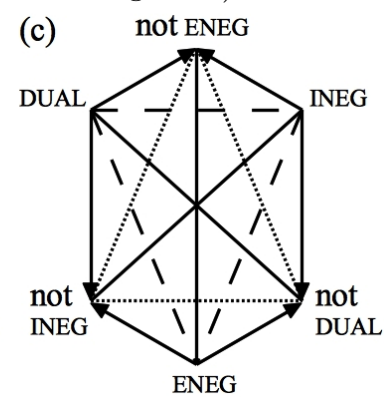

\section{Duality Diagrams for the Opposition/Implication Relations}

In Sections 4, 5 and 6, we showed that the opposition, implication, Aristotelian, and duality geometries yield various interesting metalogical decorations of Aristotelian diagrams. In this section and the next one, we will discuss how these four geometries can also be used to decorate another type of logical diagrams, viz. duality diagrams. In this section, we will focus on duality diagrams for the opposition relations (Subsection 7.1), and also make some brief comments about duality diagrams for the implication relations (Subsection 7.2).

\subsection{Duality Diagrams for the Opposition Relations}

We start by studying duality diagrams for the opposition relations. At first sight, it might look like a 'category mistake' to talk about duality relations holding between opposition relations, since in Subsection 3.2 the duality relations were defined between operators, rather than relations (recall Definition 3.6). However, every relation $R$ can naturally be associated with an operator, viz. its characteristic function $\chi_{R}$; when the characteristic functions $\chi_{R}$ and $\chi_{S}$ stand in some duality relation, then in a derived sense, the relations $R$ and $S$ themselves can also be said to stand in that duality relation. In particular, since each opposition relation $R \in \mathcal{O} \mathcal{G}_{\mathrm{S}}$ is a binary relation over the Lindenbaum-Tarski algebra $\mathbb{B}(S)$, its characteristic function $\chi_{R}$ looks as follows:

$\chi_{R}: \mathbb{B}(\mathrm{S}) \times \mathbb{B}(\mathrm{S}) \rightarrow\{0,1\}:([\varphi],[\psi]) \mapsto \chi_{R}([\varphi],[\psi]):= \begin{cases}1 & \text { if }([\varphi],[\psi]) \in R, \\ 0 & \text { if }([\varphi],[\psi]) \notin R .\end{cases}$

Since $\{0,1\}$ can be seen as a Boolean algebra (viz. the smallest non-trivial Boolean algebra), the operator $\chi_{R}$ is of the right 'type', and Definition 3.6 
is applicable to it. To illustrate this, consider item 2a of Lemma 3.3, which states the following:

$$
\text { for all formulas } \varphi, \psi: \quad C(\varphi, \psi) \quad \text { iff } \quad S C(\neg \varphi, \neg \psi) \text {. }
$$

Reformulating this in terms of characteristic functions, we get:

for all formulas $\varphi, \psi: \quad \chi_{C}(\varphi, \psi)=1 \quad$ iff $\quad \chi_{S C}(\neg \varphi, \neg \psi)=1$.

Now, in the smallest non-trivial Boolean algebra $\{0,1\}$, the biconditional $x=1 \Leftrightarrow y=1$ is equivalent to $x=y$, and thus we get:

for all formulas $\varphi, \psi: \quad \chi_{C}(\varphi, \psi)=\chi_{S C}(\neg \varphi, \neg \psi)$.

By Definition 3.6, this means exactly that $\operatorname{INEG}\left(\chi_{C}, \chi_{S C}\right)$, i.e. $\chi_{C}$ and $\chi_{S C}$ are each other's internal negation. Moving from characteristic functions to the opposition relations themselves, we can also say, in a derived sense, that INEG $(C, S C) .{ }^{43}$ Completely analogously, items 1a and 4a of Lemma 3.3 entail that INEG $(C D, C D)$ and INEG $(N C D, N C D)$, i.e. $C D$ and $N C D$ are their own internal negations.

Similar remarks also apply to non-atomic relations of $\wp^{\cup}(\mathcal{O G})$. Consider, for example, the relations $C D \cup C \cup N C D$ and $C D \cup S C \cup N C D$ : it follows immediately from items $1 \mathrm{a}, 2 \mathrm{a}$ and $4 \mathrm{a}$ of Lemma 3.3 that

for all formulas $\varphi, \psi:(\varphi, \psi) \in C D \cup C \cup N C D$ iff $\quad(\neg \varphi, \neg \psi) \in C D \cup S C \cup N C D$.

Reformulating this in terms of characteristic functions, we get

for all formulas $\varphi, \psi: \chi_{C D \cup C \cup N C D}(\varphi, \psi)=1 \quad$ iff $\quad \chi_{C D \cup S C \cup N C D}(\neg \varphi, \neg \psi)=1$ and hence

for all formulas $\varphi, \psi: \quad \chi_{C D \cup C \cup N C D}(\varphi, \psi)=\chi_{C D \cup S C \cup N C D}(\neg \varphi, \neg \psi)$, which means exactly that INEG $(C D \cup C \cup N C D, C D \cup S C \cup N C D)$.

We can also study other duality relations holding between the opposition relations. For example, since $\mathcal{O G}$ is a partition of $\mathbb{B}(\mathrm{S}) \times \mathbb{B}(\mathrm{S})$ (recall Lemma 3.5), it follows that

for all formulas $\varphi, \psi: \quad(\varphi, \psi) \in C D \quad$ iff $\quad(\varphi, \psi) \notin C \cup S C \cup N C D$.

Reformulating this in terms of characteristic functions, we get

for all formulas $\varphi, \psi: \quad \chi_{C D}(\varphi, \psi)=1 \quad$ iff $\quad \chi_{C \cup S C \cup N C D}(\varphi, \psi)=0$.

\footnotetext{
${ }^{43}$ The idea that contrariety and subcontrariety are each others internal negation is not entirely new. A closely related idea can already be found in the Summulae Logicales of the 13th century philosopher Petrus Hispanus: after he has given the definitions (which he calls 'laws) of contrariety and subcontrariety, Hispanus writes that "the law of subcontraries is the reverse of the law of contraries' [18, p. 113, our emphasis]. Interestingly, the original Latin text says that the law of subcontraries is contrary to the law of subcontraries ("lex subcontrariarum contrario modo se habet legi contrariarum' [18, p. 112, our emphasis]), which is a particularly ironic example of the confusion between Aristotelian relations and duality relations that was mentioned in Subsect. 3.2. The English translation as 'reverse is thus not literally correct, but it probably better captures what Hispanus had in mind (see [18, p. 113, Footnote 16] for the translators remarks about this issue).
} 
Figure 30. (a) Duality square for $C$ and $S C$, (b-c) degenerated duality diagrams for $C D$ and $N C D$

(a)

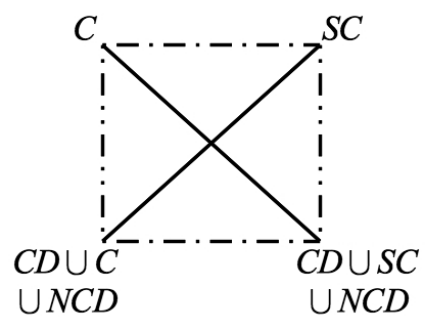

(b)

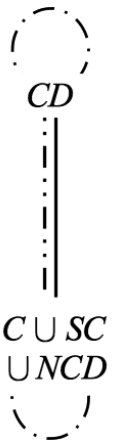

(c)

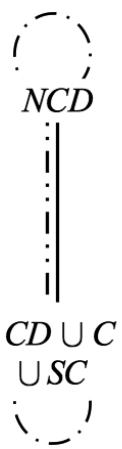

Now, in the smallest non-trivial Boolean algebra $\{0,1\}$, the biconditional $x=1 \Leftrightarrow y=0$ is equivalent to $x=\neg y$ (where $\neg$ is the complement operator of the $\{0,1\}$ Boolean algebra, i.e. $\neg y:=1-y)$, and thus we get:

for all formulas $\varphi, \psi: \quad \chi_{C D}(\varphi, \psi)=\neg \chi_{C \cup S C \cup N C D}(\varphi, \psi)$.

By Definition 3.6, this means exactly that $\operatorname{ENEG}\left(\chi_{C D}, \chi_{C \cup S C \cup N C D}\right)$, i.e. $C D$ and $C \cup S C \cup N C D$ are each other's external negation. In general, it can be shown that $\operatorname{ENEG}(\bigcup \mathcal{X}, \bigcup(\mathcal{O G}-\mathcal{X}))$ for all $\mathcal{X} \subseteq \mathcal{O G}$.

We have already established that $\operatorname{InEg}(C)=S C$ and $\operatorname{EnEg}(S C)=C D \cup$ $C \cup N C D$. Since ENEG $\circ$ INEG = DUAL, it should follow that also DUAL $(C)=$ $\operatorname{EnEG}(\operatorname{InEG}(C))=\operatorname{EnEG}(S C)=C D \cup C \cup N C D$. To verify this, note that Lemmas 3.5 and 3.3 imply that

for all formulas $\varphi, \psi: \quad(\varphi, \psi) \in C \quad$ iff $\quad(\neg \varphi, \neg \psi) \notin C D \cup C \cup N C D$, and hence

for all formulas $\varphi, \psi: \quad \chi_{C}(\varphi, \psi)=\neg \chi_{C D \cup C \cup N C D}(\neg \varphi, \neg \psi)$, which by Definition 3.6 means indeed that $\operatorname{DUAL}(C, C D \cup C \cup N C D)$.

We are now in a position to construct actual duality diagrams for the relations in $\wp^{\cup}(\mathcal{O G})$. First of all, based on the fact that $\operatorname{INEG}(C, S C)$, we can construct the duality square shown in Figure 30(a). Note that this square also visualizes facts such as $\operatorname{ENEG}(C, C D \cup S C \cup N C D)$ and $\operatorname{DUAL}(S C, C D \cup$ $S C \cup N C D)$, all of which follow from Lemmas 3.3 and 3.5. Next, since INEG $(C D, C D)$, or, functionally speaking, $\operatorname{INEG}(C D)=C D$, it follows that $\operatorname{DUAL}(C D)=\operatorname{ENEG}(\operatorname{INEG}(C D))=\operatorname{EnEG}(C D)=C \cup S C \cup N C D$, and hence we find the degenerated duality diagram in Figure 30(b), which is thus a metalogical instance of the generic degenerated duality diagram in Figure 4(c). The fact that INEG $(N C D, N C D)$ leads to a similar degenerated duality diagram, which is shown in Figure 30(c).

Next, the facts that InEg $(C D \cup C, C D \cup S C)$ and EnEg $(C D \cup S C, C \cup$ $N C D$ ) lead to the duality square in Figure 31(a), and the fact that $C D \cup N C D$ is its own internal negation - and hence $\operatorname{DUAL}(C D \cup N C D)=\operatorname{ENEG}(C D \cup$ 
Figure 31. (a) Duality square for $C D \cup C$ and other opposition relations, (b) degenerated duality diagrams for $C D \cup N C D$ and $C \cup S C$, (c) degenerated duality diagram for the empty and universal relation

(a)

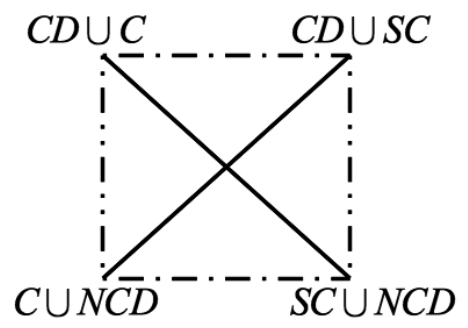

(b)

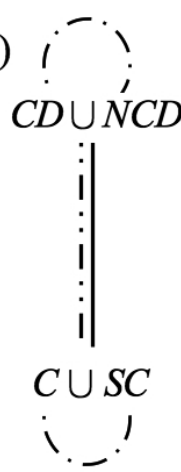

(c)

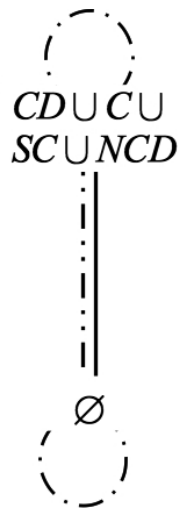

$N C D)=C \cup S C$ - leads to the degenerated duality diagram in Figure 31(b). The five duality diagrams shown in Figures $30(\mathrm{a}-\mathrm{c})$ and $31(\mathrm{a}-\mathrm{b})$ jointly exhibit all the duality relations that hold between the 14 opposition relations in the Aristotelian RDH for $\wp^{\cup}(\mathcal{O G})$ (recall Figure $\left.8(\mathrm{~b})\right) .{ }^{44}$ The only elements of $\wp^{\cup}(\mathcal{O G})$ that are not present in this RDH are the empty relation and the universal relation over $\mathbb{B}(S)$ (recall Footnote 9); these two relations turn out to be their own internal negations and each other's external negations, and thus lead to a final degenerated duality diagram, which is shown in Figure 31(c).

It is interesting to note that several (sets of) opposition relations constitute Aristotelian as well as duality diagrams. For example, we have just shown that $C, S C, C D \cup C \cup N C D$ and $C D \cup S C \cup N C D$ yield the duality square shown in Figure 30(a), but in Subsection 4.4 it was shown that these same four opposition relations also yield a classical Aristotelian square, which was shown in Figure 14(a), and is embedded as the 'vertically stretched' square inside the Buridan octagon in Figure 16(a). By contrast, $C D \cup C, C D \cup S C$, $C \cup N C D$ and $S C \cup N C D$ yield the duality square shown in Figure 31(a), but the corresponding Aristotelian diagram is not a classical square, but rather a 'degenerated' square (or 'cross'), which is embedded as the 'horizontally stretched' square inside the Buridan octagon in Figure 16(a).

These observations can be used to the argue for the conceptual independence between the Aristotelian and duality geometries. From a duality perspective, there is no difference whatsoever between the duality squares in Figures 30(a) and 31(a). The corresponding Aristotelian squares, however, are radically different from each other: the first one is a classical Aristotelian square, whereas the second one is a 'degenerated' square, i.e. an Aristotelian

\footnotetext{
${ }^{44}$ Analogously, at the object-logical level, [76] studies the duality relations that hold in the Aristotelian RDH decorated with the 14 binary propositional connectives.
} 
'cross'. As to individual relations, note, for example, that $\operatorname{DUAL}(C, C D \cup C \cup$ $N C D)$ as well as $\operatorname{DUAL}(C D \cup C, C \cup N C D)$. Switching from the duality to the Aristotelian perspective, however, we see that there is a subalternation from $C$ to $C D \cup C \cup N C D$, whereas $C D \cup C$ and $C \cup N C D$ are unconnected (i.e. they do not stand in any Aristotelian relation at all). This clearly shows that the duality relation DUAL cannot straightforwardly be identified with the Aristotelian relation of subalternation. This argument for the conceptual independence between the Aristotelian and duality geometries has already been made earlier in the literature (recall the relevant discussion in Subsection 3.2), but what we have shown here, is that the argument can be made not only based on object-logical notions (as has been done so far in the literature), but also based on metalogical notions.

We will finish this subsection with a more 'lightweight', terminological observation. Recall that in Subsection 4.4, we studied two 'weak' notions of contrariety, viz. $C_{w}=C D \cup C$ and $C_{w}^{*}=C \cup N C D$, and informally said that these two notions are 'dual' to each other, since they can both be obtained from the strong notion of contrariety $\left(C_{s}=C\right)$ by dropping the latter's $\not=$ or $\models$-condition, respectively. However, in this subsection we have shown that $\operatorname{DUAL}(C D \cup C, C \cup N C D)$, which means exactly that $\operatorname{DUAL}\left(C_{w}, C_{w}^{*}\right)$, and hence, $C_{w}$ and $C_{w}^{*}$ turn out to be also 'dual' to each other in the technically precise sense of the word! ${ }^{45}$

\subsection{Duality Diagrams for the Implication Relations}

By now, it should no longer come as a surprise that all results from the previous subsection about duality diagrams for the opposition relations can straightforwardly be transposed to duality diagrams for the implication relations (also recall the relation between Sections 4 and 5 on Aristotelian diagrams for $\mathcal{O G}$ and $\mathcal{I} \mathcal{G}$, respectively). In particular, it follows from Lemma 3.3 that $B I$ and $N I$ are their own internal negation, and that $L I$ and $R I$ are each other's internal negation. Constructing duality diagrams for the implication relations is thus entirely straightforward; however, to avoid being overly repetitive, we will not do this in full detail at this point.

\section{Duality Diagrams for Aristotelian and Duality Relations}

In this section, we finish our exploration of metalogical decorations of duality diagrams. Subsection 8.1 studies duality diagrams for the Aristotelian relations, while Subsection 8.2 studies duality diagrams for the duality relations themselves.

\subsection{Duality Diagrams for Aristotelian Relations}

Since the Aristotelian geometry is hybrid between the opposition and implication geometries $(\mathcal{A G} \subseteq \mathcal{O G} \cup \mathcal{I G})$, many of the diagrams that were studied in Section 7 can be viewed not only as duality diagrams for $\mathcal{O G}$ or $\mathcal{I} \mathcal{G}$, but

${ }^{45}$ Similar remarks can be made, of course, about $S C_{w}=C D \cup S C$ and $S C_{w}^{*}=S C \cup N C D$. 
also as duality diagrams for $\mathcal{A G}$ itself (a similar observation was made at the beginning of Subsection 6.1 about $\mathcal{O G} / \mathcal{I} \mathcal{G}$ - and $\mathcal{A G}$-based decorations of Aristotelian diagrams). However, these diagrams do not entirely exemplify the hybrid nature of $\mathcal{A G}$, since none of them has a decoration that contains opposition and implication relations simultaneously. This is not a coincidence, since the INEG-relations in these diagrams are all based on Lemma 3.3, which describes the effects of negating both arguments of an opposition or implication relation. This lemma does not establish any connection between $\mathcal{O G}$ and $\mathcal{I} \mathcal{G}$ : if both arguments of an opposition (resp. implication) relation are negated, the result is again an opposition (resp. implication) relation. This shows that there do not exist any duality diagrams (in the classical sense of the word 'duality', as captured by Definition 3.6) whose decoration contains opposition and implication relations simultaneously.

From this perspective, Lemma 3.3 stands in sharp contrast to Lemma 3.4. The latter describes the effects of negating only a single (either the first or the second) argument of an opposition or implication relation, and thereby establishes various links between $\mathcal{O G}$ and $\mathcal{I} \mathcal{G}$ : if a single argument of an opposition relation is negated, the result is an implication relation, and vice versa. In terms of duality, this means that by moving from classical duality (Definition 3.6) to generalized Post duality (Definition 3.7), we will be able to construct duality diagrams whose decoration contains opposition as well as implication relations.

To illustrate this, consider item $2 \mathrm{~b}$ of Lemma 3.4, which states the following:

$$
\text { for all formulas } \varphi, \psi: \quad C(\varphi, \psi) \text { iff } \quad L I(\varphi, \neg \psi) \text {. }
$$

Reformulating this in terms of characteristic functions, we get:

$$
\text { for all formulas } \varphi, \psi: \quad \chi_{C}(\varphi, \psi)=1 \quad \text { iff } \quad \chi_{L I}(\varphi, \neg \psi)=1
$$

and hence:

$$
\text { for all formulas } \varphi, \psi: \quad \chi_{C}(\varphi, \psi)=\chi_{L I}(\varphi, \neg \psi) \text {. }
$$

By Definition 3.7, this means exactly that $\operatorname{INEG}_{2}\left(\chi_{C}, \chi_{L I}\right)$, and in a derived sense, $\operatorname{INEG}_{2}(C, L I)$. In exactly the same way, it can be shown that $\mathrm{INEG}_{2}(C D, B I), \operatorname{INEG}_{2}(S C, R I)$, and so on. Moving to non-atomic relations of $\wp^{\cup}(\mathcal{O G})$ and $\wp^{\cup}(\mathcal{I} \mathcal{G})$, we find, for example:

for all formulas $\varphi, \psi: \quad(\varphi, \psi) \in C D \cup C \quad$ iff $\quad(\varphi, \neg \psi) \in B I \cup L I$.

and hence

$$
\text { for all formulas } \varphi, \psi: \quad \chi_{C D \cup C}(\varphi, \psi)=\chi_{B I \cup L I}(\varphi, \neg \psi),
$$

which means exactly that $\operatorname{INEG}_{2}(C D \cup C, B I \cup L I)$. Analogously, it also holds that $\operatorname{INEG}_{2}(S C \cup N C D, R I \cup N I)$.

We have already established that $\operatorname{INEG}_{2}(C D \cup C)=B I \cup L I$ and (in Subsection 7.2) that $\operatorname{EnEg}(B I \cup L I)=R I \cup N I$. Since ENEG $\circ \mathrm{INEG}_{2}=$

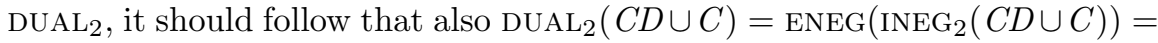


Figure 32. Three generalized Post duality squares

(a)

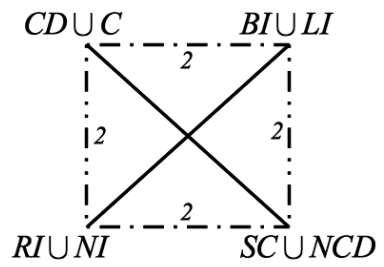

(b)

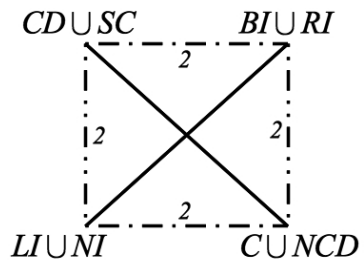

(c)

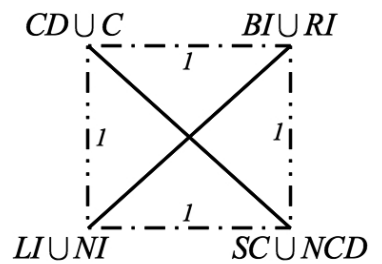

$\operatorname{ENEG}(B I \cup L I)=R I \cup N I$. To verify this, note that Lemmas 3.5 and 3.4 imply that

for all formulas $\varphi, \psi: \quad(\varphi, \psi) \in C D \cup C \quad$ iff $\quad(\varphi, \neg \psi) \notin R I \cup N I$, and hence

for all formulas $\varphi, \psi: \quad \chi_{C D \cup C}(\varphi, \psi)=\neg \chi_{R I \cup N I}(\varphi, \neg \psi)$, which by Definition 3.7 indeed means that $\operatorname{DUAL}_{2}(C D \cup C, R I \cup N I)$.

In sum, then, we find that the opposition relations $C D \cup C$ and $S C \cup$ $N C D$ together with the implication relations $B I \cup L I$ and $R I \cup N I$ can be used to decorate a generalized Post duality square, which is shown in Figure $32(\mathrm{a})$. Note that these are exactly the four relations defined by Löbner [52, p. 55], who explicitly recognized that they "themselves form a duality square with respect to the predicate [i.e. using $\mathrm{INEG}_{2}$ and $\mathrm{DUAL}_{2}$ instead of INEG and DUAL]" (emphasis added). In Subsection 6.1 we showed that these four relations also yield an Aristotelian square (recall Figure 24), and pointed out that this, too, was already recognized by Löbner. The exact nature of this Aristotelian square turned out to depend on whether or not the relations' first argument is assumed to be satisfiable - compare Figure 24(a) and (b). By contrast, the (generalized Post) duality square in Figure 32(a) does not depend on any additional assumptions.

In an analogous fashion, it can be shown that $C D \cup S C, C \cup N C D, B I \cup R I$ and $L I \cup N I$ yield another generalized Post duality square, which is shown in Figure 32(b). Note that these are exactly the four relations that were used in Subsection 6.1 to define a 'variant' to Löbner's square (recall Figure 26(a)). Furthermore, the exact nature of this Aristotelian square turned out to depend on whether or not the relations' first argument is not a tautology; by contrast, the corresponding generalized Post duality square in Figure 32(b) does not depend on any additional assumptions.

Similar results can be obtained if we work with $\mathrm{INEG}_{1}$ instead of $\mathrm{INEG}_{2}$. For example, it follows from items $1 \mathrm{a}$ and $2 \mathrm{a}$ of Lemma 3.4 that $\operatorname{INEG}_{1}(C D \cup$ $C, B I \cup R I)$, and from items $3 \mathrm{a}$ and $4 \mathrm{a}$ of the same lemma that $\operatorname{INEG}_{1}(S C \cup$ $N C D, L I \cup N I)$. Furthermore, also making use of Lemma 3.5, it is easy to check that $\mathrm{DUAL}_{1}(C D \cup C, L I \cup N I)$ and $\operatorname{DUAL}_{1}(S C \cup N C D, B I \cup R I)$. The resulting generalized Post duality square is shown in Figure 32(c). 
Figure 33. (a) Shield and spear generalized Post duality diagram corresponding to Seuren's Aristotelian hexagon in Figure 25, (b) generalized Post duality cube corresponding to the Aristotelian octagon in Figure 26(b)

(a) $\cdot-i-i$<smiles></smiles>

(b)

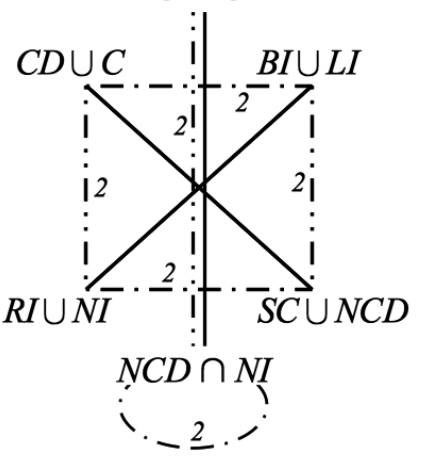

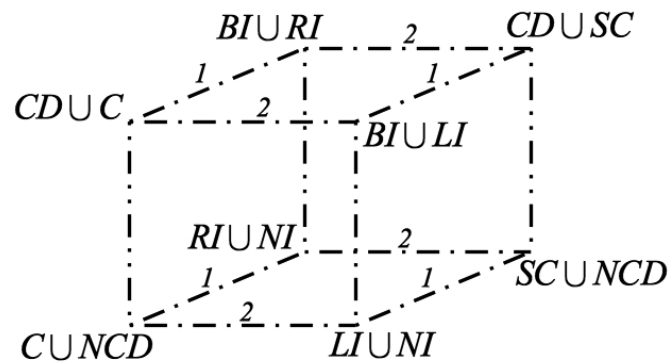

Recall that in Subsection 6.1, we also studied Seuren's Aristotelian metahexagon, which can be obtained by adding $C D \cup C \cup S C \cup B I \cup L I \cup R I$ and $N C D \cap N I$ to Löbner's four relations (recall Figure 25). Now, it follows from Lemma 3.4 that

for all formulas $\varphi, \psi: \quad(\varphi, \psi) \in N C D \cap N I \quad$ iff $\quad(\varphi, \neg \psi) \in N C D \cap N I$, and hence $\mathrm{INEG}_{2}(N C D \cap N I, N C D \cap N I)$. Analogously, it holds that $\mathrm{INEG}_{2}(C D \cup$ $C \cup S C \cup B I \cup L I \cup R I, C D \cup C \cup S C \cup B I \cup L I \cup R I)$ and $\operatorname{DUAL}_{2}(N C D \cap$ $N I, C D \cup C \cup S C \cup B I \cup L I \cup R I)$. Seuren's Aristotelian hexagon in Figure 25 thus turns out to correspond to the generalized Post duality diagram shown in Figure 33(a). This diagram consists of two 'independent' parts, viz. the duality square which was already shown in Figure 32(a), and a degenerated duality diagram containing $N C D \cap N I$ and $C D \cup C \cup S C \cup B I \cup L I \cup R I{ }^{46}{ }^{46}$ Finally, recall that in Subsection 6.1 it was shown that the exact nature of Seuren's Aristotelian hexagon depends on whether or not the relations' first argument is assumed to be satisfiable - compare Figure 25(a) and (b). By contrast, the (generalized Post) duality diagram in Figure 33(a) does not depend on any additional assumptions.

We showed in Subsection 3.2 that generalized Post duality generally gives rise to cube diagrams. Starting with $C D \cup C$ and applying the $\mathrm{INEG}_{1^{-}}$, $\mathrm{INEG}_{2}{ }^{-}, \mathrm{DUAL}_{1}{ }^{-}$and $\mathrm{DUAL}_{2}$-operators (and their combinations) to it, we find exactly the 8 relations that were shown in Subsection 6.1 to yield the Aristotelian octagon in Figure 26(b). In other words, from a duality perspective,

\footnotetext{
${ }^{46}$ The duality diagram corresponding to a JSB hexagon was called a "shield and spear" diagram by Smessaert [77, p. 180].
} 
the Aristotelian octagon in Figure 26(b) turns out to correspond to a generalized Post duality cube, which is shown in Figure 33(b). This cube has been studied before in [51, Figure 3], and it contains the (classical) duality square in Figure 31(a) and the three (generalized Post) duality squares in Figure 32 as subdiagrams on its diagonal planes. Finally, recall that in Subsection 6.1 it was shown that the exact nature of the Aristotelian octagon in Figure 26(b) depends on whether or not the relations' first and second argument are assumed to be contingent. By contrast, the corresponding generalized Post duality cube in Figure 33(b) does not depend on any additional assumptions.

\subsection{Duality Diagrams for Duality Relations}

In this subsection we will show that the duality relations can themselves be used to decorate duality diagrams. The key idea is that the duality relations are all essentially their own internal negations. Consider, for example, the ENEG-relation: for any two operators $O_{1}, O_{2}: \mathbb{A}^{n} \rightarrow \mathbb{B}$, the Boolean nature of $\neg \mathbb{B}$ and Definition 3.6 imply that ${ }^{47}$

$$
\begin{array}{lll}
\operatorname{ENEG}\left(O_{1}, O_{2}\right) & \text { iff } & \text { for all } \bar{a} \in \mathbb{A}^{n}: O_{1}(\bar{a})=\neg_{\mathbb{B}} O_{2}(\bar{a}) \\
& \text { iff } & \text { for all } \bar{a} \in \mathbb{A}^{n}: \neg_{\mathbb{B}} O_{1}(\bar{a})=\neg_{\mathbb{B}} \neg_{\mathbb{B}} O_{2}(\bar{a}) \\
& \text { iff } & \operatorname{ENEG}\left(\neg_{\mathbb{B}} O_{1}, \neg_{\mathbb{B}} O_{2}\right) .
\end{array}
$$

To make this fully precise, we will write $\mathbb{F}$ for the class of all operators $O: \mathbb{A}^{n} \rightarrow \mathbb{B}$. It is well-known that $\mathbb{F}$ is itself a Boolean algebra, whose Boolean operators are defined pointwise. For example, given operators $O_{1}, O_{2} \in \mathbb{F}$, their meet $O_{1} \wedge_{\mathbb{F}} O_{2}$ is defined to be the operator $O_{1} \wedge_{\mathbb{F}} O_{2}: \mathbb{A}^{n} \rightarrow \mathbb{B}: \bar{a} \mapsto$ $\left(O_{1} \wedge_{\mathbb{F}} O_{2}\right)(\bar{a}):=O_{1}(\bar{a}) \wedge_{\mathbb{B}} O_{2}(\bar{a})$, and similarly, the complement $\neg_{\mathbb{F}} O_{1}$ is defined to be the operator $\neg_{\mathbb{F}} O_{1}: \mathbb{A}^{n} \rightarrow \mathbb{B}: \bar{a} \mapsto\left(\neg_{\mathbb{F}} O_{1}\right)(\bar{a}):=\neg_{\mathbb{B}}\left(O_{1}(\bar{a})\right)$. Relations such as ENEG are binary relations on $\mathbb{F}$ and hence, their characteristic function is itself an operator $\chi_{\mathrm{ENEG}}: \mathbb{F}^{2} \rightarrow\{0,1\}$. Since $\mathbb{F}$ as well as $\{0,1\}$ are Boolean algebras, the operator $\chi_{\mathrm{ENEG}}$ is of the right 'type', and Definition 3.6 is applicable to it. In particular, recall that we have just showed above that

$$
\text { for all } O_{1}, O_{2} \in \mathbb{F}: \operatorname{ENEG}\left(O_{1}, O_{2}\right) \quad \text { iff } \quad \operatorname{ENEG}\left(\neg \mathbb{B} O_{1}, \neg_{\mathbb{B}} O_{2}\right) .
$$

Putting it in terms of characteristic functions, this becomes:

$$
\text { for all } O_{1}, O_{2} \in \mathbb{F}: \chi_{\mathrm{ENEG}}\left(O_{1}, O_{2}\right)=1 \quad \text { iff } \quad \chi_{\mathrm{ENEG}}\left(\neg_{\mathbb{B}} O_{1}, \neg_{\mathbb{B}} O_{2}\right)=1 \text {. }
$$

Now, in the Boolean algebra $\{0,1\}$, the biconditional $x=1 \Leftrightarrow y=1$ is equivalent to $x=y$, and thus we get:

$$
\text { for all } O_{1}, O_{2} \in \mathbb{F}: \chi_{\mathrm{ENEG}}\left(O_{1}, O_{2}\right)=\chi_{\mathrm{ENEG}}\left(\neg_{\mathbb{B}} O_{1}, \neg_{\mathbb{B}} O_{2}\right) \text {. }
$$

Because of the pointwise definition of $\neg \mathbb{F}$ in terms of $\neg \mathbb{B}$, this can be reformulated one final time, as follows:

$$
\text { for all } O_{1}, O_{2} \in \mathbb{F}: \chi_{\mathrm{ENEG}}\left(O_{1}, O_{2}\right)=\chi_{\mathrm{ENEG}}\left(\neg_{\mathbb{F}} O_{1}, \neg_{\mathbb{F}} O_{2}\right) \text {. }
$$

\footnotetext{
${ }^{47}$ We write $\bar{a}$ for the $n$-tuple $\left(a_{1}, \ldots, a_{n}\right) \in \mathbb{A}^{n}$, and also $\overline{\neg_{\mathbb{A}} a}$ for $\left(\neg_{\mathbb{A}} a_{1}, \ldots, \neg_{\mathbb{A}} a_{n}\right) \in \mathbb{A}^{n}$.
} 
By Definition 3.6, this means exactly that INEG $\left(\chi_{\mathrm{ENEG}}, \chi_{\mathrm{ENEG}}\right)$. Moving from the characteristic function $\chi_{\mathrm{ENEG}}$ to the duality relation ENEG itself, we can also say, in a derived sense, that INEG(ENEG, ENEG), i.e. ENEG is its own internal negation.

In exactly the same way, it can also be shown that the other duality relations, ID, INEG and DUAL, are their own internal negations, based on the following chains of equivalences:

$$
\begin{aligned}
& \operatorname{ID}\left(O_{1}, O_{2}\right) \quad \text { iff } \quad \text { for all } \bar{a} \in \mathbb{A}^{n}: O_{1}(\bar{a})=O_{2}(\bar{a}) \\
& \text { iff for all } \bar{a} \in \mathbb{A}^{n}: \neg_{\mathbb{B}} O_{1}(\bar{a})=\neg_{\mathbb{B}} O_{2}(\bar{a}) \\
& \text { iff } \operatorname{ID}\left(\neg_{\mathbb{B}} O_{1}, \neg_{\mathbb{B}} O_{2}\right) \text {, } \\
& \operatorname{INEG}\left(O_{1}, O_{2}\right) \quad \text { iff } \quad \text { for all } \bar{a} \in \mathbb{A}^{n}: O_{1}(\bar{a})=O_{2}\left(\overline{\bar{A}_{\mathbb{A}}}\right) \\
& \text { iff for all } \bar{a} \in \mathbb{A}^{n}: \neg_{\mathbb{B}} O_{1}(\bar{a})=\neg_{\mathbb{B}} O_{2}\left(\overline{\neg_{\mathbb{A}}}\right) \\
& \text { iff } \operatorname{INEG}\left(\neg_{\mathbb{B}} O_{1}, \neg_{\mathbb{B}} O_{2}\right) \text {, } \\
& \operatorname{DUAL}\left(O_{1}, O_{2}\right) \quad \text { iff } \quad \text { for all } \bar{a} \in \mathbb{A}^{n}: O_{1}(\bar{a})=\neg_{\mathbb{B}} O_{2}\left(\overline{\neg_{\mathbb{A}} a}\right) \\
& \text { iff for all } \bar{a} \in \mathbb{A}^{n}: \neg_{\mathbb{B}} O_{1}(\bar{a})=\neg_{\mathbb{B}} \neg_{\mathbb{B}} O_{2}\left(\overline{\neg_{\mathbb{A}}}\right) \\
& \text { iff } \operatorname{DUAL}\left(\neg_{\mathbb{B}} O_{1}, \neg_{\mathbb{B}} O_{2}\right) \text {. }
\end{aligned}
$$

We will also consider the complement of the ENEG-relation, i.e. 'not ENEG' $:=\mathbb{F}^{2}-$ ENEG. By definition of 'not ENEG', it holds that

for all $O_{1}, O_{2} \in \mathbb{F}:\left(O_{1}, O_{2}\right) \in$ 'not ENEG' iff $\quad\left(O_{1}, O_{2}\right) \notin$ ENEG.

Putting this in terms of characteristic functions, we get:

$$
\text { for all } O_{1}, O_{2} \in \mathbb{F}: \chi_{\text {'not ENEG }}{ }^{\prime}\left(O_{1}, O_{2}\right)=1 \quad \text { iff } \quad \chi_{\mathrm{ENEG}}\left(O_{1}, O_{2}\right)=0 .
$$

Now, in the Boolean algebra $\{0,1\}$, the biconditional $x=1 \Leftrightarrow y=0$ is equivalent to $x=\neg y$ (where $\neg$ is the complement operator of the $\{0,1\}$ Boolean algebra, i.e. $\neg y:=1-y)$, and thus we get:

$$
\text { for all } O_{1}, O_{2} \in \mathbb{F}: \chi_{\text {'not ENEG' }}\left(O_{1}, O_{2}\right)=\neg \chi_{\mathrm{ENEG}}\left(O_{1}, O_{2}\right) \text {. }
$$

By Definition 3.6, this means exactly that ENEG $\left(\chi_{\text {'not ENEG' }}{ }^{\prime} \chi_{\text {ENEG }}\right)$, or in a derived sense: ENEG('not ENEG', ENEG).

We have already established above that INEG(ENEG) = ENEG and just now also that ENEG(ENEG) = 'not ENEG'; it now follows that DUAL(ENEG) = $(\mathrm{ENEG} \circ \mathrm{INEG})(\mathrm{ENEG})=\operatorname{ENEG}(\operatorname{INEG}(\mathrm{ENEG}))=\operatorname{ENEG}(\mathrm{ENEG})=$ 'not ENEG', and hence DUAL(ENEG, 'not ENEG').

In exactly the same way, we can also define the relations 'not ID':= $\mathbb{F}^{2}-$ ID, 'not INEG' $:=\mathbb{F}^{2}-$ INEG and 'not DUAL' $:=\mathbb{F}^{2}-$ DUAL, and show the following:

$$
\begin{array}{ll}
\text { ENEG(ID, 'not ID'), } & \text { DUAL(ID, 'not ID'), } \\
\text { ENEG(INEG, 'not INEG'), } & \text { DUAL(INEG, 'not INEG'), } \\
\text { ENEG(DUAL, 'not DUAL'), } & \text { DUAL(DUAL, 'not DUAL'). }
\end{array}
$$

Each of the four duality relations $R \in \mathcal{D G}$ thus gives rise to a degenerated duality diagram, which is shown in Figure 34. Unfortunately, these degenerated duality diagrams are not particularly interesting. A much richer 
FIGURE 34. Degenerated duality diagrams for the duality relations

(a)<smiles>CCCC[Tl]</smiles>

$\mathrm{ID}$

not ID

i i<smiles>CCCC[Tl]</smiles>

ENEG

(b)

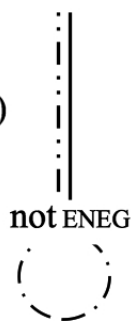<smiles>CCCC[I-]</smiles>

INEG

(c)

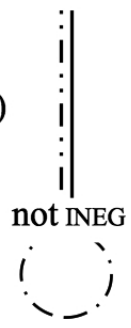<smiles>C[In]1CC[I-]C1</smiles>

DUAL

(d)

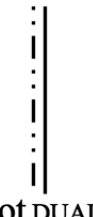

perspective can be obtained by considering the generalized Post duality relations holding between the duality relations. ${ }^{48}$ For example, note that for all operators $O_{1}, O_{2}: \mathbb{A}^{n} \rightarrow \mathbb{B}$, we have:

$$
\begin{array}{rll}
\operatorname{ID}\left(O_{1}, O_{2}\right) & \text { iff } & \text { for all } \bar{a} \in \mathbb{A}^{n}: O_{1}(\bar{a})=O_{2}(\bar{a}) \\
& \text { iff } & \text { for all } \bar{a} \in \mathbb{A}^{n}: \neg_{\mathbb{B}} O_{1}(\bar{a})=\neg_{\mathbb{B}} O_{2}(\bar{a}) \\
& \text { iff } & \operatorname{ENEG}\left(\neg_{\mathbb{B}} O_{1}, O_{2}\right) \\
& \text { iff } & \text { for all } \left.\bar{a} \in \mathbb{A}^{n}: O_{1}(\bar{a})=\neg_{\mathbb{B}}\right\urcorner_{\mathbb{B}} O_{2}(\bar{a}) \\
& \text { iff } & \operatorname{ENEG}\left(O_{1}, \neg_{\mathbb{B}} O_{2}\right) .
\end{array}
$$

These chains of equivalences can be used to show that INEG $_{1}$ (ID, ENEG) and $\mathrm{INEG}_{2}$ (ID, ENEG). Furthermore, we can also show that INEG 1 (INEG, DUAL) and $\mathrm{INEG}_{2}$ (INEG, DUAL), based on the following chains of equivalences:

$$
\begin{aligned}
& \operatorname{INEG}\left(O_{1}, O_{2}\right) \quad \text { iff } \quad \text { for all } \bar{a} \in \mathbb{A}^{n}: O_{1}(\bar{a})=O_{2}\left(\overline{\neg_{\mathbb{A}}}\right) \\
& \text { iff for all } \bar{a} \in \mathbb{A}^{n}: \neg_{\mathbb{B}} O_{1}(\bar{a})=\neg_{\mathbb{B}} O_{2}\left(\overline{\neg_{\mathbb{A}}}\right) \\
& \text { iff } \operatorname{DUAL}\left(\neg_{\mathbb{B}} O_{1}, O_{2}\right) \\
& \text { iff for all } \bar{a} \in \mathbb{A}^{n}: O_{1}(\bar{a})=\neg \mathbb{B} \neg \mathbb{B} O_{2}\left(\overline{\urcorner_{\mathbb{A}}}\right) \\
& \text { iff } \operatorname{DUAL}\left(O_{1}, \neg_{\mathbb{B}} O_{2}\right) \text {. }
\end{aligned}
$$

We have just shown that $\mathrm{INEG}_{i}(\mathrm{ID})=$ ENEG and $\mathrm{INEG}_{i}(\mathrm{INEG})=$ DUAL (for $i=1,2$ ). Using this, we find

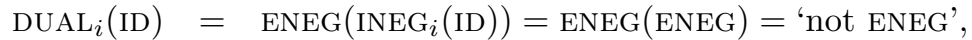

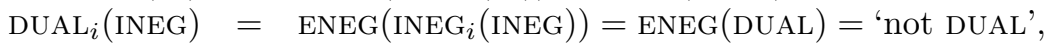

and hence $\operatorname{DUAL}_{i}\left(\mathrm{ID}\right.$, 'not ENEG') and $\operatorname{DUAL}_{i}(\mathrm{INEG}$, 'not DUAL') (for $i=1,2)$.

\footnotetext{
${ }^{48}$ In this paper we will focus on generalized Post duality relations holding between the 'classical' duality relations; in other words, we will define 'classical' duality decorations for generalized Post duality diagrams. Of course, one could also study (classical and/or generalized Post) duality relations holding between the generalized Post duality relations themselves, or, in other words, define generalized Post duality decorations for (classical and/or generalized Post) duality diagrams. This, however, will be left for another paper.
} 
Figure 35. Generalized Post duality square for ID and ENEG

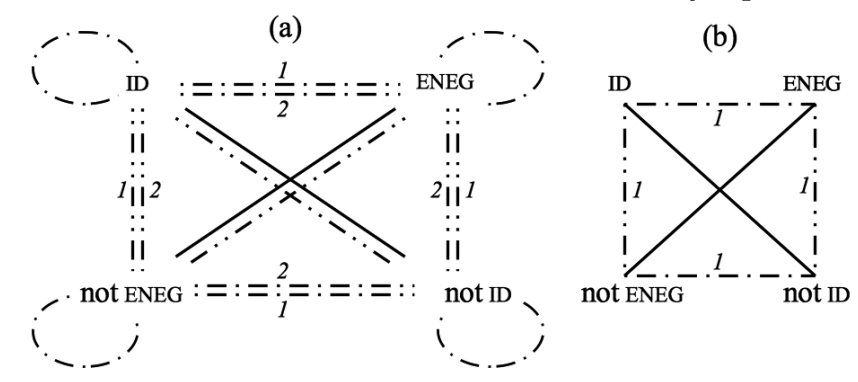

(c)

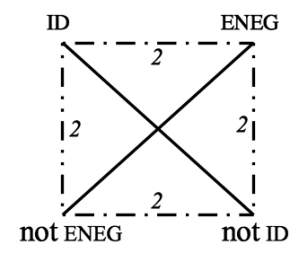

Figure 36. Generalized Post duality square for INEG and DUAL

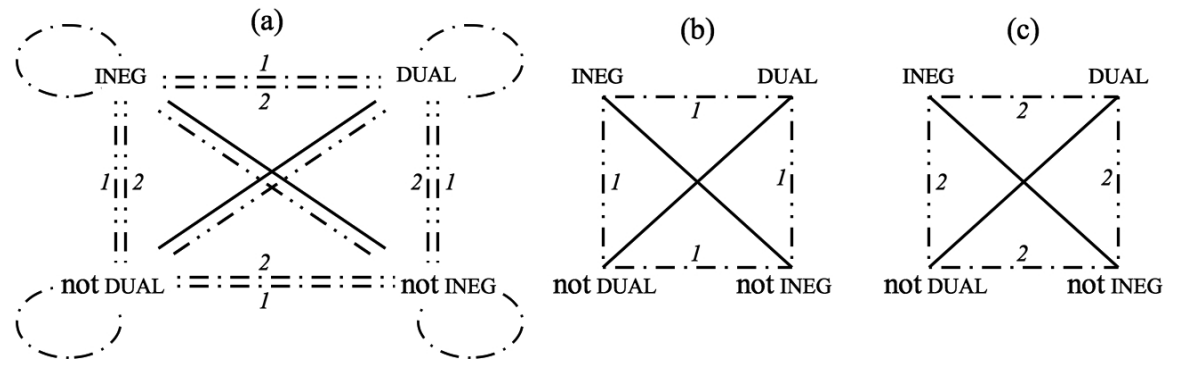

By going from 'classical' to generalized Post duality, the degenerated duality diagrams for ID and ENEG in Figure 34(a) and (b) thus 'click together' to yield a generalized Post duality square, which is shown in Figure $35(\mathrm{a})$. Since $\mathrm{INEG}_{1}$ (ID) $=\mathrm{ENEG}=\mathrm{INEG}_{2}$ (ID), this square can actually be seen as a degenerated generalized Post duality cube (see Figure 6 in Subsection 3.2). Furthermore, if we ignore the classical INEG- and DUAL-relations, then this square can be decomposed into a square for $\mathrm{INEG}_{1} / \mathrm{DUAL}_{1}$ and one for $\mathrm{INEG}_{2} / \mathrm{DUAL}_{2}$, which are shown in Figure 35(b) and (c), respectively.

Finally, the degenerated duality diagrams for INEG and DUAL in Figure 34(c) and (d) also 'click together' to yield another generalized Post duality square, which is shown in Figure 36(a). We can make the same remarks about this square as about the one in Figure 35(a); in particular, if we ignore the classical INEG- and DUAL-relations, it again decomposes into a square for $\mathrm{INEG}_{1} / \mathrm{DUAL}_{1}$ and one for $\mathrm{INEG}_{2} / \mathrm{DUAL}_{2}$, which are shown in Figure 36(b) and (c), respectively.

\section{Conclusion}

When dealing with logical diagrams, we can distinguish between a diagram's type and its decoration. The diagram's decoration consists of the formulas/notions that it represents, while its type is determined by the kind of 
logical relations holding between those formulas/notions. ${ }^{49}$ Consider, for example, the square diagram in Figure 2(b): this is an Aristotelian square for (formulas from) the modal logic S5, so we can say that its type is Aristotelian, while its decoration is based on S5.

In this paper, we have studied metalogical diagrams, whose types and decorations consist of logical relations. In particular, we have studied two diagram types, viz. Aristotelian diagrams and duality diagrams, and four kinds of diagram decorations, viz. based on relations from the opposition geometry $(\mathcal{O G})$, the implication geometry $(\mathcal{I} \mathcal{G})$, the Aristotelian geometry $(\mathcal{A G})$ and the duality geometry $(\mathcal{D G})$. A high-level overview of the core sections of the paper can therefore be given by means of the following table:

\begin{tabular}{c||cccc} 
& $\mathcal{O G}$ & $\mathcal{I G}$ & $\mathcal{A G}$ & $\mathcal{D G}$ \\
\hline \hline $\begin{array}{c}\text { Aristotelian } \\
\text { diagrams }\end{array}$ & Sect. 4 & Sect. 5 & Subsect. 6.1 & Subsect. 6.2 \\
\hline $\begin{array}{c}\text { duality } \\
\text { diagrams }\end{array}$ & Subsect. 7.1 & Subsect. 7.2 & Subsect. 8.1 & Subsect. 8.2
\end{tabular}

This overview table suggests a natural question: why are the opposition and implication relations only used in the diagrams' decorations, and not in the diagram types? In other words, shouldn't it also be possible to study opposition diagrams and implication diagrams for the opposition, implication, Aristotelian and duality relations? The answer is that this is indeed perfectly possible. In Subsection 3.1 it was explained that for every Aristotelian diagram, we can define a corresponding opposition diagram and a corresponding implication diagram (with the original Aristotelian diagram being informationally optimal among the three types of diagrams). For example, given the Aristotelian square for S5 shown in Figure 2(b), we can define the corresponding opposition and implication squares for S5, which are shown in Figure 2(a) and (c), respectively. This account perfectly transfers from object- to metalogical decorations, and hence, for every Aristotelian diagram that has been studied in Sections 4, 5 and 6 of this paper, it is possible to define the corresponding opposition and implication diagrams. However, for reasons of space (and because the Aristotelian diagrams are more informative than their corresponding opposition and implication diagrams anyway), we have focused on the Aristotelian diagrams.

To conclude, we will highlight some general themes that have shown up at various places throughout the paper. The first such consideration is that despite their more abstract nature, metalogical decorations of logical diagrams give rise to the same kinds of linguistic lexicalization patterns as object-logical decorations. For example, in Subsection 4.2 we compared the non-lexicalization of the metalogical notions of 'non-tautology' and 'noncontingency' (Figure 11) to the non-lexicalization of the quantifiers nall and allno. Furthermore, in Subsection 4.3 we pointed out that the strong and weak notions of contrariety are analogous to the bilateral and unilateral readings

\footnotetext{
${ }^{49}$ If a logical diagram is viewed as a graph [34], its type and decoration correspond to the graph's edges and vertices, respectively.
} 
of quantifiers such as some and many (see Figure 13). Observations such as these further reinforce Seuren's claim that "universal lexicalization phenomena are also present in the terminology of the metalogic of logical relations. We surely have not heard the last of this" [72, p. 11].

A second remark concerns the relative importance of non-contradiction $(N C D)$ and non-implication $(N I)$. In [79] these are shown to be the least informative relations of their respective geometries, i.e. $N C D$ is the least informative opposition relation, and $N I$ is the least informative implication relation. From this perspective, these relations seem to be mathematical artefactsneeded solely to turn $\mathcal{O G}$ and $\mathcal{I} \mathcal{G}$ into partitions - without much independent significance. In this paper, by contrast, $N C D$ and $N I$ have played an important role at various places. ${ }^{50}$ For example, in Subsection 4.2 it was shown that $N C D \cap \Delta=\{(\varphi, \varphi) \mid N C D(\varphi, \varphi)\}$ corresponds to the largest class of formulas, viz. the contingencies. This allowed us to define the strong JSB hexagon in Figure 11(b), instead of merely the square in Figure 12. Furthermore, in Subsection 4.5 we argued that the errors in Béziau's hexagon in Figure $17(\mathrm{a}-\mathrm{b})$ are the result of illicitly ignoring $N C D$, and showed how these errors can be corrected by taking this relation into account (see Figure 18). Similarly, in Subsection 5.2 we explained the difference between the strong JSB hexagon for total orders in Figure 21(a) and the RDH for partial orders in Figure 22 in terms of $N I$ (or rather, its abstract analogue, the incomparability relation \#). Finally, as was discussed in Subsection 6.1, NCD and NI can be used to define the notion of unconnectedness or independence (viz. as $N C D \cap N I$ ). In ongoing work [26], we are exploring the interplay between unconnectedness and other notions of logical (in)dependence, which gives rise to the same kind of metalogical diagrams as discussed in this paper.

A third topic of interest is the distinction between 'classical' and 'degenerated' Aristotelian squares. It is well-known that the four categorical statements of syllogistics constitute a 'classical' Aristotelian square. However, it is equally well-known that if we move from syllogistics to contemporary first-order logic, by dropping the assumption of existential import, only the two contradictions are left, and hence the classical Aristotelian square degenerates into a mere Aristotelian cross [30, Section 4]. As we have seen throughout this paper, similar phenomena also occur for metalogical instead of object-logical decorations. For example, in Subsection 4.2 we defined an Aristotelian square for metalogical notions such as tautology and satisfiability, which is shown in Figure 12(a). However, if we drop the assumption that

\footnotetext{
${ }^{50}$ For yet another metalogical illustration of the importance of $N C D$, recall that in 2003 , Béziau [1] famously argued that the Aristotelian relations $C D, C$ and $S C$ are analogous to classical, paracomplete and paraconsistent negation, respectively. However, in more recent work, Béziau has also considered so-called paranormal negations $[2,3,7]$. These are characterized by the fact that a proposition and its paranormal negation can be true together, and can also be false together. But this means exactly that paranormal negation is analogous to $N C D$. Béziau's original analogy can thus be completed as follows: the opposition relations $C D, C, S C$ and $N C D$ are analogous to classical, paracomplete, paraconsistent and paranormal negation, respectively.
} 
the underlying logical system is consistent, then this square degenerates into a cross, as shown in Figure 12(b). Furthermore, in Subsection 6.1 we showed that Löbner's [52, p. 55] four relations yield an Aristotelian square, which is shown in Figure 24(b). However, if we drop the assumption that the first argument of Löbner's relations is satisfiable, then this square also degenerates into a cross, as shown in Figure 24(a). Finally, in Subsection 6.2 we studied an Aristotelian square for the duality relations INEG and ENEG, which is shown in Figure 28(b). However, if we drop the assumption that operators cannot be self-dual, then this square, once more, degenerates into a cross, as shown in Figure 28(a).

A fourth and final recurrent theme is the subtle relation between the different types of diagrams. We have seen that several sets of relations can be used to decorate Aristotelian as well as duality diagrams. In some cases, however, the Aristotelian diagram turns out to depend on certain additional assumptions, whereas the corresponding duality diagram does not. For example, in Subsection 8.1 we studied the duality square for Löbner's relations, which is shown in Figure 32(a). In Subsection 6.1, however, we showed that these relations can also be used to define an Aristotelian square, but whether this square is classical or degenerated, depends on whether the relations' first argument is assumed to be satisfiable; compare Figure 24(a) and (b). Similarly, the six relations in the duality diagram shown in Figure 33(a) can also be used to define an Aristotelian hexagon, but whether this is a JSB or a U4 hexagon again depends on whether the relations' first argument is assumed to be satisfiable; compare Figure 25(a) and (b).

This sharp contrast between Aristotelian and duality diagrams can be seen as the metalogical manifestation of a more general phenomenon that is well-understood at the object-logical level: the Aristotelian relations are sensitive to the deductive power of the underlying logical system, but the duality relations are entirely insensitive to this [23]. ${ }^{51}$ Consider, for example, the formulas $\square p$ and $\square \neg p$ from modal logic. The Aristotelian relation holding between these formulas heavily depends on the particular modal system that we happen to be working in. For example, (i) in the system D, these formulas are contrary, (ii) in the system $\mathrm{D}+\{\diamond p \rightarrow \square p\}$, they are contradictory, and (iii) in the minimal normal system $\mathrm{K}$, they are unconnected, i.e. they do not stand in any Aristotelian relation at all [17]. However, switching from Aristotelian to duality relations, note that in each of these three modal systems, it holds that $\square p$ and $\square \neg p$ are each other's internal negation, which shows that the duality relation holding between these formulas is independent of any additional factors. This contrast between 'context-sensitive' Aristotelian relations and 'context-insensitive' duality relations manifests itself at the metalogical as well as at the object-logical level.

All these remarks and observations point in the direction of a final, overarching conclusion: there exists a fundamental continuity between object- and metalogical decorations of logical diagrams. The mathematical background

${ }^{51}$ Also see Section 2, in particular, the motivation for going from Definition 2.1 to 2.2 . 
of this continuity was described in Section 2 (for Aristotelian diagrams) and in Subsection 3.2 (for duality diagrams). Furthermore, since metalogical decorations give rise to the same kinds of rich logical and linguistic behavior as object-logical decorations, it is to be expected that over time, the former will come to be as widely studied as the latter.

\section{References}

[1] Béziau, J.-Y.: New Light on the Square of Oppositions and its Nameless Corner. Logical Investigations 10, 218-232 (2003)

[2] Béziau, J.-Y.: Bivalent Semantics for De Morgan Logic (The Uselessness of FourValuedness). In: Carnielli, W., Coniglio, M., D'Ottaviano, I. (eds.) The Many Sides of Logic, pp. 391-402. College Publications, London (2009)

[3] Béziau, J.-Y.: Paralogics and the Theory of Valuation. In: Carnielli, W., Coniglio, M., D'Ottaviano, I. (eds.) Universal Logic: An Anthology - From Paul Hertz to Dov Gabbay, pp. 361-372. Springer, Basel (2012)

[4] Béziau, J.-Y.: The Power of the Hexagon. Log. Univers. 6, 1-43 (2012)

[5] Béziau, J.-Y.: The Metalogical Hexagon of Opposition. Argumentos 5, 111-122 (2013)

[6] Béziau, J.-Y.: Opposition and Order. In: Béziau J.-Y., Gan-Krzywoszynska, K. (eds.) New Dimensions of the Square of Opposition, pp. 321-336. Philosophia Verlag, Munich (2014)

[7] Béziau, J.-Y.: Paraconsistent Logic and Contradictory Viewpoints. Revista Brasileira de Filosofia (forthcoming)

[8] Béziau, J.-Y., Jacquette, D. (eds.) Around and Beyond the Square of Opposition. Springer, Basel (2012)

[9] Béziau, J.-Y., Payette, G. (eds.) The Square of Opposition. A General Framework for Cognition. Peter Lang, Bern (2012)

[10] Blanché, R.: Quantity, Modality, and Other Kindred Systems of Categories. Mind 61, 369-375 (1952)

[11] Blanché, R.: Sur l'Opposition des Concepts. Theoria 19, 89-130 (1953)

[12] Blanché, R.: Opposition et Négation. Revue Philosophique de France et de l'Étranger 147, 187-216 (1957)

[13] Blanché, R.: Structures Intellectuelles: Essai sur l'Organisation Systématique des Concepts. Vrin, Paris (1966)

[14] Bochenski, J.: A Precis of Mathematical Logic. Reidel, Dordrecht (1959)

[15] Chatti, S.: Logical Oppositions in Arabic logic: Avicenna and Averroes. In: Béziau J.-Y., Jacquette, D. (eds.) Around and Beyond the Square of Opposition, pp. 21-40. Springer, Basel (2012)

[16] Chatti, S.: Avicenna on Possibility and Necessity. History and Philosophy of Logic 35, 332-353 (2014)

[17] Chellas, B.: Modal Logic. An Introduction. Cambridge University Press, Cambridge (1980)

[18] Copenhaver, B., Normore, C., Parsons, T.: Peter of Spain: Summaries of Logic. Text, Translation, Introduction, and Notes. Oxford University Press, Oxford (2014) 
[19] Czeżowski, T.: On Certain Peculiarities of Singular Propositions. Mind 64, 392-395 (1955)

[20] D'Alfonso, D.: The Square of Opposition and Generalized Quantifiers. In: Béziau J.-Y., Jacquette, D. (eds.) Around and Beyond the Square of Opposition, pp. 219-227. Springer, Basel (2012)

[21] Demey, L.: Algebraic Aspects of Duality Diagrams. In: Cox, P., Plimmer, B., Rodgers, P. (eds.) Diagrammatic Representation and Inference, LNCS 7352, pp. 300-302. Springer, Berlin (2012)

[22] Demey, L.: Structures of Oppositions in Public Announcement Logic. In: Béziau J.-Y., Jacquette, D. (eds.) Around and Beyond the Square of Opposition, pp. 313-339. Springer, Basel (2012)

[23] Demey, L.: Interactively Illustrating the Context-Sensitivity of Aristotelian Diagrams. In: Christiansen, H., Stojanovic, I., Papadopoulos, G. (eds.) Modeling and Using Context, LNCS 9405, pp. 331-345. Springer, Berlin (2015)

[24] Demey, L.: Using Syllogistics to Teach Metalogic. Submitted, 13pp. (2015)

[25] Demey, L.: Partitioning Logical Space. Manuscript (2015)

[26] Demey, L.: Dependence and Independence in Logical Geometry. Manuscript (2016)

[27] Demey, L., Smessaert, H.: Logische geometrie en pragmatiek. In: Van de Velde, F., Smessaert, H., Van Eynde, F., Verbrugge, S. (eds.) Patroon en argument, pp. 553-564. Leuven University Press, Leuven (2014)

[28] Demey, L., Smessaert, H.: The Relationship between Aristotelian and Hasse Diagrams. In: Dwyer, T., Purchase, H., Delaney, A. (eds.) Diagrammatic Representation and Inference, LNCS 8578, pp. 213-227. Springer, Berlin (2014)

[29] Demey, L., Smessaert, H.: Duality in Logic and Language. In: Béziau, J.-Y. (ed.) Encyclopedia of Logic. College Publications, London (2015)

[30] Demey, L., Smessaert, H.: Combinatorial Bitstring Semantics for Arbitrary Logical Fragments. Submitted, 40pp. (2015)

[31] Demey, L., Smessaert, H.: Generating the Logical Relations. Manuscript (2015)

[32] Demey, L., Smessaert, H.: The Logical Geometry of the Aristotelian Rhombic Dodecahedron. Manuscript (2015)

[33] Diaconescu, R.: The Algebra of Opposition (and Universal Logic Interpretations). In: Koslow, A., Buchsbaum, A. (eds.) The Road to Universal Logic, pp. 127-143. Springer, Basel (2015)

[34] Diestel, R.: Graph Theory, 4th Edition. Springer, Berlin (2010)

[35] Fitting, M., Mendelsohn, R.: First-Order Modal Logic. Kluwer, Dordrecht (1998)

[36] Gottschalk, W. H..: The Theory of Quaternality. Journal of Symbolic Logic 18, 193-196 (1953)

[37] Hacker, E.: The Octagon of Opposition. Notre Dame Journal of Formal Logic 16, 352-353 (1975)

[38] Horn, L.: A Natural History of Negation. University of Chicago Press, Chicago, IL (1989)

[39] Horn, L.: Histoire d'*O: Lexical Pragmatics and the Geometry of Opposition. In Béziau, J.-Y., Payette, G. (eds.) The Square of Opposition. A General Framework for Cognition, pp. 393-426. Peter Lang, Bern (2012) 
[40] Hughes, G.: The Modal Logic of John Buridan. In: Corsi, G., Mangione, C., Mugnai, M. (eds.) Atti del convegno internazionale di storia della logica, le teorie delle modalità, pp. 93-11. CLUEB, Bologna (1987)

[41] Humberstone, L.: The Connectives. MIT Press, Cambridge, MA (2011)

[42] Humberstone, L.: Logical Relations. Philosophical Perspectives 27, 175-230 (2013)

[43] Jacoby, P.:A Triangle of Opposites for Types of Propositions in Aristotelian Logic. The New Scholasticism 24, 32-56 (2012)

[44] Jaspers, D.: Logic and Colour. Logica Univers. 6, 227-248 (2012)

[45] Johnson, W.E.: Logic: Part I. Cambridge University Press, London (1921)

[46] Katzir, R., Singh, R.: Constraints on the Lexicalization of Logical Operators. Linguistics and Philosophy 36, 1-29 (2013)

[47] Keynes, J.N.: Studies and Exercises in Formal Logic. MacMillan, London (1884)

[48] Khomskii, Y.: William of Sherwood, singular propositions and the hexagon of opposition. In: Béziau J.-Y., Jacquette, D. (eds.) Around and Beyond the Square of Opposition, pp. 43-60. Springer, Basel (2012)

[49] Kretzmann, N.: William of Sherwood's Introduction to Logic. University of Minnesota Press, Minneapolis (1966)

[50] Lenzen, W.: How to Square Knowledge and Belief. In: Béziau J.-Y., Jacquette, D. (eds.) Around and Beyond the Square of Opposition, pp. 305-311. Springer, Basel (2012)

[51] Libert, T.: Hypercubes of Duality. In: Béziau J.-Y., Jacquette, D. (eds.) Around and Beyond the Square of Opposition, pp. 293-301. Springer, Basel (2012)

[52] Löbner, S.: Quantification as a Major Module of Natural Language Semantics. In: Groenendijk, J., de Jongh, D., Stokhof, M. (eds.) Studies in Discourse Representation Theory and the Theory of Generalized Quantifiers, pp. 53-85. Foris, Dordrecht (1987)

[53] Löbner, S.: Wahr neben Falsch. Duale Operatoren als die Quantoren natürlicher Sprache. Max Niemeyer Verlag, Tübingen (1990)

[54] Löbner, S.: Dual Oppositions in Lexical Meaning. In: Maienborn, C., von Heusinger, K., Portner, P. (eds.) Semantics: An International Handbook of Natural Language Meaning, Volume I, pp. 479506. De Gruyter Mouton, Berlin (2011)

[55] Luzeaux, D., Sallantin, J., Dartnell, C..: Logical Extensions of Aristotle's Square. Log. Univers. 2, 167-187 (2008)

[56] McNamara, J.: In: Zalta, E. N. (ed.) Stanford Encyclopedia of Philosophy (Winter 2014 Edition). CSLI, Stanford (2014)

[57] McCall, S.: Contrariety. Notre Dame Journal of Formal Logic 8, 121-132 (1967)

[58] Mélès, B.: No Group of Opposition for Constructive Logics: The Intuitionistic and Linear Cases. In: Béziau J.-Y., Jacquette, D. (eds.) Around and Beyond the Square of Opposition, pp. 201-217. Springer, Basel (2012)

[59] Moretti, A.: The Geometry of Logical Opposition. PhD thesis, University of Neuchâtel (2009)

[60] Moretti, A.: Why the Logical Hexagon? Log. Univers. 6, 69-107 (2012) 
[61] Moretti, A..: Arrow-Hexagons. In: Koslow, A., Buchsbaum, A. (eds.) The Road to Universal Logic, Volume II, pp. 417-487. Springer, Basel (2015)

[62] Parsons, T.: The Traditional Square of Opposition. In: Zalta, E. N. (ed.) Stanford Encyclopedia of Philosophy (Winter 2014 Edition). CSLI, Stanford (2012)

[63] Peckhaus, V..: Algebra of Logic, Quantification Theory, and the Square of Opposition. In: Béziau J.-Y., Payette, G. (eds.) The Square of Opposition. A General Framework for Cognition, pp. 25-41. Peter Lang, Bern (2012)

[64] Pellissier, R.: "Setting" n-Opposition. Log. Univers. 2, 235-263 (2008)

[65] Peters, S., Westerståhl, D.: Quantifiers in Language and Logic. Oxford University Press, Oxford (2006)

[66] Piaget, J.: Traité de Logique. Essai de Logistique Opératoire. Colin/Dunod, Paris (1949)

[67] Read, S..: John Buridan's Theory of Consequence and his Octagons of Opposition. In: Béziau J.-Y., Jacquette, D. (eds.) Around and Beyond the Square of Opposition, pp. 93-110. Springer, Basel (2012)

[68] Rini, A., Cresswell, M.: The World-Time Parallel. Tense and Modality in Logic and Metaphyiscs. Cambridge University Press, Cambridge (2012)

[69] Sauriol, P.: Remarques sur la Théorie de l'Hexagone Logique de Blanché. Dialogue 7, 374-390 (1968)

[70] Sesmat, A.: Logique II. Les Raisonnements. Hermann, Paris (1951)

[71] Seuren, P.: The Logic of Language. Language from Within, Volume II. Oxford University Press, Oxford (2010)

[72] Seuren, P.: The Metalogical Hexagon. Manuscript, 11 pp. (2014)

[73] Seuren, P., Jaspers, D.: Logico-cognitive Structure in the Lexicon. Language 90, 607-643 (2014)

[74] Smessaert, H.: On the 3D Visualisation of Logical Relations. Log. Univers. 3, 303-332 (2009)

[75] Smessaert, H.: Boolean Differences between Two Hexagonal Extensions of the Logical Square of Oppositions. In: Cox, P., Plimmer, B., Rodgers, P. (eds.) Diagrammatic Representation and Inference, LNCS 7352, pp. 193-199. Springer, Berlin (2012)

[76] Smessaert, H.: Duality and Reversibility: Squares versus Crosses; Part 1: The Logical Geometry of Set Inclusion. Paper read at the Workshop on Logical Geometry and N-Opposition Theory, Leuven (2012)

[77] Smessaert, H.: The Classical Aristotelian Hexagon Versus the Modern Duality Hexagon. Log. Univers. 6, 171-199 (2012)

[78] Smessaert, H., Demey, L.: Logical and Geometrical Complementarities between Aristotelian Diagrams. In: Dwyer, T., Purchase, H., Delaney, A. (eds.) Diagrammatic Representation and Inference, LNCS 8578, pp. 246-260. Springer, Berlin (2014)

[79] Smessaert, H., Demey, L.: Logical Geometries and Information in the Square of Oppositions. Journal of Logic, Language and Information 23, 527-565 (2014)

[80] Smessaert, H., Demey, L.: Béziau's Contributions to the Logical Geometry of Modalities and Quantifiers. In: Koslow, A., Buchsbaum, A. (eds.) The Road to Universal Logic, Volume I, pp. 475-494. Springer, Basel (2015) 
[81] Smessaert, H., Demey, L.: La Géométrie Logique du Dodécaèdre Rhombique des Oppositions. In: Chatti, S. (ed.) Le Carré et ses Extensions: Approches Théoriques, Pratiques et Historiques, pp. 127-157. Université de Tunis, Tunis (2015)

[82] Smessaert, H., Demey, L.: The Unreasonable Effectiveness of Bitstrings in Logical Geometry. Submitted, 17pp. (2015)

[83] van Benthem, J.: Linguistic Universals in Logical Semantics. In Zaefferer, D. (ed.) Semantic Universals and Universal Semantics, pp. 17-36. Foris, Berlin (1991)

[84] Westerståhl, D.: Classical vs. Modern Squares of Opposition, and Beyond. In Béziau, J.-Y., Payette, G. (eds.) The Square of Opposition. A General Framework for Cognition, pp. 195-229. Peter Lang, Bern (2012)

[85] Zellweger, S.: Untapped Potential in Peirce's Iconic Notation for the Sixteen Binary Connectives. In: Houser, N., Roberts, D.D., Van Evra, J. (eds.) Studies in the Logic of Charles Peirce, pp. 334-386. Indiana University Press, Bloomington (1997)

\section{Lorenz Demey}

Center for Logic and Analytic Philosophy

KU Leuven

Belgium

e-mail: lorenz.demey@hiw.kuleuven.be

Hans Smessaert

Department of Linguistics

KU Leuven

Belgium

e-mail: hans.smessaert@arts.kuleuven.be 